\title{
Nonarchimedean geometry, tropicalization, and metrics on curves
}

\author{
Matthew Baker, Sam Payne and Joseph Rabinoff
}

\begin{abstract}
We develop a number of general techniques for comparing analytifications and tropicalizations of algebraic varieties. Our basic results include a projection formula for tropical multiplicities and a generalization of the Sturmfels-Tevelev multiplicity formula in tropical elimination theory to the case of a nontrivial valuation. For curves, we explore in detail the relationship between skeletal metrics and lattice lengths on tropicalizations and show that the maps from the analytification of a curve to the tropicalizations of its toric embeddings stabilize to isometries on finite subgraphs. Other applications include generalizations of Speyer's well-spacedness condition and the KatzMarkwig-Markwig results on tropical $j$-invariants.
\end{abstract}

\section{Introduction}

The recent work of Gubler [Gub07a, Gub07b], in addition to earlier work of Bieri-Groves [BG84], Berkovich [Ber90, Ber99, Ber04], and others, has revealed close connections between nonarchimedean analytic spaces (in Berkovich's sense) and tropical geometry. One such connection is given by the second author's theorem that 'analytification is the inverse limit of all tropicalizations' (see Theorem 1.2). This result is purely topological, providing a natural homeomorphism between the nonarchimedean analytification $X^{\text {an }}$ of a quasiprojective variety $X$ and the inverse limit of all 'extended tropicalizations' of $X$ coming from closed immersions of $X$ into quasiprojective toric varieties that meet the dense torus. In this paper, we develop a number of general techniques for comparing finer properties of analytifications and tropicalizations of algebraic varieties and apply these techniques to explore in detail the relationship between the natural metrics on analytifications and tropicalizations of curves. The proofs of our main results rely on the geometry of formal models and initial degenerations as well as Berkovich's theory of nonarchimedean analytic spaces.

Let $K$ be an algebraically closed field that is complete with respect to a nontrivial nonarchimedean valuation val: $K \rightarrow \mathbb{R} \cup\{\infty\}$. Let $X$ be a nonsingular curve defined over $K$. The underlying topological space of $X^{\text {an }}$ can be endowed with a 'polyhedral' structure locally modeled on an

Received 24 October 2014, accepted in final form 25 May 2015.

2010 Mathematics Subject Classification 14T05 (primary) 14G22, 14M25 (secondary).

Keywords: algebraic curves, tropical curves, tropical geometry, faithful tropicalization, extended tropicalization, initial degeneration, tropical multiplicities, nonarchimedean geometry, analytification, Berkovich spaces, skeletons, well-spacedness.

This journal is (C) Foundation Compositio Mathematica 2016. This article is distributed with Open Access under the terms of the Creative Commons Attribution Non-Commercial License, which permits non-commercial reuse, distribution, and reproduction in any medium, provided that the original work is properly cited. For commercial re-use, please contact the Foundation Compositio Mathematica.

The first and second authors were supported in part by NSF Research Grants DMS-0901487 and DMS-1068689, respectively. 


\section{BAKER, S. PAYNE AND J. RABINOFF}

$\mathbb{R}$-tree. The leaves of $X^{\text {an }}$ are the $K$-points, together with the 'type 4 points' in Berkovich's classification (see Section 3.5). The nonleaves are exactly those points that are contained in an embedded open segment, and the space $\mathbf{H}_{\circ}\left(X^{\text {an }}\right)$ of nonleaves carries a canonical metric which, like the polyhedral structure, is defined using semistable models for $X$. Our primary reference for these results is [BPR13]; see also [Ber90, §4], [Thu05], and [Bak08, §5].

Suppose that $X$ is embedded in a toric variety $Y_{\Delta}$ and meets the dense torus $\mathbf{T}$. The tropicalization $\operatorname{Trop}(X \cap \mathbf{T})$ is a 1-dimensional polyhedral complex with no leaves in the real vector space spanned by the lattice of 1-parameter subgroups of $\mathbf{T}$. All edges of $\operatorname{Trop}(X \cap \mathbf{T})$ have slopes that are rational with respect to the lattice of 1-parameter subgroups, so there is a natural metric on $\operatorname{Trop}(X \cap \mathbf{T})$ given locally by lattice length on each edge, and globally by shortest paths. The metric space $\mathbf{H}_{\circ}\left(X^{\text {an }}\right)$ naturally surjects onto $\operatorname{Trop}(X \cap \mathbf{T})$, but this map is far from being an isometry since infinitely many embedded segments in $\mathbf{H}_{\circ}\left(X^{\text {an }}\right)$ are contracted. Furthermore, even when an edge of $\mathbf{H}_{\circ}\left(X^{\text {an }}\right)$ maps homeomorphically onto an edge of $\operatorname{Trop}(X \cap \mathbf{T})$, this homeomorphism need not be an isometry; see Section 2.5. Nevertheless, each embedded subgraph in $\mathbf{H}_{\circ}\left(X^{\text {an }}\right)$ maps isometrically onto its image in all 'sufficiently large' tropicalizations.

Theorem 1.1. Let $\Gamma$ be a finite embedded subgraph in $\mathbf{H}_{\circ}\left(X^{\text {an }}\right)$. Then there is a closed embedding of $X$ into a quasiprojective toric variety such that $X$ meets the dense torus and $\Gamma$ maps isometrically onto its image in $\operatorname{Trop}(X \cap \mathbf{T})$. Furthermore, the set of all such embeddings is stable and hence cofinal in the system of all embeddings of $X$ into quasiprojective toric varieties whose images meet $\mathbf{T}$.

Here if $\iota: X \hookrightarrow Y_{\Delta}$ and $\iota^{\prime}: X \hookrightarrow Y_{\Delta^{\prime}}$ are closed embeddings into quasiprojective toric varieties such that $X$ meets the dense tori $\mathbf{T}$ and $\mathbf{T}^{\prime}$, then we say that $\iota^{\prime}$ dominates $\iota$ and we write $\iota^{\prime} \geqslant \iota$ if there exists an equivariant morphism of toric varieties $\psi: Y_{\Delta^{\prime}} \rightarrow Y_{\Delta}$ such that $\psi \circ \iota^{\prime}=\iota$ (see Section 5.15). In this case we have an induced map $\operatorname{Trop}\left(X \cap \mathbf{T}^{\prime}\right) \rightarrow \operatorname{Trop}(X \cap \mathbf{T})$; the above theorem says in particular that if $\Gamma$ maps isometrically onto its image in $\operatorname{Trop}(X \cap \mathbf{T})$, then the same is true for $\Gamma \rightarrow \operatorname{Trop}\left(X \cap \mathbf{T}^{\prime}\right)$. In other words, the maps from $\mathbf{H}_{\circ}\left(X^{\text {an }}\right)$ to the tropicalizations of toric embeddings of $X$ stabilize to isometries on every finite subgraph.

Both the analytification and the tropicalization constructions described above for subvarieties of tori globalize in natural ways. The analytification functor extends to arbitrary finite-type $K$-schemes (see [Ber90, Chapters 2 and 3] or [Ber93]), and tropicalization extends to closed subvarieties of toric varieties as follows. If $\Delta$ is a fan in $N_{\mathbb{R}}$ and $Y_{\Delta}$ is the associated toric variety, then there is a natural 'partial compactification' $N_{\mathbb{R}}(\Delta)$ of $N_{\mathbb{R}}$ which is, set theoretically, the disjoint union of the tropicalizations of all torus orbits in $Y_{\Delta}$. The topology on $N_{\mathbb{R}}(\Delta)$ is such that the natural map from $Y_{\Delta}(K)$ extends to a continuous, proper, and surjective map trop: $Y_{\Delta}^{\text {an }} \rightarrow N_{\mathbb{R}}(\Delta)$. As in the case where $Y_{\Delta}$ is the torus $\mathbf{T}$, the tropicalization $\operatorname{Trop}(X)$ of a closed subvariety $X$ in $Y_{\Delta}$ is the closure of $\operatorname{trop}(X(K))$ in $N_{\mathbb{R}}(\Delta)$, and the extended tropicalization map extends to a continuous, proper, surjective map from $X^{\text {an }}$ onto $\operatorname{Trop}(X)$. See $[\operatorname{Pay09a}$, Rab12] and Section 4.2 for further details.

Theorem 1.2 (Payne). Let $X$ be an irreducible quasiprojective variety over $K$. Then the inverse limit of the extended tropicalizations $\operatorname{Trop}(\iota(X))$ over all closed immersions $\iota: X \hookrightarrow Y_{\Delta}$ into quasiprojective toric varieties is canonically homeomorphic to the analytification $X^{\text {an }}$.

The inverse limit in Theorem 1.2 can be restricted to those closed immersions $\iota$ whose images meet the dense torus $T_{\iota}$, and then the homeomorphism maps $X^{\text {an }} \backslash X(K)$ homeomorphically onto the inverse limit of the ordinary tropicalizations $\operatorname{Trop}\left(\iota(X) \cap T_{\iota}\right)$. 


\section{NONARCHIMEDEAN GEOMETRY AND TROPICALIZATION}

When $X$ is a curve, our Theorem 1.1 says that the metric structures on $\operatorname{trop}\left(\iota(X) \cap T_{\iota}\right)$ stabilize to a metric on the subset $\mathbf{H}_{\circ}\left(X^{\text {an }}\right)$ of the inverse limit, and the restriction of this homeomorphism is an isometry. In general, each sufficiently small segment $e$ in $\mathbf{H}_{\circ}\left(X^{\text {an }}\right)$ is mapped via an affine linear transformation with integer slope onto a (possibly degenerate) segment $e^{\prime}$ in $\operatorname{Trop}(X)$. We write $m_{\text {rel }}(e)$ for the absolute value of the slope of this map, so if $e$ has length $\ell$, then its image $e^{\prime}$ has lattice length $m_{\mathrm{rel}}(e) \cdot \ell$. In Corollary 5.9, we relate these 'expansion factors' to tropical multiplicities of edges in $\operatorname{Trop}(X)$. The notation is meant to suggest that $m_{\text {rel }}(e)$ may be thought of in this context as the relative multiplicity of $e$ over $e^{\prime}$. By definition, the tropical multiplicity $m_{\text {Trop }}\left(e^{\prime}\right)$ of an edge $e^{\prime}$ in a suitable polyhedral structure on $\operatorname{Trop}(X)$ is the number of irreducible components, counted with multiplicities, in the initial degeneration $\operatorname{in}_{w}(X \cap \mathbf{T})$ for any $w$ in the relative interior of $e^{\prime}$. These tropical multiplicities are fundamental invariants in tropical geometry and play a key role in the balancing formula. See Section 2.1 for a definition of the initial degeneration $\operatorname{in}_{w}(X \cap \mathbf{T})$ and further discussion of tropical multiplicities.

Theorem 1.3. There is a polyhedral structure on $\operatorname{Trop}(X \cap \mathbf{T})$ with the following properties:

(i) For each edge $e^{\prime}$ in $\operatorname{Trop}(X \cap \mathbf{T})$, there are finitely many embedded segments $e_{1}, \ldots, e_{r}$ in $\mathbf{H}_{\circ}\left(X^{\text {an }}\right)$ mapping homeomorphically onto $e^{\prime}$.

(ii) Any embedded segment in the preimage of $e^{\prime}$ that is disjoint from $e_{1} \cup \cdots \cup e_{r}$ is contracted to a point.

(iii) The tropical multiplicity of $e^{\prime}$ is the sum of the corresponding expansion factors:

$$
m_{\text {Trop }}\left(e^{\prime}\right)=m_{\text {rel }}\left(e_{1}\right)+\cdots+m_{\text {rel }}\left(e_{r}\right) .
$$

The properties above are preserved by subdivision, so they hold for any sufficiently fine polyhedral structure on $\operatorname{Trop}(X \cap \mathbf{T})$. See Proposition 5.4 and Corollary 5.9.

The tropical multiplicity formula in the above theorem gives an important connection to nonarchimedean analytic spaces that is not visible from the definitions. The formula shows, for example, that if $e^{\prime}$ is a small segment in $\operatorname{Trop}(X \cap \mathbf{T})$ whose tropical multiplicity is equal to 1 , then there is a unique segment $e$ in $\mathbf{H}_{\circ}\left(X^{\text {an }}\right)$ mapping homeomorphically onto $e^{\prime}$, and the length of $e$ is equal to the tropical length of $e^{\prime}$. It is well known that the skeleton of the analytification of an elliptic curve with bad reduction is a loop of length equal to minus the valuation of the $j$-invariant (see for example [BPR13, Remark 4.24]), so these formulas explain earlier results of Katz, Markwig, and Markwig on tropical $j$-invariants of genus 1 curves in toric surfaces [KMM08, KMM09]. See, for instance, Example 2.8. The following theorem also provides natural generalizations for genus 1 curves in higher-dimensional toric varieties, as well as curves of arbitrary genus.

TheOrem 1.4. Let $\Gamma^{\prime}$ be a finite embedded subgraph of $\operatorname{Trop}(X \cap \mathbf{T})$, and suppose that $\mathrm{in}_{w}(X \cap \mathbf{T})$ is irreducible and generically reduced for every $w$ in $\Gamma^{\prime}$. Then there is a unique embedded subgraph $\Gamma$ in $\mathbf{H}_{\circ}\left(X^{\text {an }}\right)$ mapping homeomorphically onto $\Gamma^{\prime}$, and this homeomorphism is an isometry.

See Section 6 for details on deducing the tropical $j$-invariant results of Katz, Markwig, and Markwig from this theorem.

The expansion factors $m_{\text {rel }}(e)$ in our tropical multiplicity formula are often computable in practice. If $X$ is an affine curve embedded in the torus $\mathbf{G}_{m}^{n}$ via an $n$-tuple of invertible regular functions $f_{1}, \ldots, f_{n}$, then

$$
m_{\mathrm{rel}}(e)=\operatorname{gcd}\left(s_{1}(e), \ldots, s_{n}(e)\right)
$$




\section{BAKer, S. PAYne And J. RABinoff}

where $s_{i}(e)$ is the absolute value of the slope of the integer-affine function $\log \left|f_{i}\right|$ along the edge $e$. See Remark 5.6. The quantities $s_{i}(e)$ are easily calculated from the divisors of $f_{1}, \ldots, f_{n}$ using the 'slope formula' of [BPR13, Theorem 5.15].

In concrete situations, it is useful to be able to certify that a given tropicalization map faithfully represents a large piece of the nonarchimedean analytification $X^{\text {an }}$ (for example, the 'minimal skeleton' $\Sigma$ of $X^{\text {an }}$ in the sense of Berkovich [Ber90] or [BPR13, Corollary 4.23]) using only 'tropical' computations (for example, Gröbner complex computations which have been implemented in computer algebra packages such as Singular or Macaulay2), as opposed to calculations with formal models that have not been implemented in a systematic way in any existing software package. We prove that a tropicalization map represents $\Sigma$ faithfully, meaning that the map is an isometry on $\Sigma$, provided that certain combinatorial and topological conditions are satisfied. Our results on faithful representations are presented in conjunction with some observations about initial degenerations which help explain the special role played by trivalent graphs in the literature on tropical curves (cf. Theorem 5.25 and Remark 5.27).

We explore tropicalizations of elliptic curves in detail as a concrete illustration of our methods and results. We are able to say some rather precise things in this case; for example, we show that every elliptic curve $E / K$ with multiplicative reduction admits a closed embedding in $\mathbb{P}^{2}$ whose tropicalization faithfully (and certifiably) represents the minimal skeleton of $E^{\text {an }}$. Furthermore, we interpret Speyer's 'well-spacedness condition' for trivalent tropicalizations of totally degenerate genus 1 curves [Spe14] as a statement about rational functions on the analytification of the curve, and prove generalizations of this condition for nontrivalent tropicalizations, and for genus 1 curves with good reduction.

The paper concludes with a generalization of the important Sturmfels-Tevelev multiplicity formula, which calculates $\operatorname{Trop}(\alpha(X)$ ) (as a weighted polyhedral complex) in terms of $\operatorname{Trop}(X)$ when $\alpha: \mathbf{T} \rightarrow \mathbf{T}^{\prime}$ is a homomorphism of tori which induces a generically finite map from a subvariety $X$ in $\mathbf{T}$ onto its image. The multiplicity formula in [ST08] is formulated and proved in the 'constant coefficient' setting, where $K$ is the field of Puiseux series over an algebraically closed coefficient field $k$ of characteristic 0 and $X$ is defined over $k$. We use the methods of this paper to generalize the Sturmfels-Tevelev formula to the case where $X$ is any closed subvariety of a torus $\mathbf{T}$ defined over a complete and algebraically closed nonarchimedean field $K$.

Philosophically speaking, there are at least two long-term goals to this paper. On the one hand, we believe that the systematic use of modern tools from nonarchimedean geometry is extremely useful for understanding and proving theorems in tropical geometry. This paper takes several steps in that direction, establishing some new results in tropical geometry via Berkovich's theory and the Bosch-Lütkebohmert-Raynaud theory of admissible formal schemes. On the other hand, much of this paper can be viewed as a comparison between two different ways of approximating nonarchimedean analytic spaces. Nonarchimedean analytic spaces have proved to be useful in many different contexts, but the topological spaces underlying them are wildly branching infinite complexes which are difficult to study directly, so one usually approximates them with finite polyhedral complexes. One such approximation goes through skeleta of nice (for example, semistable) formal models (cf. [BPR13, Theorem 5.2]), another through (extended) tropicalizations (cf. Theorem 1.2). Our Theorem 5.21 shows that, in the case of curves, these two approximations have the same metric structure in the limit (though the metrics may be different at any given finite level).

For further details and examples, we refer the reader to the expanded, earlier preprint version of this paper, arxiv:1104.0320v2. This final version differs from that one in several respects. The 


\section{NONARCHIMEDEAN GEOMETRY AND TROPICALIZATION}

numbering (of equations, paragraphs, sections, theorems, etc.) has changed. The former Section 5, The structure theory of analytic curves, was extracted and published separately [BPR13]. Furthermore, much expository material and many examples in the remaining sections have been omitted. The earlier preprint version remains available on the arXiv.

Since this paper was written, there have been a large number of follow-up articles: the reader may also want to read [GRW14], in which many of the results in this article are extended to higher dimensions; [CHW14, DP14, CS13], in which several interesting examples of faithful tropicalizations are given; and [ABBR15a, ABBR15b], in which 'relative' versions of some of the results in this paper are used to prove tropical lifting theorems.

\section{Basic notions and examples}

Here we give a brief overview of the basic notions necessary to understand the theorems stated in the introduction, followed by a few key examples illustrating these results. Throughout this paper, $K$ is an algebraically closed field that is complete with respect to a nontrivial nonarchimedean valuation

$$
\text { val: } K \rightarrow \mathbb{R} \cup\{\infty\} .
$$

We let $G=\operatorname{val}\left(K^{\times}\right)$be its value group, $R=\operatorname{val}^{-1}([0, \infty])$ its valuation $\operatorname{ring}, \mathfrak{m} \subset R$ the maximal ideal, and $k=R / \mathfrak{m}$ its residue field (which is algebraically closed by [Rob00, $\S 2.1$, Proposition 3$]$ ). Let $|\cdot|=\exp (-\operatorname{val}(\cdot))$ be the absolute value on $K$ associated with the valuation.

\subsection{Tropicalization}

Let $M$ be a free abelian group of rank $n$, let $\mathbf{T}=\operatorname{Spec}(K[M])$ be the $K$-torus with character group $M$, and let $N=\operatorname{Hom}(M, \mathbb{Z})$ be the dual lattice. If $X$ is a closed subscheme of $\mathbf{T}$, there is a natural tropicalization map

$$
\text { trop: } X(K) \rightarrow N_{\mathbb{R}}
$$

where $N_{\mathbb{R}}=\operatorname{Hom}(M, \mathbb{R})$. The image of a point $x$ in $X(K)$ is the linear function taking $u \in M$ to the valuation of the corresponding character evaluated at $x$. Then $\operatorname{Trop}(X)$ is the closure of $\operatorname{trop}(X(K))$ in the Euclidean topology on $N_{\mathbb{R}}$. Note that the choice of an isomorphism $M \cong \mathbb{Z}^{n}$ induces an identification of $\mathbf{T}$ with $\mathbf{G}_{m}^{n}$. In such coordinates, the tropicalization map sends a point $\left(x_{1}, \ldots, x_{n}\right)$ in $X(K)$ to $\left(\operatorname{val}\left(x_{1}\right), \ldots, \operatorname{val}\left(x_{n}\right)\right)$ in $\mathbb{R}^{n}$.

One of the basic results in tropical geometry says that if $X$ is an integral subscheme of $\mathbf{T}$ of dimension $d$, then $\operatorname{Trop}(X)$ is the underlying set of a connected 'balanced weighted integral $G$-affine polyhedral complex' of pure dimension $d$. We do not define all of these terms here, but briefly recall how one gets a polyhedral complex and defines weights on the maximal faces of this complex. Let $w$ be a point in $N_{G}=\operatorname{Hom}(M, G)$. The 'tilted group ring' $R[M]^{w}$ is the subring of $K[M]$ consisting of Laurent polynomials $a_{1} x^{u_{1}}+\cdots+a_{r} x^{u_{r}}$ such that

$$
\operatorname{val}\left(a_{i}\right)+\left\langle u_{i}, w\right\rangle \geqslant 0
$$

for all $i$. The $R$-scheme $\mathbf{T}^{w}=\operatorname{Spec} R[M]^{w}$ is a torsor for the torus Spec $R[M]$, and its generic fiber is canonically isomorphic to $\mathbf{T}$. If $X$ is a closed subscheme of $\mathbf{T}$ defined by an ideal $\mathfrak{a} \subset K[M]$, then

$$
X^{w}=\operatorname{Spec}\left(R[M]^{w} /\left(\mathfrak{a} \cap R[M]^{w}\right)\right)
$$

is a flat $R$-scheme with generic fiber $X$, which we call the tropical integral model associated with $w$. It is exactly the closure of $X$ in $\mathbf{T}^{w}$. The special fiber $\operatorname{in}_{w}(X)$ of $X^{w}$ is called the initial 


\section{BAKer, S. PAYne And J. RABinofF}

degeneration of $X$ with respect to $w$ and is the subscheme of the special fiber of $\mathbf{T}^{w}$ cut out by the $w$-initial forms of Laurent polynomials in $\mathfrak{a}$, in the sense of generalized Gröbner theory.

The scheme $\mathbf{T}^{w}$ is not proper, so points in $X(K)$ may fail to have limits in the special fiber. Indeed, the special fiber $\operatorname{in}_{w}(X)$ is often empty. One of the fundamental theorems in tropical geometry says that $w$ is in $\operatorname{Trop}(X)$ if and only if $\operatorname{in}_{w}(X)$ is not empty. ${ }^{1}$ Moreover, $\operatorname{Trop}(X)$ can be given the structure of a finite polyhedral complex in such a way that whenever $w$ and $w^{\prime}$ belong to the relative interior of the same face, the corresponding initial degenerations $\operatorname{in}_{w}(X)$ and $\operatorname{in}_{w^{\prime}}(X)$ are $\mathbf{T}$-affinely equivalent.

We define the multiplicity $m_{\operatorname{Trop}}(w)$ of a point $w$ in $\operatorname{Trop}(X)$ to be the number of irreducible components of $\operatorname{in}_{w}(X)$, counted with multiplicities. In particular, $m_{\text {Trop }}(w)=1$ if and only if $\operatorname{in}_{w}(X)$ is irreducible and generically reduced. These tropical multiplicities are constant on the relative interior of each face $F$ of $\operatorname{Trop}(X)$, and we define the multiplicity $m_{\text {Trop }}(F)$ to be $m_{\text {Trop }}(w)$ for any $w$ in the relative interior of $F$. The multiplicities for maximal faces are the 'weights' mentioned above that appear in the balancing condition. These weights have the following simple interpretation for hypersurfaces.

Remark 2.2. If $X=V(f)$ is a hypersurface, then $\operatorname{Trop}(X)$ is the corner locus of the convex piecewise-linear function associated with a defining equation $f$ [EKL06, $\S 2.1]$. In this case, $\operatorname{Trop}(X)$ has a unique minimal polyhedral structure, and the initial degenerations are essentially constant on the relative interior of each face. There is a natural inclusion reversing bijection between the faces of $\operatorname{Trop}(X)$ in this minimal polyhedral structure and the positive-dimensional faces of the Newton polytopal complex (or Newton complex) of $f$ : a face of $\operatorname{Trop}(X)$ corresponds to the convex hull of the monomials whose associated affine linear function is minimal on that face. In particular, the maximal faces of $\operatorname{Trop}(X)$ correspond to the edges of this Newton complex. In this special case, the multiplicity of a maximal face is the lattice length of the corresponding edge. ${ }^{2}$ The relationship between the tropical hypersurface and the Newton complex is also explained in more detail in $[$ Rab12, $\S 8]$.

\subsection{Analytification}

Let $A$ be a finite-type $K$-algebra. The Berkovich spectrum of $A$, denoted $\mathscr{M}(A)$, is defined to be the set of multiplicative seminorms $\|\cdot\|$ on $A$ extending the absolute value $|\cdot|$ on $K$. The Berkovich spectrum $\mathscr{M}(A)$ is the underlying set of the nonarchimedean analytification $X^{\text {an }}$ of $X=\operatorname{Spec}(A)$. The topology on $X^{\text {an }}$ is the coarsest such that the map $\|\cdot\| \mapsto\|f\|$ is continuous for every $f \in A$; this coincides with the subspace topology induced by the inclusion of $X^{\text {an }}$ in $\mathbb{R}^{A}$.

Remark. We will often write $\mathbb{A}_{\text {an }}^{1}$ for $\mathbb{A}^{1 \text {,an }}$ and $\mathbb{P}_{\text {an }}^{1}$ for $\mathbb{P}^{1 \text {,an }}$, etc.

If $X$ is connected, then $X^{\text {an }}$ is a path-connected locally compact Hausdorff space that naturally contains $X(K)$ as a dense subset; a point $x \in X(K)$ corresponds to the seminorm $\|\cdot\|_{x}$ given by $\|f\|_{x}=|f(x)|$. The analytification procedure $X \mapsto X^{\text {an }}$ gives a covariant functor from the category of locally finite-type $K$-schemes to the category of topological spaces. ${ }^{3}$

\footnotetext{
${ }^{1}$ In the special case where $\mathbf{T}$ has dimension 1 and $X$ is the zero locus of a Laurent polynomial $f$, this is equivalent to the statement that $f$ has a root with valuation $s$ if and only if $-s$ is a slope of the Newton polygon of $f$.

${ }^{2}$ This is explained in [ST08, Example 3.16] in the special case where $X$ is irreducible, $K$ is the field of Puiseux series over $k$, and $X$ is defined over $k$. The arguments given there work in full generality.

${ }^{3}$ The analytification $X^{\text {an }}$ has additional structure, including a structure sheaf, and $X \mapsto X^{\text {an }}$ may also be seen as a functor from locally finite-type $K$-schemes to locally ringed spaces. See [Ber90, $\S \S 2.3,3.1,3.4]$ for more details.
} 


\section{NONARCHIMEDEAN GEOMETRY AND TROPICALIZATION}

If $X$ is a closed subvariety of $\mathbf{T}$, then the tropicalization map described in the previous section extends from $X(K)$ to a continuous and proper map

$$
\text { trop: } X^{\text {an }} \rightarrow N_{\mathbb{R}}
$$

taking a seminorm $\|\cdot\|$ to the linear function $u \mapsto-\log \left\|x^{u}\right\|$, and the image of this map is exactly $\operatorname{Trop}(X)$. In other words, $\operatorname{trop}\left(X^{\text {an }}\right)$ is the closure of the image of $X(K)$ in $N_{\mathbb{R}}$.

\subsection{Metric structure of analytic curves}

There is a natural metric on $\mathbb{P}_{\text {an }}^{1} \backslash \mathbb{P}^{1}(K)$; see [BPR13] for details. We write $\mathbf{H}\left(\mathbb{P}_{\text {an }}^{1}\right)$ to denote $\mathbb{P}_{\text {an }}^{1} \backslash \mathbb{P}^{1}(K)$ with this metric structure. It is important to note that the metric topology on $\mathbf{H}\left(\mathbb{P}_{\text {an }}^{1}\right)$ is much finer than the subspace topology on $\mathbb{P}_{\text {an }}^{1} \backslash \mathbb{P}^{1}(K)$. Our notation follows [BR10] and reflects the fact that the metric on $\mathbf{H}\left(\mathbb{P}_{\text {an }}^{1}\right)$ is 0-hyperbolic in the sense of Gromov.

The metric on $\mathbf{H}\left(\mathbb{P}_{\text {an }}^{1}\right)$ has the important property that, roughly speaking, $\log |f|$ is piecewise affine with integer slopes for any nonzero rational function $f \in K(T)$. More precisely, suppose that $f$ is nonconstant and that $\widehat{\Sigma}$ is the minimal closed connected subset of $\mathbb{P}_{\text {an }}^{1}$ containing the set $S$ of zeros and poles of $f$. Let $\Sigma=\widehat{\Sigma} \backslash S$. Then:

(i) The subspace $\Sigma$ of $\mathbf{H}\left(\mathbb{P}_{\text {an }}^{1}\right)$ is a metric graph with finitely many edges, in which the edges whose closures meet $K$ have infinite length.

(ii) The restriction of $\log |f|$ to $\Sigma$ is piecewise affine with integer slopes.

(iii) There is a natural retraction map from $\mathbb{P}_{\text {an }}^{1}$ onto $\widehat{\Sigma}$.

(iv) The function $\log |f|$ from $\mathbb{P}_{\text {an }}^{1}$ to $\mathbb{R} \cup\{ \pm \infty\}$ factors through the retraction onto $\widehat{\Sigma}$, and hence is determined by its restriction to $\Sigma$.

The metric on the complement of the set of $K$-points in the analytification of an arbitrary algebraic curve is induced by the metric on $\mathbb{P}_{\text {an }}^{1} \backslash \mathbb{P}^{1}(K)$ via semistable decomposition; see [BPR13] for details.

There is also a notion of a skeleton of a smooth and connected but not necessarily complete curve $X$. Let $\widehat{X}$ be the smooth compactification of $X$, and let $D=\widehat{X} \backslash X$ be the set of 'punctures'. Choose a semistable model $\mathcal{X}$ of $\widehat{X}$ such that the punctures reduce to distinct smooth points of the special fiber $\overline{\mathcal{X}}$. Then there is unique minimal closed connected subset $\Sigma$ of $X^{\text {an }}$ which contains the skeleton $\Sigma_{\mathcal{X}}$ of $\widehat{X}$ and whose closure in $\widehat{X}^{\text {an }}$ contains $D$. We call $\Sigma$ the skeleton of $X$ associated with $\mathcal{X}$. As above, there is a canonical retraction map $\tau_{\Sigma}: X^{\text {an }} \rightarrow \Sigma$. If $X \subset \mathbf{T}$, then the tropicalization map trop: $X \rightarrow N_{\mathbb{R}}$ factors through $\tau_{\Sigma}$. There is a skeleton which is minimal over all models $\mathcal{X}$ if $2-2 g(\widehat{X})-\# D \leqslant 0$. See [BPR13] for a complete discussion of the skeleta of a curve.

\subsection{Examples}

To illustrate our main results concerning the relationship between analytification and tropicalization in the case of curves, taking into account the metric structure on both sides, we present the following examples. In each example, we fix a specific coefficient field for concreteness.

Our first example shows how a loop in the analytification of a genus 1 curve can be collapsed onto a segment of multiplicity greater than 1 .

EXAmple 2.6. Let $K$ be the completion of the field of Puiseux series $\mathbb{C}\{\{t\}\}$. Consider the genus 1 curve $\widehat{E} \subset \mathbb{P}^{2}$ over $K$ defined by the Weierstrass equation $y^{2}=x^{3}+x^{2}+t^{4}$, and let $E=\widehat{E} \cap \mathbf{G}_{m}^{2}$. The $j$-invariant of $\widehat{E}$ has valuation -4 , so $\widehat{E}$ has multiplicative reduction and the minimal skeleton 


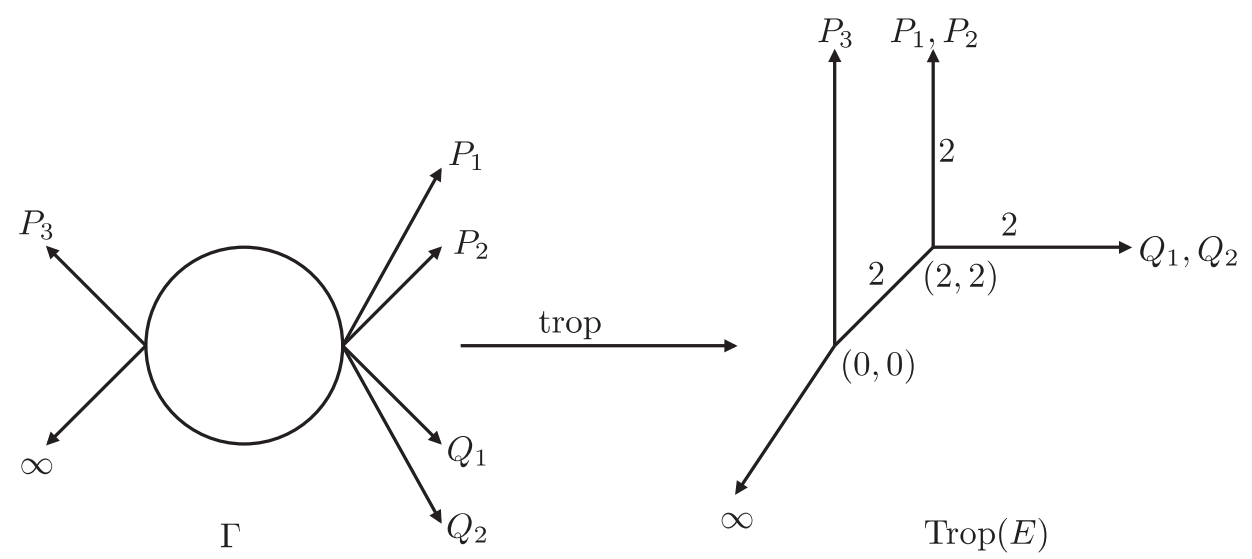

Figure 1. The minimal skeleton $\Gamma \subset E^{\text {an }}$ and the tropicalization $\operatorname{Trop}(E)$ from Example 2.6. The points $P_{i}, Q_{j}$ are defined as follows: the rational function $x$ on $\widehat{E}$ has divisor $\left(Q_{1}\right)+\left(Q_{2}\right)-2(\infty)$, where $Q_{1}=\left(0, t^{2}\right)$ and $Q_{2}=\left(0,-t^{2}\right)$, and $y$ has divisor $\left(P_{1}\right)+\left(P_{2}\right)+\left(P_{3}\right)-3(\infty)$, where $\operatorname{val}\left(x\left(P_{1}\right)\right)=\operatorname{val}\left(x\left(P_{2}\right)\right)=2$ and $\operatorname{val}\left(x\left(P_{3}\right)\right)=0$.

$\Sigma$ of $\widehat{E}$ is isometric to a circle of circumference 4 . In this example, $\operatorname{Trop}(E)$ does not have a cycle even though $\widehat{E}^{\text {an }}$ does; it is interesting to examine exactly what tropicalization is doing to $\widehat{E}^{\text {an }}$. Let $\Gamma$ be the minimal skeleton of $E$; as above, trop factors through the retraction of $E^{\text {an }}$ onto $\Gamma$. Figure 1 shows the restriction of trop to $\Gamma$.

The tropicalization map sends $\Sigma$ two-to-one onto its image in $\operatorname{Trop}(E)$, which is a segment of tropical length 2 and tropical multiplicity 2 . Locally on $\Sigma$ the tropicalization map is an isometry. Each of the rays of $\Gamma$ emanating from $\Sigma$ maps isometrically onto its image. The two rays in $\operatorname{Trop}(E)$ with multiplicity 1 have unique preimages in $\Gamma$, while there are two distinct rays in $\Gamma$ mapping onto each of the two rays in $\operatorname{Trop}(E)$ of multiplicity 2.

The following example illustrates a different kind of collapse, where a segment $e$ in the minimal skeleton of the analytification is collapsed to a point, that is, the relative multiplicty $m_{\text {rel }}(e)$ is 0 .

EXAMPLE 2.7. Let $p \geqslant 5$ be a prime, let $K=\mathbb{C}_{p}$, and let $k \cong \overline{\mathbf{F}}_{p}$ be its residue field. Let $X \subset \mathbf{G}_{m}^{2}$ be the affine curve over $K$ defined by the equation $f(x, y)=x^{3} y-x^{2} y^{2}-2 x y^{3}-3 x^{2} y+2 x y-p=0$. The curve $\widehat{X} \subset \mathbb{P}^{2}$ defined by the homogenization $\widehat{f}(x, y, z)=x^{3} y-x^{2} y^{2}-2 x y^{3}-3 x^{2} y z+2 x y z^{2}-$ $p z^{4}=0$ is a smooth plane quartic of genus 3 , and the given equation $\widehat{f}(x, y, z)=0$ defines the minimal regular proper semistable model $\mathcal{X}$ for $\widehat{X}$ over $\mathbb{Q}_{p}$. The special fiber $\overline{\mathcal{X}}$ of $\mathcal{X}$ consists of four (reduced) lines in general position in $\mathbb{P}_{k}^{2}$, since $\widehat{f} \bmod p$ factors as $x y(x+y-z)(x-2 y-2 z)$. The tropicalization $\operatorname{Trop}(X) \subset \mathbb{R}^{2}$ consists of a triangle with vertices $(0,0),(1,0),(0,1)$ together with three rays emanating from these three vertices in the directions of $(-1,-1),(3,-1),(-1,3)$ respectively. The three bounded edges/rays incident to $(0,0)$ all have tropical multiplicity 2 , and all other bounded edges/rays in $\operatorname{Trop}(X)$ have tropical multiplicity 1 . Let $\Sigma$ be the skeleton $\Sigma_{\mathcal{X}}$ of $\widehat{X}$, and let $\Gamma$ be the minimal skeleton of $X$. Then $\Sigma$ is a tetrahedron (with vertices corresponding to the four irreducible components of $\overline{\mathcal{X}}$ ) with six edges of length 1 , because this is the dual graph of a regular semistable model defined over $\mathbb{Z}_{p}$, and $\Gamma$ is obtained from $\Sigma$ by adding a ray emanating from each vertex of $\Sigma$ toward the zeros and poles of $x$ and $y$, namely toward the points $(0: 1: 0)$, $(1: 0: 0),(2: 1: 0),(-1: 1: 0)$. The tropicalization map trop: $X^{\text {an }} \rightarrow \operatorname{Trop}(X) \subset \mathbb{R}^{2}$ factors though the retraction map $X^{\text {an }} \rightarrow \Gamma$. See Figure 2 .

The points of $\Sigma$ corresponding to the irreducible components $x+y=z$ and $x-2 y=2 z$ of $\overline{\mathcal{X}}$, 


\section{NONARCHIMEDEAN GEOMETRY AND TROPICALIZATION}

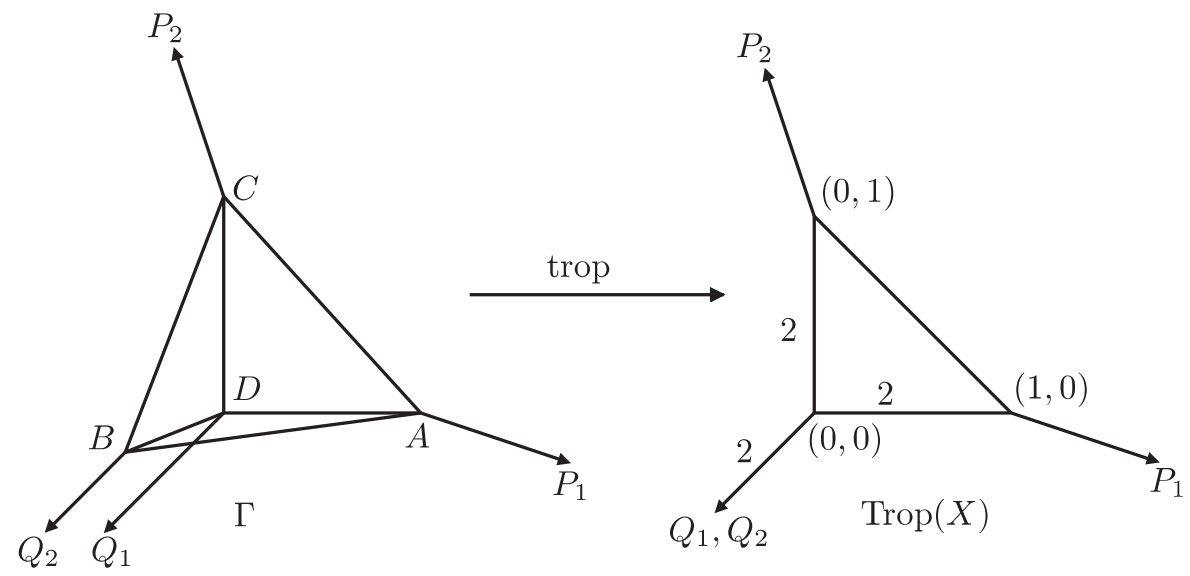

Figure 2. The skeleton $\Gamma \subset X^{\text {an }}$ and the tropicalization $\operatorname{Trop}(X)$, where $X$ is the curve from Example 2.7. Here $x$ has divisor $3\left(P_{1}\right)-\left(P_{2}\right)-\left(Q_{1}\right)-\left(Q_{2}\right)$ and $y$ has divisor $3\left(P_{2}\right)-\left(P_{1}\right)-$ $\left(Q_{1}\right)-\left(Q_{2}\right)$ on $\widehat{X}$, where $P_{1}=(0: 1: 0), P_{2}=(1: 0: 0), Q_{1}=(2: 1: 0)$, and $Q_{2}=(-1: 1: 0)$. The points $A, B, C, D \in \Gamma$ correspond to the irreducible components $x=0, x+y=z, y=0$, and $x-2 y=2 z$, respectively, of $\overline{\mathcal{X}}$. The collapsed segment is $\overline{B D}$.

as well as the entire edge of $\Sigma$ connecting these two points, get mapped by trop to the point $(0,0) \in \operatorname{Trop}(X)$. This edge therefore has expansion factor 0 with respect to trop. The other five bounded edges of $\Sigma \subset \Gamma$ map isometrically (that is, with expansion factor 1) onto their images in $\operatorname{Trop}(X)$. In fact, the tropicalization map is a local isometry everywhere on $\Gamma$ except along the bounded edge which is contracted to the origin.

Our final example, which is meant to illustrate Theorem 1.4, is a genus 1 curve with multiplicative reduction for which the tropicalization map takes the minimal skeleton isometrically onto its image. This example is also discussed in [KMM09, Example 5.2].

Example 2.8. Let $K$ be the completion of the field $\mathbb{C}\{\{t\}\}$ of Puiseux series. Consider the curve $E^{\prime}$ in $\mathbf{G}_{m}^{2}$ cut out by the equation $f(x, y)=x^{2} y+x y^{2}+\frac{1}{t} x y+x+y$. Its closure in $\mathbb{P}^{2}$ is the smooth projective genus 1 curve $\widehat{E}^{\prime}$ defined by $\widehat{f}(x, y, z)=x^{2} y+x y^{2}+\frac{1}{t} x y z+x z^{2}+y z^{2}$.

Using the description of $\operatorname{Trop}\left(E^{\prime}\right)$ as the corner locus of the convex piecewise-linear function associated with $f$, one sees that $\operatorname{Trop}\left(E^{\prime}\right)$ consists of a square with side length 2 plus one ray emanating from each corner of the square; see Figure 3. By restricting $f$ to faces of the Newton complex (see Remark 2.2), one checks that $\operatorname{in}_{w}\left(E^{\prime}\right)$ is reduced and irreducible for every $w$ in $\operatorname{Trop}\left(E^{\prime}\right)$. Therefore, by Theorem 1.4 there is a unique graph $\Gamma$ in the analytification of $E^{\prime}$ mapping isometrically onto $\operatorname{Trop}(X)$.

In particular, the analytification of $E^{\prime}$ contains a loop of length 8 . One can check by an explicit computation that $\operatorname{val}\left(j\left(\widehat{E}^{\prime}\right)\right)=-8$, which is consistent with the fact that the analytification of a smooth projective genus 1 curve is either contractible (if the curve has good reduction) or else contains a unique loop of length $-\operatorname{val}(j)$ (if the curve has multiplicative reduction).

\section{Admissible algebras and nonarchimedean analytic spaces}

Recall that $K$ is an algebraically closed field that is complete with respect to a nontrivial nonarchimedean valuation val: $K \rightarrow \mathbb{R} \cup\{\infty\}$. Let $|\cdot|=\exp (-\operatorname{val}(\cdot))$ be the associated absolute value. 


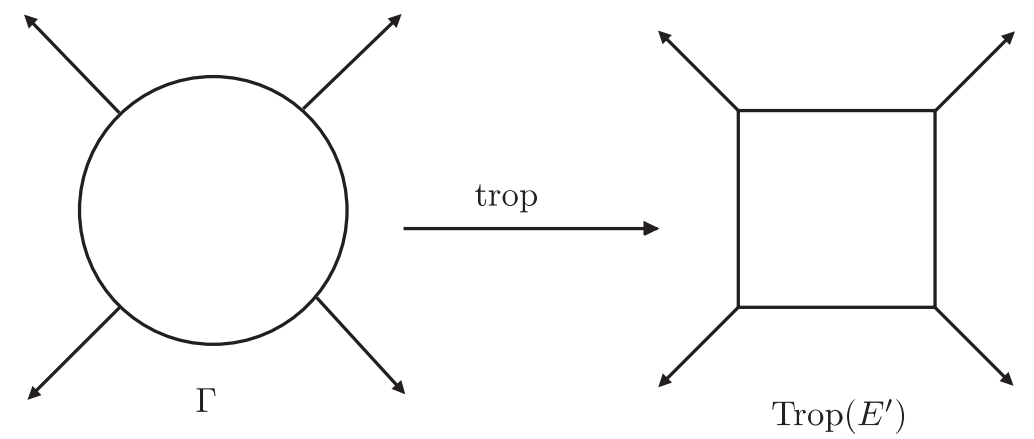

Figure 3. The tropicalization of the elliptic curve $\widehat{E}^{\prime}$ from Example 2.8. The edges in the square each have lattice length 2.

Let $R$ be the valuation ring of $K$, let $\mathfrak{m} \subset R$ be its maximal ideal, and let $k=R / \mathfrak{m}$ be its residue field. Choose a nonzero element $\varpi \in \mathfrak{m}$ (as $R$ is not noetherian, it has no uniformizer), so $R$ is $\varpi$-adically complete. Let $G=\operatorname{val}\left(K^{\times}\right) \subset \mathbb{R}$ be the value group, which is divisible.

In this section we define some notation and collect some results about admissible $R$-algebras and Raynaud's generic fiber functor that will be needed in the sequel. We refer the reader to [BL93] and [Bos14] for a detailed discussion of admissible formal schemes and the Raynaud generic fiber functor.

\subsection{Admissible formal schemes}

An admissible $R$-algebra is a topological $R$-algebra $A$ which is flat and topologically of finite presentation. A formal scheme $\mathfrak{X}$ over $\operatorname{Spf}(R)$ which is locally isomorphic to the formal spectrum of an admissible $R$-algebra (in its $\varpi$-adic topology) is called an admissible formal scheme. All admissible formal schemes appearing in this paper will be assumed to be quasi-compact and separated. If $\mathfrak{X}=\operatorname{Spf}(A)$, then we write $\mathfrak{X}^{\text {an }}$ for the $K$-affinoid Berkovich analytic space $\mathscr{M}\left(A \otimes_{R} K\right)$. This construction globalizes to give a functor from admissible formal schemes to Berkovich analytic spaces that is compatible with fiber products. The image of a formal scheme under this functor is called the Raynaud generic fiber.

An admissible formal scheme $\mathfrak{X}$ with reduced special fiber is called a formal analytic variety. If $\operatorname{Spf}(A)$ is a formal affine open subset of a formal analytic variety, then $A$ is reduced and $A$ is equal to the full ring of power-bounded elements in $A_{K}:=A \otimes_{R} K$ by Proposition 3.13. The canonical reduction $\widetilde{A}_{K}$ of $A_{K}$ therefore coincides with $A \otimes_{R} k$, so if $\operatorname{Spf}(B)$ is a formal affine open subset of $\operatorname{Spf}(A)$, then $\operatorname{Spec}\left(\widetilde{B}_{K}\right)$ is an affine open subset of $\operatorname{Spec}\left(\widetilde{A}_{K}\right)$. (Our definition of a formal analytic variety differs from the original one given in [BL85], but the above argument shows that the two definitions are equivalent in our situation.)

Notation. Let $\mathfrak{X}$ be an $R$-scheme or a formal $R$-scheme. We denote the special fiber $\mathfrak{X} \otimes_{R} k$ of $\mathfrak{X}$ by $\overline{\mathfrak{X}}$.

\subsection{Reductions of analytic spaces}

Let $\mathcal{A}$ be a $K$-affinoid algebra. We denote by $\mathcal{A}, \check{\mathcal{A}}$, and $\widetilde{\mathcal{A}}=\check{\mathcal{A}} / \check{\mathcal{A}}$ the subring of powerbounded elements, the ideal of topologically nilpotent elements, and the canonical reduction, respectively. Setting $\mathscr{X}=\mathscr{M}(\mathcal{A})$, there is a reduction map red: $\mathscr{X} \rightarrow \widetilde{\mathscr{X}}:=\operatorname{Spec}(\widetilde{\mathcal{A}})$. By $[\operatorname{Ber} 90$, Corollary 2.4.2 and Proposition 2.4.4] this map is surjective and anti-continuous, in the sense that 


\section{NONARCHIMEDEAN GEOMETRY AND TROPICALIZATION}

the inverse image of an open subset is closed. Similarly, if $\mathfrak{X}$ is an admissible formal scheme over $\operatorname{Spf}(R)$, then there is a canonical surjective and anti-continuous reduction map red: $\mathfrak{X}^{\text {an }} \rightarrow \overline{\mathfrak{X}}$ which coincides with the map defined above when $\mathfrak{X}=\operatorname{Spf}(\mathcal{A})$. In particular, $\overline{\mathfrak{X}}$ is connected if $\mathfrak{X}^{\text {an }}$ is connected. The inverse image of a closed point of $\overline{\mathfrak{X}}$ under the reduction map is called a formal fiber.

\subsection{Shilov points}

The Shilov boundary of a $K$-affinoid space $\mathscr{X}=\mathscr{M}(\mathcal{A})$ is defined to be the smallest closed subset $\Gamma(\mathscr{X}) \subset \mathscr{X}$ such that every function $|f|$ for $f \in \mathcal{A}$ attains its maximum at a point of $\Gamma(\mathscr{X})$. Let $\mathfrak{X}$ be a formal analytic variety over $\operatorname{Spf}(R)$ with Raynaud generic fiber $\mathscr{X}$. If $\eta$ is a generic point of $\overline{\mathfrak{X}}$, then there is a unique preimage of $\eta$ under red which we call the Shilov point $x_{\eta}$ associated with $\eta$. The residue field of $\eta$ is isomorphic to $\widetilde{\mathscr{H}}\left(x_{\eta}\right)$. If $\mathscr{X}=\mathscr{M}(\mathcal{A})$ is affinoid, then $\Gamma(\mathscr{X})$ is the set of Shilov points of $\mathfrak{X}=\operatorname{Spf}(\mathcal{A})$.

\subsection{Analytic curves}

Following [Thu05, $\S 2.1 .3$ ], we define a (strictly) analytic curve over $K$ to be a (good) $K$-analytic space which is paracompact, of pure dimension 1 , and without boundary. The analytification of an algebraic curve over $K$ (by which we mean a 1-dimensional separated integral scheme of finite type over $K$ ) is always an analytic curve in this sense.

If $\mathscr{X}$ is an analytic curve and $\mathscr{V} \subset \mathscr{X}$ is an affinoid domain, then by [Ber90, Proposition 3.1.3] and [Thu05, Proposition 2.1.12] the following three (finite) sets coincide: (i) the topological boundary $\partial_{\text {top }} \mathscr{V}$ of $\mathscr{V}$ in $\mathscr{X}$, (ii) the boundary $\partial \mathscr{V}$ of $\mathscr{V}$ in the sense of [Ber90, $\S 2.5 .7$, and (iii) the Shilov boundary $\Gamma(\mathscr{V})$ of $\mathscr{V}$.

\subsection{Types of points in an analytic curve}

Let $x$ be a point in a $K$-analytic curve $\mathscr{X}$, and let $\mathscr{H}(x)$ be its completed residue field. The extension $\widetilde{\mathscr{H}}(x) / k$ has transcendence degree $s(x) \leqslant 1$, and the abelian group $\left|\mathscr{H}(x)^{\times}\right| /\left|K^{\times}\right|$ has rank $t(x) \leqslant 1$. Moreover, the integers $s(x)$ and $t(x)$ must satisfy the Abhyankar inequality [Vaq00, Theorem 9.2]

$$
s(x)+t(x) \leqslant 1 .
$$

Using the terminology from [Ber90] and [Ber93] (see also [Thu05, $\S 2.1$ ), we say that $x$ is type 2 if $s(x)=1$ and type 3 if $t(x)=1$. If $s(x)=t(x)=0$, then $x$ is called type 1 if $\mathscr{H}(x)=K$ and type 4 otherwise. Points of type 4 will not play any significant role in this paper. We define

$$
\begin{aligned}
\mathbf{H}_{\circ}(\mathscr{X}) & =\{\text { all points of } \mathscr{X} \text { of types } 2 \text { and } 3\}, \\
\mathbf{H}(\mathscr{X}) & =\{\text { all points of } \mathscr{X} \text { of types } 2,3, \text { and } 4\} .
\end{aligned}
$$

We call $\mathbf{H}_{\circ}(\mathscr{X})$ the set of skeletal points, because it is the union of all skeleta of admissible formal models of $\mathfrak{X}$ (see [BPR13, Corollary 5.1]), and $\mathbf{H}(\mathscr{X})$ the set of norm points of $\mathscr{X}$, because it is the set of all points corresponding to norms on the function field $K(X)$ that extend the given norm on $K$. If $\mathscr{X}=X^{\text {an }}$ is the analytification of an algebraic curve $X$ over $K$, then $X(K) \subset X^{\text {an }}$ is naturally identified with the set of type 1 points of $X^{\text {an }}$, so $\mathbf{H}\left(X^{\text {an }}\right)=X^{\text {an }} \backslash X(K)$. (Recall that we are assuming throughout this discussion that $K$ is algebraically closed.)

\subsection{Some facts about admissible $\boldsymbol{R}$-algebras}

The following fact is standard and is easily proved using the results of [BL93, $\S 1$. 


\section{BAKer, S. PAyne And J. RABinofF}

Proposition 3.7. (i) If $A$ is a finitely presented and flat $R$-algebra, then its $\varpi$-adic completion $\widehat{A}$ is an admissible $R$-algebra.

(ii) If $f: A \rightarrow B$ is a surjective homomorphism of finitely presented and flat $R$-algebras with kernel $\mathfrak{a}$, then $\widehat{f}: \widehat{A} \rightarrow \widehat{B}$ is a surjection of admissible $R$-algebras with kernel $\mathfrak{a} \widehat{A}$.

We set the following notation, which we will use until Section 3.15: $A$ and $B$ will denote admissible $R$-algebras, $\bar{A}=A \otimes_{R} k$ and $\bar{B}=B \otimes_{R} k$ their reductions, and $A_{K}=A \otimes_{R} K$ and $B_{K}=B \otimes_{R} K$ the associated $K$-affinoid algebras. We let $\mathfrak{X}=\operatorname{Spf}(A), \mathfrak{Y}=\operatorname{Spf}(B), \overline{\mathfrak{X}}=\operatorname{Spec}(\bar{A})$, and $\overline{\mathfrak{Y}}=\operatorname{Spec}(\bar{B})$. Let $f: A \rightarrow B$ be a homomorphism, let $\bar{f}: \bar{A} \rightarrow \bar{B}$ and $f_{K}: A_{K} \rightarrow B_{K}$ be the induced homomorphisms, and let $\phi: \mathfrak{Y} \rightarrow \mathfrak{X}$ and $\bar{\phi}: \overline{\mathfrak{Y}} \rightarrow \overline{\mathfrak{X}}$ be the induced morphisms.

Proposition 3.8. (i) The homomorphism $f$ is flat if and only if $\bar{f}: \bar{A} \rightarrow \bar{B}$ is flat.

(ii) The homomorphism $f$ is finite if and only if $f_{K}: A_{K} \rightarrow B_{K}$ is finite.

Proof. The 'only if' directions are clear. Suppose that $\bar{f}$ is flat. By [BL93, Lemma 1.6], it suffices to show that $f_{n}: A_{n} \rightarrow B_{n}$ is flat for all $n \geqslant 0$, where $A_{n}=A / \varpi^{n+1} A$ and $B_{n}=B / \varpi^{n+1} B$. But $A_{n}$ and $B_{n}$ are of finite presentation and flat over $R_{n}=R / \varpi^{n+1} R$, so $f_{n}$ is flat by the fibral flatness criterion [GD66, Corollaire 11.3.1].

Now suppose that $f_{K}$ is finite. Choose a surjection $\stackrel{\circ}{T}_{n} \rightarrow A$. The induced homomorphism $T_{n} \rightarrow A \otimes_{R} K \rightarrow B \otimes_{R} K$ is finite, so by [BGR84, Theorem 6.3.5/1] the composition $\stackrel{\circ}{n}_{n} \rightarrow$ $A \rightarrow\left(B \otimes_{R} K\right)^{\circ}$ is integral. Hence $A \rightarrow\left(B \otimes_{R} K\right)^{\circ}$ is integral, so $A \rightarrow B$ is integral since $B \subset\left(B \otimes_{R} K\right)^{\circ}$. Then $f_{n}: A / \varpi^{n+1} A \rightarrow B / \varpi^{n+1} B$ is of finite type and integral for all $n \geqslant 0$, so $f_{n}$ is finite, and therefore $f$ is finite by [BL93, Lemma 1.5].

Corollary 3.9. Suppose that $f_{K}: A_{K} \rightarrow B_{K}$ is finite and dominant, that is, that $\operatorname{ker}\left(f_{K}\right)$ is nilpotent. Then $\bar{f}: \bar{A} \rightarrow \bar{B}$ is finite and $\bar{\phi}: \overline{\mathfrak{Y}} \rightarrow \overline{\mathfrak{X}}$ is surjective.

Proof. Since $A \subset A_{K}$ and $B \subset B_{K}$, we have that $\operatorname{ker}(f)$ is nilpotent, and $f$ is finite by Proposition 3.8. Hence $\operatorname{Spec}(B) \rightarrow \operatorname{Spec}(A)$ is surjective, so $\overline{\mathfrak{Y}} \rightarrow \overline{\mathfrak{X}}$ is surjective. Finiteness of $f$ implies finiteness of $\bar{f}$.

We say that a ring is equidimensional of dimension $d$ provided that every maximal ideal has height $d$. Let $\mathcal{A}$ be a $K$-affinoid algebra, and let $\mathscr{X}=\mathscr{M}(\mathcal{A})$. Then $\mathcal{A}$ is equidimensional of dimension $d$ if and only if $\operatorname{dim}\left(\mathscr{O}_{\mathscr{X}, x}\right)=d$ for every $x \in \operatorname{Max} \operatorname{Spec}(\mathcal{A})$ by [BGR84, Proposition 7.3.2/8]. In particular, if $\mathscr{M}(\mathcal{B})$ is an affinoid domain in $\mathscr{M}(\mathcal{A})$ and $\mathcal{A}$ is equidimensional of dimension $d$, then so is $\mathcal{B}$.

Proposition 3.10. If $A_{K}$ is equidimensional of dimension $d$, then $\bar{A}$ is equidimensional of dimension $d$.

Proof. Replacing $\mathfrak{X}$ with an irreducible formal affine open subset, we may assume that $\mathfrak{X}$ is irreducible. Let $R\left\langle x_{1}, \ldots, x_{n}\right\rangle \rightarrow A$ be a presentation of $A$. By Noether normalization [BGR84, Theorem 6.1.2/1] we can choose the $x_{i}$ such that $K\left\langle x_{1}, \ldots, x_{d}\right\rangle \rightarrow A_{K}$ is finite and injective, where $d=\operatorname{dim}\left(A_{K}\right)$. Then $\overline{\mathfrak{X}} \rightarrow \mathbb{A}_{k}^{d}$ is finite and surjective by Corollary 3.9.

Corollary 3.11. Suppose that $f_{K}: A_{K} \rightarrow B_{K}$ is finite and dominant, and that $A_{K}$ and $B_{K}$ are equidimensional (necessarily of the same dimension). Then $\bar{\phi}: \overline{\mathbf{Y}} \rightarrow \overline{\mathfrak{X}}$ is finite and surjective, and the image of an irreducible component of $\overline{\mathfrak{Y}}$ is an irreducible component of $\overline{\mathfrak{X}}$.

Proof. This follows immediately from Proposition 3.10 and Corollary 3.9.

The following theorem uses the fact that $K$ is algebraically closed in an essential way. It can be found in [BL85, Proposition 1.1]. 


\section{NONARCHIMEDEAN GEOMETRY AND TROPICALIZATION}

Theorem 3.12. Let $\mathcal{A}$ be a $K$-affinoid algebra. Then $\mathcal{A}$ is admissible if and only if $\mathcal{A}$ is reduced.

Proof. Since $\mathcal{A}$ is always $R$-flat, by [BL93, Proposition 1.1(c)] the issue is whether $\mathcal{A}$ is topologically finitely generated. Suppose that $\mathcal{A}$ is reduced. By [BGR84, Theorem 6.4.3/1] there is a surjection $T_{n} \rightarrow \mathcal{A}$ such that the residue norm on $\mathcal{A}$ agrees with the supremum norm; then by Proposition $6.4 .3 / 3(\mathrm{i})$ of [BGR84] the induced homomorphism $\stackrel{\circ}{T}_{n} \rightarrow \mathcal{A}$ is surjective. The converse follows in a similar way from Theorem 6.4.3/1 and Corollary 6.4.3/6 of [BGR84].

Proposition 3.13. The ring $\bar{A}$ is reduced if and only if $A=\AA_{K}$, in which case $A$ is reduced.

Proof. If $A=\AA_{K}$, then $A$ is reduced by Theorem 3.12, so $\bar{A}=\widetilde{A}_{K}$ is reduced. Conversely, suppose that $\bar{A}$ is reduced. Let $\alpha: \stackrel{\circ}{T}_{n} \rightarrow A$ be a surjection. Since the $\stackrel{\circ}{T}_{n}$-ideal $\check{T}_{n}+\operatorname{ker}(\alpha)=\mathfrak{m} \check{T}_{n}+\operatorname{ker}(\alpha)$ is the kernel of the composite homomorphism $\stackrel{\circ}{n}_{n} \rightarrow A \rightarrow \bar{A}$, it is a reduced ideal; hence by [BGR84, Propositions 6.4.3/4, 6.4.3/3(i)] we have $A=\alpha\left(\stackrel{\circ}{T}_{n}\right)=\stackrel{\circ}{A}_{K}$.

Corollary 3.14. If $\bar{A}$ is an integral domain, then $A_{K}$ is an integral domain and $|\cdot|_{\sup }$ is multiplicative.

Proof. By Proposition 3.13 we have $\widetilde{A}_{K}=\bar{A}$, so the result follows from [BGR84, Proposition $6.2 .3 / 5]$.

\subsection{Finite morphisms of pure degree}

In general there is not a good notion of the 'degree' of a finite morphism $Y \rightarrow X$ between noetherian schemes when $X$ is not irreducible, since the degree of the induced map on an irreducible component of $X$ can vary from component to component. The notion of a morphism having 'pure degree' essentially means that the degree is the same on every irreducible component of $X$. This notion is quite well behaved in that it respects analytification of algebraic varieties and of admissible formal schemes. The definition of a morphism of pure degree is best formulated in the language of fundamental cycles. We refer to [Tho90] for a review of the theory of cycles on a noetherian scheme which is not necessarily of finite type over a field.

3.16 Let $X$ be a noetherian scheme. A cycle on $X$ is a finite formal sum $\sum_{W} n_{W} \cdot W$, where $n_{W} \in \mathbb{Z}$ and $W$ ranges over the irreducible closed subsets of $X$. The group of cycles on $X$ is denoted $C(X)$. The fundamental cycle of $X$ is the cycle

$$
[X]=\sum_{\zeta} \operatorname{length}_{\mathscr{O}_{X, \zeta}}\left(\mathscr{O}_{X, \zeta}\right) \cdot \overline{\{\zeta\}}
$$

where the sum is taken over all generic points of $X$. We define pushforwards and pullbacks as in intersection theory. These satisfy the usual properties; see [Tho90, Lemmas 2.4, 2.5, and 4.8].

Definition 3.17. Let $f: Y \rightarrow X$ be a finite morphism of noetherian schemes. We say that $f$ has pure degree $\delta$ and we write $[Y: X]=\delta$ provided that $f_{*}[Y]=\delta[X]$; here $\delta \in \mathbb{Q}$ need not be an integer.

Remark 3.18. Let $f: Y \rightarrow X$ be a finite morphism of noetherian schemes.

(i) If $X$ is irreducible and every generic point of $Y$ maps to the generic point of $X$, then $f$ automatically has a pure degree, which we simply call the degree of $f$. Moreover, if $X$ is integral with generic point $\zeta$, then the degree of $f$ is the dimension of $\Gamma\left(f^{-1}(\zeta), \mathscr{O}_{f^{-1}(\zeta)}\right)$ as a vector space over the function field $\mathscr{O}_{X, \zeta}$. In particular, if $f$ is a finite and dominant morphism of integral schemes, then the (pure) degree of $f$ is the degree of the extension of function fields. 


\section{BAKer, S. PAyne And J. RabinofF}

(ii) Let $\zeta$ be a generic point of $X$, and let $C=\overline{\{\zeta\}}$ be the corresponding irreducible component. Define the multiplicity of $C$ in $X$ to be the quantity

$$
\operatorname{mult}_{X}(C)=\operatorname{length}_{\mathscr{O}_{X, \zeta}}\left(\mathscr{O}_{X, \zeta}\right),
$$

so $[X]=\sum_{C} \operatorname{mult}_{X}(C) \cdot C$. It follows that $f$ has pure degree $\delta$ if and only if (i) every irreducible component $D$ of $Y$ maps to an irreducible component of $X$, and (ii) for every irreducible component $C$ of $X$ we have

$$
\delta \operatorname{mult}_{X}(C)=\sum_{D \rightarrow C} \operatorname{mult}_{Y}(D)[D: C],
$$

where $[D: C]$ is the usual degree of a finite morphism of integral schemes.

(iii) Let $g: X \rightarrow Z$ be another finite morphism of noetherian schemes. Suppose that $f$ has pure degree $\delta$ and $g$ has pure degree $\epsilon$. Then $g \circ f$ has pure degree $\delta \epsilon$.

Proposition 3.19. Let $X, Y, X^{\prime}$ be noetherian schemes, let $f: Y \rightarrow X$ be a finite morphism, let $g: X^{\prime} \rightarrow X$ be a flat morphism, let $Y^{\prime}=Y \times_{X} X^{\prime}$, and let $f^{\prime}: Y^{\prime} \rightarrow X^{\prime}$ be the projection.

(i) If $f$ has pure degree $\delta$, then $f^{\prime}$ has pure degree $\delta$.

(ii) If $g$ is surjective, then $f$ has pure degree $\delta$ if and only if $f^{\prime}$ has pure degree $\delta$.

Proof. Let $h: Y^{\prime} \rightarrow Y$ be the other projection, so $h$ is flat. We have

$$
f_{*}^{\prime}\left[Y^{\prime}\right]=f_{*}^{\prime} h^{*}[Y]=g^{*} f_{*}[Y]=\delta g^{*}[X]=\delta\left[X^{\prime}\right],
$$

which proves part (i). Conversely, suppose that $g$ is surjective (and flat) and that $f_{*}^{\prime}\left[Y^{\prime}\right]=\delta\left[X^{\prime}\right]$. Then

$$
g^{*} f_{*}[Y]=f_{*}^{\prime} h^{*}[Y]=f_{*}^{\prime}\left[Y^{\prime}\right]=\delta\left[X^{\prime}\right]=g^{*}(\delta[X]),
$$

so we are done because $g^{*}$ is visibly injective in this situation.

3.20 Next we will define pure-degree morphisms of analytic spaces. As above, we must first review the notion of the fundamental cycle of an analytic space, as defined by Gubler [Gub98, $\S 2]$.

Let $\mathscr{X}$ be a $K$-analytic space (assumed from now on to be Hausdorff and paracompact). A Zariski-closed subspace of $\mathscr{X}$ is by definition an isomorphism class of closed immersions $\mathscr{V} \hookrightarrow \mathscr{X}$. A Zariski-closed subspace of $\mathscr{X}$ is irreducible if it cannot be expressed as a union of two proper Zariski-closed subspaces. Gubler [Gub98, §2] defines a cycle on $\mathscr{X}$ to be a locally finite formal sum $\sum_{\mathscr{V}} n_{\mathscr{V}} \mathscr{V}$, where $n_{\mathscr{V}} \in \mathbb{Z}$ and $\mathscr{V}$ ranges over the irreducible Zariski-closed subspaces of $\mathscr{X}$; 'locally finite' means that there exists an admissible covering of $\mathscr{X}$ by affinoid domains intersecting only finitely many $\mathscr{V}$ with $n_{\mathscr{V}} \neq 0$. Let $C(\mathscr{X})$ denote the group of cycles on $\mathscr{X}$.

3.20.1 If $\mathscr{X}=\mathscr{M}(\mathcal{A})$ is affinoid, then the Zariski-closed subspaces of $\mathscr{X}$ are in natural inclusion-reversing bijection with the ideals of $\mathcal{A}$; therefore we have an identification $C(\mathscr{X})=$ $C(\operatorname{Spec}(\mathcal{A}))$, which we will make implicitly from now on.

3.20.2 There are natural proper pushforward and flat pullback homomorphisms for cycles on analytic spaces, which satisfy the expected properties. There is a canonical fundamental cycle $[\mathscr{X}] \in C(\mathscr{X})$ which is uniquely determined by the property that for every affinoid domain $\iota: \mathscr{M}(\mathcal{A}) \hookrightarrow \mathscr{X}$, we have $\iota^{*}[\mathscr{X}]=[\mathscr{M}(\mathcal{A})]=[\operatorname{Spec}(\mathcal{A})]$. See $[\mathrm{Gub} 98, \S \S 2.6,2.7,2.8$, and Proposition 2.12]. 


\section{NONARCHIMEDEAN GEOMETRY AND TROPICALIZATION}

Definition 3.21. Let $f: \mathscr{Y} \rightarrow \mathscr{X}$ be a finite morphism of $K$-analytic spaces. We say that $f$ has pure degree $\delta$ and we write $[\mathscr{Y}: \mathscr{X}]=\delta$ provided that $f_{*}[\mathscr{Y}]=\delta[\mathscr{X}]$. Again $\delta \in \mathbb{Q}$ need not be an integer.

Remark 3.22. Let $f: \mathscr{Y} \rightarrow \mathscr{X}$ be a finite morphism of $K$-analytic spaces.

(i) If $\mathscr{X}=\mathscr{M}(\mathcal{A})$ and $\mathscr{Y}=\mathscr{M}(\mathcal{B})$ are affinoid, then $f: \mathscr{M}(\mathcal{B}) \rightarrow \mathscr{M}(\mathcal{A})$ has pure degree $\delta$ if and only if the map of affine $\operatorname{schemes} \operatorname{Spec}(\mathcal{B}) \rightarrow \operatorname{Spec}(\mathcal{A})$ has pure degree $\delta$.

(ii) If $f$ has pure degree $\delta$ and $g: \mathscr{X} \rightarrow \mathscr{Z}$ is a finite morphism of analytic spaces of pure degree $\epsilon$, then $g \circ f$ has pure degree $\delta \epsilon$.

Proposition 3.23. Let $f: \mathscr{Y} \rightarrow \mathscr{X}$ be a finite morphism of $K$-analytic spaces.

(i) If $f$ has pure degree $\delta, \mathscr{M}(\mathcal{A}) \subset \mathscr{X}$ is an affinoid domain, and $\mathscr{M}(\mathcal{B})=f^{-1}(\mathscr{M}(\mathcal{A})$, then $\mathscr{M}(\mathcal{B}) \rightarrow \mathscr{M}(\mathcal{A})$ has pure degree $\delta$.

(ii) If there exists an admissible cover $\mathscr{X}=\bigcup_{i} \mathscr{M}\left(\mathcal{A}_{i}\right)$ of $\mathscr{X}$ by affinoid domains such that $\mathscr{M}\left(\mathcal{B}_{i}\right)=f^{-1}\left(\mathscr{M}\left(\mathcal{A}_{i}\right)\right) \rightarrow \mathscr{M}\left(\mathcal{A}_{i}\right)$ has pure degree $\delta$ for each $i$, then $f$ has pure degree $\delta$.

Proof. Since the inclusion $\mathscr{M}(\mathcal{A}) \hookrightarrow \mathscr{X}$ is flat, the first part follows as in the proof of Proposition 3.19(i). In situation (ii), let $f_{i}=\left.f\right|_{\mathscr{M}\left(\mathcal{B}_{i}\right)}: \mathscr{M}\left(\mathcal{B}_{i}\right) \rightarrow \mathscr{M}\left(\mathcal{A}_{i}\right)$, and assume $\left(f_{i}\right)_{*}\left[\mathscr{M}\left(\mathcal{B}_{i}\right)\right]=$ $\delta\left[\mathscr{M}\left(\mathcal{A}_{i}\right)\right]$ for all $i$. Arguing as in the proof of Proposition 3.19(i), we see that the pullback of $f_{*}[\mathscr{Y}]$ to $\mathscr{M}\left(\mathcal{A}_{i}\right)$ is equal to $\delta\left[\mathscr{M}\left(\mathcal{A}_{i}\right)\right]$ for all $i$; since $[\mathscr{X}]$ is the unique cycle which pulls back to $\left[\mathscr{M}\left(\mathcal{A}_{i}\right)\right]$ for all $i$, this shows $f_{*}[\mathscr{Y}]=\delta[\mathscr{X}]$.

The property of being a finite morphism of pure degree is compatible with analytification.

Proposition 3.24. Let $f: Y \rightarrow X$ be a morphism of finite-type $K$-schemes. Then $f$ is finite of pure degree $\delta$ if and only if $f^{\text {an }}: Y^{\text {an }} \rightarrow X^{\text {an }}$ is finite of pure degree $\delta$.

Proof. By [Con99, Theorem A.2.1], $f$ is finite if and only if $f^{\text {an }}$ is finite. Hence we may assume that $X=\operatorname{Spec}(A)$ and $Y=\operatorname{Spec}(B)$ are affine. If $\mathscr{M}(\mathcal{A}) \subset X^{\text {an }}$ is an affinoid domain, then $\operatorname{Spec}(\mathcal{A}) \rightarrow \operatorname{Spec}(A)$ is flat by Lemma A.1.2 of [Con99], and if $\left\{\mathscr{M}\left(\mathcal{A}_{i}\right)\right\}_{i \in I}$ is an admissible covering of $X^{\text {an }}$, then $\coprod_{i \in I} \operatorname{Spec}\left(\mathcal{A}_{i}\right) \rightarrow \operatorname{Spec}(A)$ is flat and surjective. Let $\mathscr{M}\left(\mathcal{B}_{i}\right)=f^{-1}\left(\mathscr{M}\left(\mathcal{A}_{i}\right)\right)$. We claim that $\mathcal{B}_{i}=B \otimes_{A} \mathcal{A}_{i}$. Since $B \otimes_{A} \mathcal{A}_{i}$ is finite over $\mathcal{A}_{i}$, it is affinoid by [BGR84, Proposition $6.1 .1 / 6]$, so the claim follows easily from the universal property of the analytification (see also [Con99, § A.2]). Hence by Proposition 3.19(ii), $f$ has pure degree $\delta$ if and only if $\operatorname{Spec}\left(\mathcal{B}_{i}\right) \rightarrow \operatorname{Spec}\left(\mathcal{A}_{i}\right)$ has pure degree $\delta$ for each $i$; by Remark 3.22(i), this is the case if and only if $\mathscr{M}\left(\mathcal{B}_{i}\right) \rightarrow \mathscr{M}\left(\mathcal{A}_{i}\right)$ has pure degree $\delta$ for each $i$, which is equivalent to $f^{\text {an }}$ having pure degree $\delta$ by Proposition 3.23(ii).

The following counterpart to Proposition 3.24 allows us to compare the degrees of the generic and special fibers of a finite morphism of admissible formal schemes. It will play a key role throughout this paper.

Proposition 3.25 (Projection formula). Let $f: \mathfrak{Y} \rightarrow \mathfrak{X}$ be a finite morphism of admissible formal schemes, and let $f^{\text {an }}: \mathfrak{Y}^{\text {an }} \rightarrow \mathfrak{X}^{\text {an }}$ and $\bar{f}: \overline{\mathfrak{Y}} \rightarrow \overline{\mathfrak{X}}$ be the induced morphisms on the generic and special fibers, respectively. If $f^{\text {an }}$ has pure degree $\delta$, then $\bar{f}$ has pure degree $\delta$.

Proof. The theory of cycles on analytic spaces discussed above is part of Gubler's more general intersection theory on admissible formal schemes, and our 'projection formula' is in fact a special case of Gubler's projection formula [Gub98, Proposition 4.5]; this can be seen as follows. Choose any $\varpi \in K^{\times}$with $\operatorname{val}(\varpi) \in(0, \infty)$, and let $D$ be the Cartier divisor on $\mathfrak{X}$ defined by $\varpi$. 


\section{BAKer, S. PAyne And J. RabinofF}

Essentially by definition (cf. (3.8) and (3.10) of [Gub98]) the intersection product $D$. [ $\mathfrak{X}^{\text {an }}$ ] is equal to $\operatorname{val}(\varpi)[\overline{\mathfrak{X}}]$, and likewise $\left(f^{*} D\right) \cdot\left[\mathfrak{Y}^{\text {an }}\right]=\operatorname{val}(\varpi)[\overline{\mathfrak{Y}}]$. Hence if $f_{*}^{\text {an }}\left[\mathfrak{Y}^{\text {an }}\right]=\delta\left[\mathfrak{X}^{\text {an }}\right]$, then

$$
\operatorname{val}(\varpi) \bar{f}_{*}[\overline{\mathfrak{Y}}]=f_{*}\left(\left(f^{*} D\right) \cdot\left[\mathfrak{Y}^{\mathrm{an}}\right]\right)=D \cdot f_{*}^{\text {an }}\left[\mathfrak{Y}^{\mathrm{an}}\right]=D \cdot\left(\delta\left[\mathfrak{X}^{\mathrm{an}}\right]\right)=\operatorname{val}(\varpi) \delta[\overline{\mathfrak{X}}],
$$

where the second equality is by Gubler's projection formula. Canceling the factors of $\operatorname{val}(\varpi)$ yields Proposition 3.25.

Remark 3.26. The converse to Proposition 3.25 does not hold in general. The following example is due to Gubler: let $\mathfrak{X}=\operatorname{Spf}(R[x] /(x(x-\varpi)))$ and $\mathfrak{Y}=\mathfrak{X} \amalg \operatorname{Spf}(R)$, and let $f: \mathfrak{Y} \rightarrow \mathfrak{X}$ be the map which is the identity on $\mathfrak{X}$ and which maps $\operatorname{Spf}(R)$ to $\mathfrak{X}$ via $x \mapsto 0$. Then $f$ an does not have a pure degree, but $\bar{f}$ does since $\overline{\mathfrak{X}}$ is a point.

3.27 Here we note some special cases of the projection formula:

(i) Suppose that $\mathfrak{X}=\operatorname{Spf}(A)$ and $\mathfrak{Y}=\operatorname{Spf}(B)$, and that $A$ is an integral domain with fraction field $Q$. If all generic points of $\operatorname{Spec}\left(B \otimes_{R} K\right)$ map to the generic point of $\operatorname{Spec}\left(A \otimes_{R} K\right)$, then $\mathscr{M}\left(B \otimes_{R} K\right) \rightarrow \mathscr{M}\left(A \otimes_{R} K\right)$ is finite with pure degree equal to $\operatorname{dim}_{Q}\left(B \otimes_{A} Q\right)$. By (3.18.1), for every irreducible component $\overline{\mathfrak{C}}$ of $\overline{\mathfrak{X}}$ we have

$$
\operatorname{dim}_{Q}\left(B \otimes_{A} Q\right) \cdot \operatorname{mult}_{\overline{\mathfrak{X}}}(\overline{\mathfrak{C}})=\sum_{\overline{\mathfrak{D}} \rightarrow \overline{\mathfrak{C}}} \operatorname{mult}_{\overline{\mathfrak{Y}}}(\overline{\mathfrak{D}}) \cdot[\overline{\mathfrak{D}}: \overline{\mathfrak{C}}]
$$

where the sum is taken over all irreducible components $\overline{\mathfrak{D}}$ of $\overline{\mathfrak{Y}}$ that surject onto $\overline{\mathfrak{C}}$.

(ii) Suppose that $f^{\text {an }}: \mathfrak{Y}^{\text {an }} \rightarrow \mathfrak{X}^{\text {an }}$ is an isomorphism. Then for every irreducible component $\mathfrak{C}$ of $\overline{\mathfrak{X}}$ we have

$$
\text { mult }_{\overline{\mathfrak{X}}}(\overline{\mathfrak{C}})=\sum_{\overline{\mathfrak{D}} \rightarrow \overline{\mathfrak{C}}} \operatorname{mult}_{\overline{\mathfrak{Y}}}(\overline{\mathfrak{D}}) \cdot[\overline{\mathfrak{D}}: \overline{\mathfrak{C}}]
$$

where the sum is taken over all irreducible components $\overline{\mathfrak{D}}$ of $\overline{\mathfrak{Y}}$ that surject onto $\overline{\mathfrak{C}}$, because an isomorphism has pure degree 1.

\section{Tropical integral models}

We continue to assume that $K$ is an algebraically closed field which is complete with respect to a nontrivial nonarchimedean valuation.

Notation 4.1. Let $M \cong \mathbb{Z}^{n}$ be a lattice, with dual lattice $N=\operatorname{Hom}(M, \mathbb{Z})$. If $H$ is an additive subgroup of $\mathbb{R}$, we write $M_{H}$ for $M \otimes_{\mathbb{Z}} H$, so $N_{H}$ is naturally identified with $\operatorname{Hom}(M, H)$. We write $\langle\cdot, \cdot\rangle$ to denote the canonical pairings $M \times N \rightarrow \mathbb{Z}$ and $M_{\mathbb{R}} \times N_{\mathbb{R}} \rightarrow \mathbb{R}$.

Let $\mathbf{T}=\operatorname{Spec} K[M]$ be the torus over $K$ with character lattice $M$. For $u$ in $M$, we write $x^{u}$ for the corresponding character, considered as a function in $K[M]$.

\subsection{Extended tropicalization}

A point $\|\cdot\|$ in $\mathbf{T}^{\text {an }}$ naturally determines a real-valued linear function on the character lattice $M$, taking $u$ to $-\log \left\|x^{u}\right\|$. The induced tropicalization map trop: $\mathbf{T}^{\text {an }} \rightarrow N_{\mathbb{R}}$ is continuous, proper, and surjective [Pay09a]. The image of $\mathbf{T}(K)$ is exactly $N_{G}$, which is dense in $N_{\mathbb{R}}$ because $G$ is nontrivial and divisible.

More generally, if $\sigma$ is a pointed rational polyhedral cone in $N_{\mathbb{R}}$ and $Y_{\sigma}=\operatorname{Spec} K\left[\sigma^{\vee} \cap M\right]$ is the associated affine toric variety with dense torus $\mathbf{T}$, then there is a natural tropicalization 


\section{NONARCHIMEDEAN GEOMETRY AND TROPICALIZATION}

map from $Y_{\sigma}$ to the space of additive semigroup homomorphisms $\operatorname{Hom}\left(\sigma^{\vee} \cap M, \mathbb{R} \cup\{\infty\}\right)$ taking a point $\|\cdot\|$ to the semigroup map $u \mapsto-\log \left\|x^{u}\right\|$, where $-\log (0)$ is defined to be $\infty$. See [Pay09a, Rab12] for further details. We write $N_{\mathbb{R}}(\sigma)$ for the image of $Y_{\sigma}^{\text {an }}$ under this extended tropicalization map.

Definition 4.3. We say that a point in $N_{\mathbb{R}}(\sigma)$ is $G$-rational if it is in the subspace $\operatorname{Hom}\left(\sigma^{\vee} \cap M\right.$, $G \cup\{\infty\})$. Note that the image of any $K$-rational point of $Y_{\sigma}$ is $G$-rational.

For any toric variety $Y_{\Delta}$, the tropicalization $N_{\mathbb{R}}(\Delta)$ is the union of the spaces $N_{\mathbb{R}}(\sigma)$ for $\sigma$ in $\Delta$, glued along the open inclusions $N_{\mathbb{R}}(\tau) \subset N_{\mathbb{R}}(\sigma)$ for $\tau \preceq \sigma$. The tropicalization maps on torusinvariant affine opens are compatible with this gluing, and together give a natural continuous, proper, and surjective map of topological spaces trop: $Y_{\Delta}^{\text {an }} \rightarrow N_{\mathbb{R}}(\Delta)$. Note that the vector space $N_{\mathbb{R}}$, which is the tropicalization of the dense torus $\mathbf{T} \subset Y_{\Delta}$, is open and dense in $N_{\mathbb{R}}(\Delta)$. For the purpose of constructing tropical integral models of toric varieties and their subvarieties, it will generally suffice to study polyhedral complexes in $N_{\mathbb{R}}$.

Let $X$ be a closed subscheme of $Y_{\Delta}$. The tropicalization $\operatorname{Trop}(X)$ is the image of $X^{\text {an }}$ under trop. Since $X(K)$ is dense in $X^{\text {an }}$, its image is dense in $\operatorname{Trop}(X)$. Furthermore, every $G$-rational point of $\operatorname{Trop}(X)$ is the image of a point of $X(K)$, and if $X$ is irreducible, then the preimage of any point in $\operatorname{Trop}(X) \cap N_{G}$ is Zariski dense in $X$. See [Pay09b, Corollary 4.2] and [Pay12, Remark 2], [Gub13, Proposition 4.14], or [OP13, Theorem 4.2.5].

\subsection{Polyhedral domains}

Recall that the recession cone $\sigma_{P}$ of a nonempty polyhedron $P \subset N_{\mathbb{R}}$ is the set of those $v$ in $N_{\mathbb{R}}$ such that $w+v$ is in $P$ whenever $w$ is in $P$. If $P$ is the intersection of the halfspaces $\left\langle u_{1}, v\right\rangle \geqslant a_{1}$, $\ldots,\left\langle u_{r}, v\right\rangle \geqslant a_{r}$, then $\sigma_{P}$ is the dual of the cone in $M_{\mathbb{R}}$ spanned by $u_{1}, \ldots, u_{r}$. In particular, if $P$ is an integral $G$-affine polyhedron, then these halfspaces can be chosen with each $u_{i}$ in $M$, so the recession cone $\sigma_{P}$ is a rational polyhedral cone. The recession cone can also be characterized as the intersection with $N_{\mathbb{R}} \times\{0\}$ of the closure in $N_{\mathbb{R}} \times \mathbb{R}$ of the cone spanned by $P \times\{1\}$.

Let $P$ be an integral $G$-affine polyhedron in $N_{\mathbb{R}}$ that does not contain any positive-dimensional affine linear subspace, so its recession cone $\sigma=\sigma_{P}$ is pointed.

Definition 4.5. The polyhedral domain associated with $P$ is the inverse image of the closure of $P$ in $N_{\mathbb{R}}(\sigma)$ under trop: $Y_{\sigma}^{\text {an }} \rightarrow N_{\mathbb{R}}(\sigma)$ and is denoted $\mathscr{U}^{P}$.

These polyhedral domains, introduced in [Rab12], directly generalize the polytopal domains studied by Gubler in [Gub07b]. Indeed, a polytopal domain is the preimage in $\mathbf{T}^{\text {an }}$ of an integral $G$-affine polytope in $N_{\mathbb{R}}$. Since the recession cone of a polytope in $N_{\mathbb{R}}$ is the zero cone, whose associated toric variety is T, Gubler's polytopal domains are exactly the special case of these polyhedral domains where $P$ is bounded.

By $[$ Rab12, $\S 6]$ the polyhedral domain $\mathscr{U}^{P}$ is an affinoid domain in $Y_{\sigma}^{\text {an }}$ with coordinate ring

$$
K\left\langle\mathscr{U}^{P}\right\rangle=\left\{\sum_{u \in \sigma^{\vee} \cap M} a_{u} x^{u}: \lim \left(\operatorname{val}\left(a_{u}\right)+\langle u, v\rangle\right)=\infty \text { for all } v \in P\right\},
$$

where the limit is taken over all complements of finite sets. Its supremum norm is given by

$$
\left|\sum a_{u} x^{u}\right|_{\sup }=\sup _{\substack{u \in \sigma^{\vee} \cap M \\ v \in P}}\left|a_{u}\right| \exp (-\langle u, v\rangle) .
$$




\section{BAKer, S. PAyne And J. RabinofF}

Since the recession cone $\sigma$ is pointed, the polyhedron $P$ contains no linear subspace and hence has vertices. The supremum above is always achieved at one of the vertices of $P$, so the ring of power-bounded regular functions on $\mathscr{U}^{P}$ is

$$
K\left\langle\mathscr{U}^{P}\right\rangle^{\circ}=\left\{\sum_{u \in \sigma^{\vee} \cap M} a_{u} x^{u} \in K\left\langle\mathscr{U}^{P}\right\rangle: \operatorname{val}\left(a_{u}\right)+\langle u, v\rangle \geqslant 0 \text { for all } v \in \operatorname{vert}(P)\right\} .
$$

Since $K\left\langle\mathscr{U}^{P}\right\rangle$ is reduced, Theorem 3.12 implies that $\mathfrak{U}^{P}=\operatorname{Spf}\left(K\left\langle\mathscr{U}^{P}\right\rangle^{\circ}\right)$ is an admissible formal scheme with analytic generic fiber $\mathscr{U}^{P}$.

Remark 4.6. If $P$ is integral affine but not $G$-affine, then the inverse image $\mathscr{U}^{P}$ of the closure of $P$ under trop is a nonstrict affinoid domain. Indeed, if $K^{\prime}$ is a complete valued field extension of $K$ whose value group $G^{\prime}$ is large enough that $P$ is $G^{\prime}$-affine, then $\mathscr{U}^{P} \widehat{\otimes}_{K} K^{\prime}$ is strictly $K^{\prime}$-affinoid.

\subsection{Polyhedral integral models}

Let $P$ be an integral $G$-affine polyhedron in $N_{\mathbb{R}}$ whose recession cone $\sigma=\sigma_{P}$ is pointed. As usual, we let $Y_{\sigma}=$ Spec $K\left[\sigma^{\vee} \cap M\right]$ denote the associated affine toric variety with dense torus $\mathbf{T}$.

Definition 4.8. We define $R\left[Y^{P}\right] \subset K\left[\sigma^{\vee} \cap M\right]$ to be the subring consisting of those Laurent polynomials $\sum a_{u} x^{u}$ such that $\operatorname{val}\left(a_{u}\right)+\langle u, v\rangle \geqslant 0$ for all $v \in P$ and all $u$. The scheme $Y^{P}:=$ $\operatorname{Spec}\left(R\left[Y^{P}\right]\right)$ is called a polyhedral integral model of $Y_{\sigma}$.

In other words, $R\left[Y^{P}\right]$ is the intersection of $K\left\langle\mathscr{U}^{P}\right\rangle^{\circ}$ with $K[M]$. It is clear that $K\left\langle\mathscr{U}^{P}\right\rangle^{\circ}$ is the $\varpi$-adic completion of $R\left[Y^{P}\right]$. Note that $R\left[Y^{P}\right]$ is torsion-free and hence flat over $R$.

Lemma 4.9. The tensor product $R\left[Y^{P}\right] \otimes_{R} K$ is equal to $K\left[Y_{\sigma}\right]$.

Proof. By definition we have $R\left[Y^{P}\right] \otimes_{R} K \subset K\left[Y_{\sigma}\right]$. For the other inclusion, note that if $g=$ $\sum b_{u} x^{u}$ is in $K\left[Y_{\sigma}\right]$, then the minimum over $v$ in $P$ of $\operatorname{val}\left(b_{u}\right)+\langle u, v\rangle$ is achieved at some vertex of $P$. It follows that some sufficiently high power of $\varpi$ times $g$ is in $R\left[Y^{P}\right]$, and hence $g$ is in $R\left[Y^{P}\right] \otimes_{R} K$.

Remark 4.10. One could equivalently define $R\left[Y^{P}\right]$ to be the subring of $K[M]$ satisfying the same inequalities. Since $P$ is closed under addition of points in $\sigma$, any Laurent polynomial satisfying these inequalities for all $v$ in $P$ must be supported in $\sigma^{\vee}$.

We will use the following notation in the proof of Proposition 4.11. For each face $F \leqslant P$, let $\sigma(F)$ be the cone in $N_{\mathbb{R}}$ spanned by $P-v$ for any $v$ in the relative interior of $F$. In other words, $\sigma(F)=\operatorname{Star}_{P}(F)$. We fix a labeling $v_{1}, \ldots, v_{r}$ for the vertices of $P$, and write $\sigma_{i}$ for $\sigma\left(v_{i}\right)$. The dual cone $\sigma_{i}^{\vee}$ is

$$
\sigma_{i}^{\vee}=\left\{u \in \sigma_{P}^{\vee}:\left\langle u, v_{i}\right\rangle \leqslant\left\langle u, v_{j}\right\rangle \text { for all } j\right\} .
$$

The cones $\sigma_{1}^{\vee}, \ldots, \sigma_{r}^{\vee}$ are the maximal cones of the (possibly degenerate) inner normal fan of $P$, and their union is $\sigma_{P}^{\vee}$.

Proposition 4.11. Let $P$ be a $G$-rational polyhedron in $N_{\mathbb{R}}$. Then $R\left[Y^{P}\right]$ is finitely presented over $R$.

Proof. By [RG71, Corollary 3.4.7], any finitely-generated and flat algebra over an integral domain is automatically of finite presentation, so it suffices to show that $R\left[Y^{P}\right]$ is finitely generated.

The cones $\sigma_{1}^{\vee}, \ldots, \sigma_{r}^{\vee}$ cover $\sigma^{\vee}$, so $R\left[Y^{P}\right]$ is generated by the subrings

$$
A_{j}=R\left[Y^{P}\right] \cap K\left[\sigma_{j}^{\vee} \cap M\right]
$$




\section{NONARCHIMEDEAN GEOMETRY AND TROPICALIZATION}

for $1 \leqslant j \leqslant r$. Therefore, it will suffice to show that each $A_{j}$ is finitely generated over $R$.

The semigroup $\sigma_{j}^{\vee} \cap M$ is finitely generated by Gordan's lemma [Ful93, $\S 1.2$, Proposition 1]. Let $u_{1}, \ldots, u_{s}$ be generators, and choose $a_{1}, \ldots, a_{s}$ in $K^{\times}$such that $\operatorname{val}\left(a_{i}\right)+\left\langle u_{i}, v_{j}\right\rangle=0$. Then each monomial in $A_{j}$ can be written as an element of $R$ times a monomial in the $a_{i} x^{u_{i}}$. It follows that $A_{j}$ is finitely generated over $R$, as required, with generating set $\left\{a_{1} x^{u_{1}}, \ldots, a_{s} x^{u_{s}}\right\}$.

In particular, $Y^{P}$ is a flat and finitely-presented $R$-model of the affine toric variety $Y_{\sigma}$.

Remark 4.12. As in Remark 4.6, one can construct an algebraic model $Y^{P}$ of $Y_{\sigma}$ associated with an integral affine but not $G$-affine polyhedron $P$; when $P$ is a point this is done in [OP13]. This model is not of finite type.

\subsection{Polyhedral integral and formal models of subschemes}

Let $P$ be an integral $G$-affine polyhedron with pointed recession cone $\sigma$. Let $X$ be the closed subscheme of the affine toric variety $Y_{\sigma}$ over $K$ defined by an ideal $\mathfrak{a} \subset K\left[Y_{\sigma}\right]$.

Definition 4.14. (i) Let $\mathscr{X}^{P}=X^{\text {an }} \cap \mathscr{U}^{P}$. This is the Zariski-closed subspace of $\mathscr{U}^{P}$ defined by $\mathfrak{a} K\left\langle\mathscr{U}^{P}\right\rangle$.

(ii) The polyhedral integral model of $X$ is the scheme-theoretic closure $X^{P}$ of $X$ in $Y^{P}$. It is defined by the ideal $\mathfrak{a}^{P}=\mathfrak{a} \cap R\left[Y^{P}\right]$.

(iii) The polyhedral formal model of $\mathscr{X}^{P}$ is the $\varpi$-adic completion $\mathfrak{X}^{P}$ of $X^{P}$. We will show in Proposition 4.17 that $\mathfrak{X}^{P}$ is an admissible formal scheme with generic fiber $\mathscr{X}^{P}$.

(iv) The canonical model of $\mathscr{X}^{P}$ is

$$
\mathfrak{X}_{\text {can }}^{P}=\operatorname{Spf}\left(\left(K\left\langle\mathscr{U}^{P}\right\rangle / \mathfrak{a} K\left\langle\mathscr{U}^{P}\right\rangle\right)^{\circ}\right) .
$$

By Theorem 3.12, the canonical model is admissible if and only if $\mathscr{X}^{P}$ is reduced.

Notation 4.15. The P-initial degeneration of $X$ is defined to be

$$
\operatorname{in}_{P}(X)=X^{P} \otimes_{R} k=\mathfrak{X}^{P} \otimes_{R} k .
$$

As usual we write $\overline{\mathfrak{X}}_{\text {can }}^{P}=\mathfrak{X}_{\text {can }}^{P} \otimes_{R} k$. This coincides with the canonical reduction of $\mathscr{X}^{P}$ when $\mathscr{X}^{P}$ is reduced. In the case where $P$ is a single point $w \in N_{G}$ we write $\mathscr{X}^{w}, \mathfrak{X}^{w}, \operatorname{in}_{w}(X)$, etc. In this case, $\operatorname{in}_{w}(X)$ is the $w$-initial degeneration of $X$ in the sense generally used in the literature (and in the introduction).

Lemma 4.16. The ideal $\mathfrak{a}^{P}$ is finitely generated.

Proof. Since $X^{P}$ is the closure of its generic fiber, it is flat over $\operatorname{Spec} R$, and its coordinate ring is a quotient of the finitely-generated $R$-algebra $R[M]$. Since any finitely-generated flat algebra over an integral domain is finitely presented [RG71, Corollary 3.4.7], it follows that $\mathfrak{a}^{P}$ is finitely generated.

Proposition 4.17. The formal scheme $\mathfrak{X}^{P}$ is the formal closed subscheme of $\mathfrak{U}^{P}$ defined by $\mathfrak{a}^{P} K\left\langle\mathscr{U}^{P}\right\rangle^{\circ}$. It is an admissible formal scheme with generic fiber $\mathscr{X}^{P}$ and special fiber $\operatorname{in}_{P}(X)$.

Proof. The admissibility of $\mathfrak{X}^{P}$ is a consequence of Proposition 3.7(i). If $A=R\left[Y^{P}\right] / \mathfrak{a}$, then by definition $X^{P}=\operatorname{Spec}(A)$ and $\mathfrak{X}^{P}=\operatorname{Spf}(\widehat{A})$, where $\widehat{A}$ is the $\varpi$-adic completion of $A$. By Proposition 3.7(ii) the sequence

$$
0 \longrightarrow \mathfrak{a}^{P} K\left\langle\mathscr{U}^{P}\right\rangle^{\circ} \longrightarrow K\left\langle\mathscr{U}^{P}\right\rangle^{\circ} \longrightarrow \widehat{A} \longrightarrow 0
$$




\section{BAKer, S. PAyne And J. RabinofF}

is exact; it follows that $\mathfrak{X}^{P}$ is the closed subscheme of $\mathfrak{U}^{P}$ defined by $\mathfrak{a}^{P} K\left\langle\mathscr{U}^{P}\right\rangle^{\circ}$. We have

$$
\left(K\left\langle\mathscr{U}^{P}\right\rangle^{\circ} / \mathfrak{a}^{P} K\left\langle\mathscr{U}^{P}\right\rangle^{\circ}\right) \otimes_{R} K=K\left\langle\mathscr{U}^{P}\right\rangle / \mathfrak{a} K\left\langle\mathscr{U}^{P}\right\rangle
$$

since $K \mathfrak{a}^{P}=\mathfrak{a}$, so $\mathfrak{X}^{P} \otimes_{R} K=\mathscr{X}^{P}$. The special fiber of $\mathfrak{X}^{P}$ agrees with the special fiber of $X^{P}$ by construction.

The canonical inclusion

$$
K\left\langle\mathscr{U}^{P}\right\rangle^{\circ} / \mathfrak{a}^{P} K\left\langle\mathscr{U}^{P}\right\rangle^{\circ} \hookrightarrow\left(K\left\langle\mathscr{U}^{P}\right\rangle / \mathfrak{a} K\left\langle\mathscr{U}^{P}\right\rangle\right)^{\circ}
$$

induces a map of formal schemes

$$
\mathfrak{X}_{\text {can }}^{P} \longrightarrow \mathfrak{X}^{P} .
$$

As this morphism induces an isomorphism on analytic generic fibers, it is finite when $\mathscr{X}^{P}$ is reduced by Proposition 3.8(ii). Its special fiber is a morphism $\overline{\mathfrak{X}}_{\text {can }}^{P} \rightarrow \operatorname{in}_{P}(X)$. Many of the results of this paper are proved by using this morphism and the results of Section 3 (in particular the projection formula, Proposition 3.25) to compare these two models.

\subsection{Compatibility with extension of the ground field}

We continue to use the notation of Section 4.13. Let $K^{\prime}$ be an algebraically closed complete valued field extension of $K$, with valuation ring $R^{\prime}$ and residue field $k^{\prime}$. Let $P$ be an integral $G$-affine polyhedron in $N_{\mathbb{R}}$ with pointed recession cone $\sigma$. Let $Y_{\sigma}^{\prime}=Y_{\sigma} \otimes_{K} K^{\prime}$, so $Y_{\sigma}^{\prime}$ is the affine toric variety defined over $K^{\prime}$ with dense torus $\mathbf{T}^{\prime}:=\mathbf{T} \otimes_{K} K^{\prime}$ associated with the cone $\sigma$. The triangle

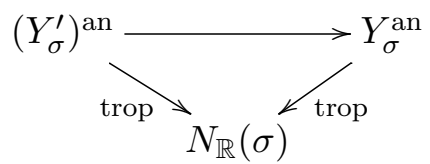

commutes, so $\mathscr{U}^{P} \widehat{\otimes}_{K} K^{\prime}$ is the polyhedral domain in $\left(Y_{\sigma}^{\prime}\right)^{\text {an }}$ associated with $P$. Likewise the polyhedral integral model $\left(Y^{\prime}\right)^{P}$ of $Y_{\sigma}^{\prime}$ associated with $P$ is naturally identified with $Y^{P} \otimes_{R} R^{\prime}$. Indeed, as an $R$-module we have

$$
R\left[Y^{P}\right]=\bigoplus_{u \in \sigma^{\vee} \cap M} R_{u} \cdot x^{u} \subset K\left[\sigma^{\vee} \cap M\right], \quad \text { where } \quad R_{u}=\left\{a \in R: \operatorname{val}(a) \geqslant \max _{v \in \operatorname{vert}(P)}-\langle u, v\rangle\right\} .
$$

Since $\langle u, v\rangle \in G$ for all $u \in M$ and $v \in \operatorname{vert}(P)$, each $R_{u}$ is a free $R$-module of rank 1 , so the image of $R_{u} \otimes_{R} R^{\prime}$ in $K^{\prime}$ is exactly $R_{u}^{\prime}$.

Let $X \subset Y_{\sigma}$ be the closed subscheme defined by an ideal $\mathfrak{a} \subset K\left[\sigma^{\vee} \cap M\right]$, and let $X^{\prime}=$ $X \otimes_{K} K^{\prime} \subset Y_{\sigma}^{\prime}$, so $X^{\prime}$ is defined by $\mathfrak{a} K^{\prime}\left[\sigma^{\vee} \cap M\right]$. Since the above triangle is commutative, we have $\operatorname{Trop}(X)=\operatorname{Trop}\left(X^{\prime}\right) \subset N_{\mathbb{R}}(\sigma)$, and trop: $\left(X^{\prime}\right)^{\text {an }} \rightarrow \operatorname{Trop}(X)$ factors through the natural $\operatorname{map}\left(X^{\prime}\right)^{\text {an }} \rightarrow X^{\text {an }}$. Hence

$$
\left(\mathscr{X}^{\prime}\right)^{P}=\operatorname{trop}^{-1}(P) \cap\left(X^{\prime}\right)^{\text {an }}=\mathscr{X}^{P} \widehat{\otimes}_{K} K^{\prime} .
$$

Since schematic closure commutes with flat base change, the polyhedral integral model $\left(X^{\prime}\right)^{P}$ of $X^{\prime}$ coincides with $X^{P} \otimes_{R} R^{\prime}$; hence if $\mathfrak{a}^{P}=\mathfrak{a} \cap R\left[Y^{P}\right]$ is the ideal defining $X^{P}$, then $\left(X^{\prime}\right)^{P}$ is defined by $\mathfrak{a}^{P} R^{\prime}\left[\left(Y^{\prime}\right)^{P}\right]$. It follows from this and Proposition 4.17 that $\left(\mathfrak{X}^{\prime}\right)^{P}=\mathfrak{X}^{P} \widehat{\otimes}_{R} R^{\prime}$, and in particular that $\operatorname{in}_{P}\left(X^{\prime}\right)=\operatorname{in}_{P}(X) \otimes_{k} k^{\prime}$. As for the canonical models, suppose that $X$ is reduced, so $X^{\prime}$ is reduced as well. Then $\left(\mathfrak{X}^{\prime}\right)_{\text {can }}^{P}=\mathfrak{X}_{\text {can }}^{P} \widehat{\otimes}_{R} R^{\prime}$ because $\left(\mathfrak{X}_{\text {can }}^{P} \widehat{\otimes}_{R} R^{\prime}\right) \otimes_{R^{\prime}} k^{\prime}=\left(\mathfrak{X}_{\text {can }}^{P} \otimes_{R} k\right) \otimes_{k} k^{\prime}$ is reduced; cf. Proposition 3.13 . 


\section{NONARCHIMEDEAN GEOMETRY AND TROPICALIZATION}

Below we will make various definitions by passing to a valued field extension $K^{\prime}$ of $K$. In order for these definitions to be independent of the choice of $K^{\prime}$, we will need the following fact, proven in [Duc09, $\S 0.3 .2]$ or [Con08, $\S 4]$.

Lemma 4.19. Let $K_{1}$ and $K_{2}$ be complete valued field extensions of $K$. Then there is a complete valued field extension $K^{\prime}$ of $K$ admitting isometric embeddings $K_{1} \hookrightarrow K^{\prime}$ and $K_{2} \hookrightarrow K^{\prime}$ over $K$.

\subsection{Relative multiplicities and tropical multiplicities}

Recall Section 3.3 that if $\mathscr{X}=\mathscr{M}(\mathcal{A})$ is an affinoid space, then the reduction map induces a one-to-one correspondence between the Shilov boundary points of $\mathscr{X}^{\text {an }}$ and the generic points of the canonical reduction $\operatorname{Spec}(\widetilde{\mathcal{A}})$. This leads to the following definition.

Definition 4.21. Let $X \subset \mathbf{T}$ be a reduced and equidimensional closed subscheme, let $x \in X^{\text {an }}$, let $w=\operatorname{trop}(x)$, and suppose $w \in N_{G}$. Define the relative multiplicity $m_{\mathrm{rel}}(x)$ of $x$ in $\operatorname{trop}^{-1}(w)$ as follows. If $x$ is not a Shilov boundary point of $\operatorname{trop}^{-1}(w)$, then we define its multiplicity to be 0 . Otherwise $\operatorname{red}(x)$ is the generic point of an irreducible component $\overline{\mathfrak{C}}$ of $\overline{\mathfrak{X}}_{\text {can }}^{w}$; we define the multiplicity of $x$ to be $[\overline{\mathfrak{C}}: \operatorname{im}(\overline{\mathfrak{C}})]$, where $\operatorname{im}(\overline{\mathfrak{C}})$ is the image of $\overline{\mathfrak{C}}$ in $\operatorname{in}_{w}(X)$ (this is an irreducible component by Corollary 3.11).

Now suppose $w \notin N_{G}$. Let $K^{\prime}$ be an algebraically closed complete valued field extension of $K$ such that $w \in N_{G^{\prime}}$, where $G^{\prime}$ is the value group of $K^{\prime}$. Let $X^{\prime}=X \otimes_{K} K^{\prime}$, and let $\phi:\left(X^{\prime}\right)^{\text {an }} \rightarrow X^{\text {an }}$ be the natural morphism. We define

$$
m_{\mathrm{rel}}(x)=\sum_{x^{\prime} \in \phi^{-1}(x)} m_{\mathrm{rel}}\left(x^{\prime}\right) .
$$

In order for this definition to make sense, by Lemma 4.19 we only have to show that if $K \subset K^{\prime} \subset K^{\prime \prime}$ are algebraically closed complete valued field extensions, then we can calculate $m_{\text {rel }}(x)$ with respect to either $K^{\prime}$ or $K^{\prime \prime}$. Replacing $K$ with $K^{\prime}$, we are reduced to showing the following.

LEMMA 4.22. Let $K^{\prime}$ be an algebraically closed complete valued field extension of $K$, and let $X^{\prime}=$ $X \otimes_{K} K^{\prime}$. Let $x \in X^{\text {an }}$, let $w=\operatorname{trop}(x)$, and suppose $w \in N_{G}$. Then the natural map $\left(\mathscr{X}^{\prime}\right)^{w} \rightarrow$ $\mathscr{X}^{w}$ induces a bijection of Shilov boundary points which preserves relative multiplicities.

Proof. Let $k^{\prime}$ be the residue field of $K^{\prime}$. As discussed in Section 4.18, we have $\left(\overline{\mathfrak{X}}^{\prime}\right)_{\text {can }}^{w}=\overline{\mathfrak{X}}_{\text {can }}^{w} \otimes_{k} k^{\prime}$ and $\operatorname{in}_{w}\left(X^{\prime}\right)=\operatorname{in}_{w}(X) \otimes_{k} k^{\prime}$, so the first assertion follows from the fact that $\overline{\mathfrak{X}}_{\text {can }}^{w} \otimes_{k} k^{\prime} \rightarrow \overline{\mathfrak{X}}_{\text {can }}^{w}$ induces a bijection on irreducible components. Let $\overline{\mathfrak{C}}$ be an irreducible component of $\overline{\mathfrak{X}}_{\text {can }}^{w}$, and let $\overline{\mathfrak{D}}$ be its image in $\overline{\mathfrak{X}}^{w}$. Then $[\overline{\mathfrak{C}}: \overline{\mathfrak{D}}]=\left[\overline{\mathfrak{C}} \otimes_{k} k^{\prime}: \overline{\mathfrak{D}} \otimes_{k} k^{\prime}\right]$, so relative multiplicities are preserved as well.

Later we will relate $m_{\text {rel }}(x)$ to other geometrically-defined notions of multiplicity; see Proposition 4.31 and Theorem 5.8. For the moment we relate relative multiplicities to tropical multiplicities, defined as follows.

Definition 4.23. Let $X \subset \mathbf{T}$ be a closed subscheme, and let $w \in \operatorname{Trop}(X)$. If $w \in N_{G}$, then the tropical multiplicity of $X$ at $w$ is defined to be

$$
m_{\operatorname{Trop}}(w)=\sum_{\bar{C} \subset \operatorname{in}_{w}(X)} \operatorname{mult}_{\operatorname{in}_{w}(X)}(\bar{C}),
$$

where the sum is taken over all irreducible components $\bar{C}$ of $\operatorname{in}_{w}(X)$. If $w \notin N_{G}$, then let $K^{\prime}$ be an algebraically closed complete valued field extension of $K$ such that $w \in N_{G^{\prime}}$, where $G^{\prime}$ is the 


\section{BAKer, S. PAyne And J. Rabinoff}

value group of $K^{\prime}$. Let $X^{\prime}=X \otimes_{K} K^{\prime}$. We define $m_{\operatorname{Trop}}(w)$ to be the tropical multiplicity of $w$ relative to trop: $\left(X^{\prime}\right)^{\text {an }} \rightarrow \operatorname{Trop}(X)$.

The fact that $m_{\text {Trop }}(w)$ is independent of the choice of $K^{\prime}$ is proved in [OP13, Remark A.5]. It is also one of the consequences of the following proposition.

Proposition 4.24. Let $X \subset \mathbf{T}$ be a reduced and equidimensional closed subscheme, and let $w \in \operatorname{Trop}(X)$. Then

$$
m_{\text {Trop }}(w)=\sum_{x \in \operatorname{trop}^{-1}(w)} m_{\text {rel }}(x) .
$$

Proof. We immediately reduce to the case where $w \in N_{G}$ by extending the ground field if necessary. By definition we have

$$
\sum_{x \in \operatorname{trop}^{-1}(w)} m_{\mathrm{rel}}(x)=\sum_{\overline{\mathfrak{C}} \subset \overline{\mathfrak{X}}_{\text {can }}^{w}}[\overline{\mathfrak{C}}: \operatorname{im}(\overline{\mathfrak{C}})]
$$

where the sum is taken over all irreducible components $\overline{\mathfrak{C}}$ of $\overline{\mathfrak{X}}_{\text {can }}^{w}$; the image $\operatorname{im}(\overline{\mathfrak{C}})$ of $\overline{\mathfrak{C}}$ in $\operatorname{in}_{w}(X)$ is an irreducible component by Corollary 3.11. Also by definition,

$$
m_{\text {Trop }}(w)=\sum_{\bar{C} \subset \operatorname{in}_{w}(X)} \operatorname{mult}_{\operatorname{in}_{w}(X)}(\bar{C}),
$$

where the sum is taken over all irreducible components $\bar{C}$ of $\operatorname{in}_{w}(X)$. By Proposition 3.25, for every irreducible component $\bar{C}$ of $\operatorname{in}_{w}(X)$ we have

$$
\operatorname{mult}_{\operatorname{in}_{w}(X)}(\bar{C})=\sum_{\overline{\mathfrak{C}} \rightarrow \bar{C}}[\overline{\mathfrak{C}}: \bar{C}],
$$

where the sum is taken over all irreducible components $\overline{\mathfrak{C}}$ of $\overline{\mathfrak{X}}_{\text {can }}^{w}$ mapping onto $\bar{C}$ (for any such $\overline{\mathfrak{C}}$ we have mult $\overline{\mathfrak{X}}_{\text {can }}^{w}(\overline{\mathfrak{C}})=1$ since $\overline{\mathfrak{X}}_{\text {can }}^{w}$ is reduced). Therefore

$$
m_{\text {Trop }}(w)=\sum_{\bar{C} \subset \operatorname{in}_{w}(X)} \operatorname{mult}_{\operatorname{in}_{w}(X)}(\bar{C})=\sum_{\bar{C} \subset \operatorname{in}_{w}(X)} \sum_{\overline{\mathfrak{C}} \rightarrow \bar{C}}[\overline{\mathfrak{C}}: \bar{C}]=\sum_{\overline{\mathfrak{C}} \subset \overline{\mathfrak{X}_{\text {can }}^{w}}}[\overline{\mathfrak{C}}: \operatorname{im}(\overline{\mathfrak{C}})] .
$$

EXAMPLE 4.25. In Example 2.6, we have $m_{\text {rel }}(\xi)=1$ for all $\xi \in \Gamma$ and $m_{\text {rel }}(\xi)=0$ for all $\xi \notin \Gamma$. This follows from Proposition 4.24 and the concrete description of the tropicalization map in Example 2.6, together with the observation that $m_{\text {Trop }}(0,0)=m_{\text {Trop }}(2,2)=1$ (since the initial degenerations $\operatorname{in}_{(0,0)}(E) \cong \operatorname{Spec} k\left[x, y, x^{-1}, y^{-1}\right] /\left(y^{2}-x^{3}-x^{2}\right)$ and $\operatorname{in}_{(2,2)}(E) \cong$ Spec $k\left[x, y, x^{-1}, y^{-1}\right] /\left(y^{2}-x^{2}-1\right)$ are both integral schemes over $\left.k\right)$. Note that $m_{\text {rel }}(\xi)>0$ for all $\xi \in \Gamma$ because $\xi$ is contained in the topological boundary of $\operatorname{trop}^{-1}(\operatorname{trop}(\xi))$ in $E^{\text {an }}$, hence in the Shilov boundary; see Section 3.4.

\subsection{Polyhedral structures on tropicalizations}

Let $W$ be a $G$-rational affine space in $N_{\mathbb{R}}$, and let $W_{0}$ be the linear space under $W$, so $W_{0}$ is spanned by $W_{0} \cap N$. Set $N^{\prime}=N /\left(W_{0} \cap N\right)$ and $M^{\prime}=W_{0}^{\perp} \cap M \subset M$, and let $\mathbf{T}^{\prime}$ be the torus $\operatorname{Spec}\left(K\left[M^{\prime}\right]\right)$. We call $\mathbf{T}^{\prime}$ the torus transverse to $W$. Let $w^{\prime} \in N_{G}^{\prime}$ be the image of any point of $W$. Then

$$
R\left[\left(\mathbf{T}^{\prime}\right)^{w^{\prime}}\right]=\left\{\sum_{u \in M^{\prime}} a_{u} x^{u} \in K\left[M^{\prime}\right]: \operatorname{val}\left(a_{u}\right)+\left\langle u, w^{\prime}\right\rangle \geqslant 0\right\},
$$

so for all $w \in N_{G} \cap W$ we have $R\left[\left(\mathbf{T}^{\prime}\right)^{w^{\prime}}\right] \subset R\left[\mathbf{T}^{w}\right]$. Hence we have a natural morphism $\pi_{w}: \overline{\mathbf{T}}^{w} \rightarrow$ $\left(\overline{\mathbf{T}}^{\prime}\right)^{w^{\prime}}$ for all $w \in N_{G} \cap W$. 


\section{NONARCHIMEDEAN GEOMETRY AND TROPICALIZATION}

Remark 4.27. Let $N^{\prime \prime}=\operatorname{ker}\left(N \rightarrow N^{\prime}\right)=W_{0} \cap N$, and let $M^{\prime \prime}=\operatorname{Hom}_{\mathbb{Z}}\left(N^{\prime \prime}, \mathbb{Z}\right)$, so we have exact sequences

$$
0 \longrightarrow N^{\prime \prime} \longrightarrow N \longrightarrow N^{\prime} \longrightarrow 0 \text { and } 0 \longrightarrow M^{\prime} \longrightarrow M \longrightarrow M^{\prime \prime} \longrightarrow 0
$$

inducing an exact sequence of tori

$$
0 \longrightarrow \mathbf{T}^{\prime \prime} \longrightarrow \mathbf{T} \longrightarrow \mathbf{T}^{\prime} \longrightarrow 0,
$$

where $\mathbf{T}^{\prime \prime}=\operatorname{Spec}\left(K\left[M^{\prime \prime}\right]\right)$. We call $\mathbf{T}^{\prime \prime}$ the torus parallel to $W$. Choosing a splitting of $N \rightarrow N^{\prime}$ splits all three exact sequences, and in particular furnishes an isomorphism $\mathbf{T} \cong \mathbf{T}^{\prime} \times \mathbf{T}^{\prime \prime}$. Let $w \in W \cap N_{G}$, and let $w^{\prime \prime}$ be its image in $N_{\mathbb{R}}^{\prime \prime}$. Then we have an isomorphism $\overline{\mathbf{T}}^{w} \cong\left(\overline{\mathbf{T}}^{\prime}\right)^{w^{\prime}} \times\left(\overline{\mathbf{T}}^{\prime \prime}\right)^{w^{\prime \prime}}$ under which $\pi_{w}$ corresponds to the projection onto the first factor.

TheOrem 4.28. Let $X \subset \mathbf{T}$ be an equidimensional subscheme of dimension $d$. The set $\operatorname{Trop}(X)$ admits a polyhedral complex structure of pure dimension $d$ with the following properties:

(i) The tropical multiplicities are constant along the relative interior of every maximal face.

(ii) Let $w$ be contained in the relative interior of a maximal face $\tau$ of $\operatorname{Trop}(X)$, let $W=\operatorname{span}(\tau)$, let $\mathbf{T}^{\prime}$ be the torus transverse to $W$, and let $\pi_{w}: \overline{\mathbf{T}}^{w} \rightarrow\left(\overline{\mathbf{T}}^{\prime}\right)^{w^{\prime}}$ be the natural map. Then $\operatorname{in}_{w}(X) \cong \pi_{w}^{-1}(\bar{Y})$ for some dimension 0 subscheme $\bar{Y}$ of $\left(\overline{\mathbf{T}}^{\prime}\right)^{w^{\prime}}$.

Proof. The first part is a basic result in tropical geometry; it is proved in [MS15, §3.3]. ${ }^{4}$ Let $\mathbf{T}^{\prime \prime} \subset \mathbf{T}$ be the torus parallel to $W$ and write $\overline{\mathbf{T}}^{\prime \prime}=\left(\overline{\mathbf{T}}^{\prime \prime}\right)^{0}$, so $\left(\overline{\mathbf{T}}^{\prime}\right)^{w^{\prime}}$ is the quotient of $\overline{\mathbf{T}}^{w}$ by $\overline{\mathbf{T}}^{\prime \prime}$. By [Spe05, Proposition 2.2.4], the initial degeneration $\operatorname{in}_{w}(X)$ is invariant under the action of $\overline{\mathbf{T}}^{\prime \prime}$. Therefore $\operatorname{in}_{w}(X)$ is the inverse image of a closed subscheme $\bar{Y}$ of $\left(\overline{\mathbf{T}}^{\prime}\right)^{w^{\prime}}$ (in fact $\bar{Y}$ is the quotient $\left.\operatorname{in}_{w}(X) / \overline{\mathbf{T}}^{\prime \prime} \subset\left(\overline{\mathbf{T}}^{\prime}\right)^{w^{\prime}}\right)$; counting dimensions, we see that $\operatorname{dim}(\bar{Y})=0$. See [MFK94, Theorem 1.1.1 and Amplification 1.1.3] for basic existence results about geometric quotients of affine schemes over a field by a free action of a reductive group.

In the situation of Theorem 4.28(ii), let $\mathbf{T}^{\prime \prime}$ be the torus parallel to $W$, and choose a splitting $\mathbf{T} \cong \mathbf{T}^{\prime} \times \mathbf{T}^{\prime \prime}$ as in Remark 4.27. Then $\operatorname{in}_{w}(X) \cong \bar{Y} \times\left(\overline{\mathbf{T}}^{\prime \prime}\right)^{w^{\prime \prime}}$.

\subsection{The tropical projection formula}

Let $X \subset \mathbf{T}$ be a reduced and equidimensional closed subscheme of dimension $d$, and let $P$ be an integral $G$-affine polytope contained in the relative interior of a maximal ( $d$-dimensional) face $\tau$ of a polyhedral complex decomposition of $\operatorname{Trop}(X)$ as in Theorem 4.28. Let $W$ be the affine span of $\tau$, let $\mathbf{T}^{\prime}$ be the torus transverse to $W$ (Section 4.26), let $\mathbf{T}^{\prime \prime}$ be the torus parallel to $W$ (Remark 4.27 ), and choose a splitting $\mathbf{T} \rightarrow \mathbf{T}^{\prime \prime}$. Note that $\operatorname{dim}\left(\mathbf{T}^{\prime \prime}\right)=d$. Let $P^{\prime \prime}$ be the image of $P$ in $N_{\mathbb{R}}^{\prime \prime}$, so $\mathscr{U}^{P^{\prime \prime}}$ is a polytopal domain in $\left(\mathbf{T}^{\prime \prime}\right)^{\text {an }}$. The map $\mathscr{U}^{P} \rightarrow \mathscr{U}^{P^{\prime \prime}}$ induces a morphism $\psi^{P}: \mathscr{X}^{P} \rightarrow \mathscr{U}^{P^{\prime \prime}}$.

ThEOREM 4.30. The morphism $\psi^{P}: \mathscr{X}^{P} \rightarrow \mathscr{U}^{P^{\prime \prime}}$ is finite, and every irreducible component of $\mathscr{X}^{P}$ surjects onto $\mathscr{U}^{P^{\prime \prime}}$.

Proof. For dimension reasons it suffices to show that $\psi^{P}$ is finite. Since $\mathscr{X}^{P}=\mathscr{M}(\mathcal{A})$ and $\mathscr{U}^{P}=\mathscr{M}\left(K\left\langle\mathscr{U}^{P^{\prime \prime}}\right\rangle\right)$ are both affinoid, by the rigid-analytic direct image theorem [BGR84,

\footnotetext{
${ }^{4}$ The proofs in $[\mathrm{MS} 15, \S 3.3]$ assume that there is a section to the valuation map val: $K^{\times} \rightarrow G$. Such a section always exists when $K$ is an algebraically closed nonarchimedean field; the following short proof was communicated to us by Speyer. If $G=\{0\}$, then there is nothing to prove. Otherwise, consider the short exact sequence $0 \rightarrow$ $U \rightarrow K^{\times} \rightarrow G \rightarrow 0$. Since $K$ is algebraically closed, the group $U$ is divisible. Thus $U$ is injective as a $\mathbb{Z}$-module, so $\operatorname{Ext}^{1}(A, U)=0$ and the valuation map splits.
} 


\section{BAKer, S. PAYne And J. RABinofF}

Theorem 9.6.3/1] it suffices to show that $\mathscr{X}^{P} \rightarrow \mathscr{U}^{P^{\prime \prime}}$ is proper in the sense of [BGR84, 9 9.6.2]. In fact we will show that $\mathscr{X}^{P} \Subset_{\mathscr{U}^{P^{\prime \prime}}} \mathscr{X}^{P}$, that is, that there exist affinoid generators $f_{1}, \ldots, f_{r}$ for $\mathcal{A}$ over $K\left\langle\mathscr{U}^{P^{\prime \prime}}\right\rangle$ such that $\left|f_{1}\right|_{\text {sup }}, \ldots,\left|f_{r}\right|_{\text {sup }}<1$.

Choosing bases for $N^{\prime}$ and $N^{\prime \prime}$, we obtain isomorphisms $N_{\mathbb{R}}^{\prime} \cong \mathbb{R}^{n^{\prime}}, N_{\mathbb{R}}^{\prime \prime} \cong \mathbb{R}^{d}$, and $N_{\mathbb{R}} \cong$ $\mathbb{R}^{n^{\prime}} \times \mathbb{R}^{d}$. Translating by an element of $\mathbf{T}(K)$, we may and do assume that $P \subset\{0\} \times N_{\mathbb{R}}^{\prime \prime}$ (so $\left.P=P^{\prime \prime}\right)$. For $\epsilon \in G$ with $\epsilon>0$ we let $I_{\epsilon} \subset N_{\mathbb{R}}^{\prime}$ be the cube $[-\epsilon, \epsilon]^{n^{\prime}}$, so $I_{\epsilon}$ is an integral $G$ affine polytope in $N_{\mathbb{R}}^{\prime}$, and $P_{\epsilon}:=I_{\epsilon} \times P^{\prime \prime}$ is an integral $G$-affine polytope in $N_{\mathbb{R}}=N_{\mathbb{R}}^{\prime} \times N_{\mathbb{R}}^{\prime \prime}$ containing $P$. Since $\tau$ is a maximal face, we have $P_{\epsilon} \cap \operatorname{Trop}(X)=P$ for small $\epsilon$; we fix such an $\epsilon$ as well as an element $e \in K$ with $\operatorname{val}(e)=\epsilon$. The polytopal subdomain $\mathscr{U}^{I_{\epsilon}} \subset\left(\mathbf{T}^{\prime}\right)^{\text {an }}$ is a product of annuli of inner radius $|e|$ and outer radius $|e|^{-1}$, so if $u_{1}, \ldots, u_{n^{\prime}}$ is a basis for $M^{\prime}$, then $\left\{e x^{ \pm u_{1}}, \ldots, e x^{ \pm u_{n^{\prime}}}\right\}$ is a set of affinoid generators for $K\left\langle\mathscr{U}^{I_{\epsilon}}\right\rangle$. Since $\mathscr{U}^{P_{\epsilon}}=\mathscr{U}^{I_{\epsilon}} \times_{K} \mathscr{U}^{P^{\prime \prime}}$, it follows that $\left\{e x^{ \pm u_{1}}, \ldots, e x^{ \pm u_{n^{\prime}}}\right\}$ is a set of affinoid generators for $K\left\langle\mathscr{U}^{P_{\epsilon}}\right\rangle$ over $K\left\langle\mathscr{U}^{P^{\prime \prime}}\right\rangle$. Since $P_{\epsilon} \cap \operatorname{Trop}(X)=P$, we have $X^{\text {an }} \cap \mathscr{U}^{P_{\epsilon}}=\mathscr{X}^{P}$, so $\left\{e x^{ \pm u_{1}}, \ldots, e x^{ \pm u_{n^{\prime}}}\right\}$ can be regarded as a set of affinoid generators for $\mathcal{A}$ over $K\left\langle\mathscr{U}^{P^{\prime \prime}}\right\rangle$. But by construction $\left|x^{u_{i}}(x)\right|=1$ for all $x \in \mathscr{X}^{P}$ and all $i=1, \ldots, n^{\prime}$, so $\left|e x^{u_{i}}(x)\right|=|e|<1$. This proves that $\psi^{P}$ is finite.

It follows from Theorem 4.30 and Remarks 3.18(i) and 3.22(i) that $\psi^{P}$ has a (pure) degree.

Proposition 4.31. In the situation of Section 4.29, let $\mathscr{Y} \subset \mathscr{X}^{P}$ be a union of connected components, and let $w \in P$. Then

$$
\left[\mathscr{Y}: \mathscr{U}^{P^{\prime \prime}}\right]=\sum_{x \in \mathscr{Y} \cap \operatorname{trop}^{-1}(w)} m_{\text {rel }}(x) .
$$

Proof. Extending the ground field if necessary, we assume $w \in N_{G}$. Let $w^{\prime \prime}$ be the image of $w$ in $N_{G}^{\prime \prime}$. Since $\mathscr{Y} \cap \mathscr{X}^{w} \rightarrow \mathscr{U}^{w^{\prime \prime}}$ is obtained by flat base change from $\mathscr{Y} \rightarrow \mathscr{U}^{P^{\prime \prime}}$, we may replace $P$ by $w$ and $P^{\prime \prime}$ by $w^{\prime \prime}$ to assume $\mathscr{Y} \subset \mathscr{X}^{w}$ (cf. Proposition 3.23(i)). Let $\mathfrak{Y}$ be the canonical model of $Y$. The canonical reduction $\overline{\mathfrak{Y}}$ of $\mathscr{Y}$ is a union of connected components of $\overline{\mathfrak{X}}_{\text {can }}^{w}$, so for $x \in \mathscr{Y}$ the relative multiplicity $m_{\text {rel }}(x)$ is nonzero if and only if $\operatorname{red}(x)$ is the generic point of an irreducible component $\overline{\mathfrak{C}}$ of $\overline{\mathfrak{Y}}$, in which case $m_{\text {rel }}(x)=[\overline{\mathfrak{C}}: \operatorname{im}(\overline{\mathfrak{C}})]$, where $\operatorname{im}(\overline{\mathfrak{C}})$ is the image of $\overline{\mathfrak{C}}$ in $\operatorname{in}_{w}(X)$. Noting that $\overline{\mathfrak{U}}^{w^{\prime \prime}}$ is an integral domain and $\overline{\mathfrak{Y}}$ is reduced, applying Proposition 3.25 to $\mathfrak{Y} \rightarrow \mathfrak{U}^{w^{\prime \prime}}$ yields

$$
\left[\mathscr{Y}: \mathscr{U}^{w^{\prime \prime}}\right]=\sum_{\mathfrak{C} \subset \overline{\mathfrak{Y}}}\left[\mathfrak{C}: \overline{\mathfrak{U}}^{w^{\prime \prime}}\right] .
$$

Since $\overline{\mathfrak{U}}^{w^{\prime \prime}}=\left(\overline{\mathbf{T}}^{\prime \prime}\right)^{w^{\prime \prime}}$ and $\operatorname{in}_{w}(X) \cong \bar{D} \times\left(\overline{\mathbf{T}}^{\prime \prime}\right)^{w^{\prime \prime}}$ for some dimension 0 scheme $\bar{D} \subset\left(\overline{\mathbf{T}}^{\prime}\right)^{w^{\prime}}$ (cf. Remark 4.27), the reduced space underlying any irreducible component of $\operatorname{in}_{w}(X)$ is isomorphic to $\left(\overline{\mathbf{T}}^{\prime \prime}\right)^{w^{\prime \prime}}$. Therefore $\left[\operatorname{im}(\overline{\mathfrak{C}}): \overline{\mathfrak{U}}^{w^{\prime \prime}}\right]=1$ for any irreducible component $\overline{\mathfrak{C}} \subset \overline{\mathfrak{Y}}$, so $\left[\overline{\mathfrak{C}}: \overline{\mathfrak{U}} w^{w^{\prime \prime}}\right]=[\overline{\mathfrak{C}}$ : $\operatorname{im}(\overline{\mathfrak{C}})]$ and the proposition follows.

Corollary 4.32 (Tropical projection formula). In the situation of Theorem 4.30, the degree of $\psi^{P}: \mathscr{X}^{P} \rightarrow \mathscr{U}^{P^{\prime \prime}}$ is equal to $m_{\text {Trop }}(w)$ for any $w \in P$.

Proof. Assuming $P=w$ and $P^{\prime \prime}=w^{\prime \prime}$ as in the proof of Proposition 4.31, the result follows immediately from Propositions 4.31 and 4.24 .

Remark 4.33. The tropical projection formula is an equality of the degree of the morphism $\mathscr{X}^{P} \rightarrow \mathscr{U}^{P^{\prime \prime}}$ (a morphism on the generic fiber) with the degree of a morphism $\overline{\mathfrak{X}}^{w} \rightarrow \overline{\mathfrak{U}}^{w^{\prime \prime}}$ (a morphism on the special fiber). It is conceptually very close to the projection formula as stated in Proposition 3.25, as indeed that is the main tool used in its proof; it is for this reason that we call it the tropical projection formula. 


\section{NONARCHIMEDEAN GEOMETRY AND TROPICALIZATION}

\section{The tropicalization of a nonarchimedean analytic curve}

In this section we freely use the definitions and notation from [BPR13]. In particular, for $a, b \in K^{\times}$we have $\mathbf{S}(a, b)=\left\{t \in \mathbf{G}_{m}^{\text {an }}:|a| \leqslant|t| \leqslant|b|\right\}$, a closed annulus; we also set $\mathbf{S}(a)=$ $\mathbf{S}(a, 1)$ and $\mathbf{S}(1)=\left\{t \in \mathbf{G}_{m}^{\text {an }}:|t|=1\right\}$, the closed annulus of modulus 0 .

\subsection{The setup}

Throughout this section $X$ denotes a smooth connected algebraic curve realized as a closed subscheme of a torus T, $\widehat{X}$ is the smooth completion of $X$, and $D=\widehat{X}(K) \backslash X(K)$ is the set of punctures. We will denote a choice of semistable vertex set for $X$ by $V$, and we let $\Sigma=\Sigma(X, V)$ be the associated skeleton. See [BPR13, §3].

If we choose a basis for $M$, then we obtain isomorphisms $N_{\mathbb{R}} \cong \mathbb{R}^{n}$ and $K[\mathbf{T}] \cong K\left[x_{1}^{ \pm 1}, \ldots\right.$, $\left.x_{n}^{ \pm 1}\right]$; if $f_{i} \in K[X]^{\times}$is the image of $x_{i}$, then

$$
\operatorname{trop}(\|\cdot\|)=\left(-\log \left\|f_{1}\right\|, \ldots,-\log \left\|f_{n}\right\|\right) .
$$

\subsection{Compatible polyhedral structures}

The tropicalization of $X$ is a polyhedral complex of pure dimension 1 in $N_{\mathbb{R}}$. We can regard $\operatorname{Trop}(X)$ as a dimension 1 abstract $G$-rational polyhedral complex where the metric on the edges is given by the lattice length, that is, the length in the direction of a primitive lattice vector in $N$.

Recall the following consequence of the slope formula [BPR13, Theorem 5.15].

Lemma 5.3. Let $e$ be an edge of $\Sigma$, and let $f \in K[X]^{\times}$. The map $\|\cdot\| \mapsto-\log \|f\|: X^{\text {an }} \rightarrow \mathbb{R}$ restricts to a $G$-affine linear function from $e$ to $\mathbb{R}$ with integer slope.

Since the $G$-rational points of an edge $e$ of $\Sigma$ are exactly the type 2 points of $X^{\text {an }}$ contained in $e$ (cf. [BPR13, $\S 3.12]$ ), it follows from (5.1.1) and the $G$-rationality of $-\log \|f\|$ as above that trop maps type 2 points into $N_{G}$.

Proposition 5.4. (i) The tropicalization map trop: $X^{\text {an }} \rightarrow \operatorname{Trop}(X)$ factors through the retraction $\tau_{\Sigma}: X^{\text {an }} \rightarrow \Sigma$.

(ii) We can choose $V$ and a polyhedral complex structure on $\operatorname{Trop}(X)$ as in Theorem 4.28 such that trop: $\Sigma \rightarrow \operatorname{Trop}(X)$ is a morphism of dimension 1 abstract $G$-rational polyhedral complexes.

Proof. The first part follows from (5.1.1) and the slope formula [BPR13, Theorem 5.15]. Let $e$ be an edge of $\Sigma$. It follows from Lemma 5.3 as applied to $f_{1}, \ldots, f_{n}$ that trop restricts to an expansion by an integer multiple with respect to the intrinsic metric on $e$ and the lattice length on its image. Hence there exist refinements of the polyhedral structures on $\Sigma$ and on $\operatorname{Trop}(X) \operatorname{such}$ that trop: $\Sigma \rightarrow \operatorname{Trop}(X)$ becomes a morphism of dimension 1 abstract $G$-rational polyhedral complexes. By [BPR13, Proposition 3.13(2)], any refinement of $\Sigma$ is also a skeleton of $X$.

From now on we assume that our skeleton $\Sigma$ of $X$ and our choice of polyhedral structure on $\operatorname{Trop}(X)$ are compatible in the sense of Proposition 5.4(ii). If $e \subset \Sigma$ is an interval contained in an edge, we let $\ell_{\mathrm{an}}(e)$ be its length with respect to the skeletal metric, and if $e^{\prime} \subset \operatorname{Trop}(X)$ is an interval contained in an edge, we let $\ell_{\text {Trop }}\left(e^{\prime}\right)$ be its lattice length. As a consequence of Proposition 5.4(ii), if $\operatorname{trop}(e)=e^{\prime}$, then $\ell_{\text {Trop }}\left(e^{\prime}\right)$ is an integer multiple of $\ell_{\text {an }}(e)$. 


\section{BAKer, S. PAyne And J. RabinofF}

Definition 5.5. Let $e \subset \Sigma$ be an edge, and let $e^{\prime} \subset \operatorname{Trop}(X)$ be its image. We define the expansion factor of $e$ to be the unique integer $m_{\text {rel }}(e) \in \mathbb{Z}_{\geqslant 0}$ such that

$$
\ell_{\text {Trop }}(\operatorname{trop}(\tilde{e}))=m_{\mathrm{rel}}(e) \cdot \ell_{\mathrm{an}}(\tilde{e})
$$

for any finite-length segment $\tilde{e}$ contained in $e$.

Remark 5.6. For $u \in M$ let $f_{u} \in K[X]^{\times}$be the image of the character $x^{u} \in K[M]$. Let $e$ be an edge of $\Sigma$, and let $s_{u} \in \mathbb{Z}_{\geqslant 0}$ be the absolute value of the slope of $-\log \left\|f_{u}\right\|$ on $e$. It follows easily from the definitions that $m_{\text {rel }}(e)=\operatorname{gcd}\left\{s_{u}: u \in M\right\}$. More concretely, let $u_{1}, \ldots, u_{n}$ be a basis for $M$, and let $f_{i} \in K[X]^{\times}$be the image of $x^{u_{i}}$, so $\operatorname{trop}(\|\cdot\|)=\left(-\log \left\|f_{1}\right\|, \ldots,-\log \left\|f_{n}\right\|\right)$. Let $s_{i} \in \mathbb{Z}_{\geqslant 0}$ be the absolute value of the slope of $-\log \left\|f_{i}\right\|$ on $e$. Then

$$
m_{\text {rel }}(e)=\operatorname{gcd}\left(s_{1}, \ldots, s_{n}\right) .
$$

5.7 We now come to one of the key results of this section. Let $e \subset \Sigma$ be a bounded edge, ${ }^{5}$ and assume that $e^{\prime}=\operatorname{trop}(e)$ is an edge of $\operatorname{Trop}(X)$ (as opposed to a vertex). The inverse image of the interior of $e^{\prime}$ under trop is a disjoint union of open annuli, one of whose skeleta is the interior of $e$. Hence if $x \in e$ is the unique point mapping to some $w \in \operatorname{relint}\left(e^{\prime}\right) \cap N_{G}$, then $\mathscr{Y}_{x}:=\tau_{\Sigma}^{-1}(x) \cong \mathbf{S}(1)$ is a connected component of $\mathscr{X}^{w}=\mathscr{U}^{w} \cap X^{\text {an }}$ (Definition 4.14). Let $W$ be the affine span of $e^{\prime}$, let $\mathbf{T}^{\prime}$ be the torus transverse to $W$ (Section 4.26), let $\mathbf{T}^{\prime \prime}$ be the torus parallel to $W$ (Remark 4.27), and choose a splitting $\mathbf{T} \rightarrow \mathbf{T}^{\prime \prime}$. We have a finite surjective morphism $\mathscr{X}^{w} \rightarrow \mathscr{U}^{w^{\prime \prime}}$ by Theorem 4.30, where $w^{\prime \prime}$ is the image of $w$ in $N_{G}^{\prime \prime}$.

For $y \in e$ the relative multiplicity $m_{\text {rel }}(y)$ was defined in Definition 4.21 .

THEOREM 5.8 (Compatibility of multiplicities). With the above notation,

$$
m_{\text {rel }}(e)=\left[\mathscr{Y}_{x}: \mathscr{U}^{w^{\prime \prime}}\right]=m_{\text {rel }}(x) .
$$

Moreover, $m_{\mathrm{rel}}(e)=m_{\mathrm{rel}}(y)$ for any $y$ in the interior of $e\left(\right.$ even if $\left.\operatorname{trop}(y) \notin N_{G}\right)$.

Proof. Let $P$ be a $G$-rational closed interval contained in the interior of $e^{\prime}$ and containing $w$. Then

$$
\mathscr{Y}:=\tau_{\Sigma}^{-1}\left(\operatorname{trop}^{-1}(P) \cap e\right) \cong \mathbf{S}(a, b)
$$

is a connected component of $\mathscr{X}^{P}$; it is a closed annulus of nonzero modulus with skeleton $\operatorname{trop}^{-1}(P) \cap e$. Let $P^{\prime \prime}$ be the image of $P$ in $N_{G}^{\prime \prime}$, so $\ell_{\text {Trop }}\left(P^{\prime \prime}\right)=\ell_{\text {Trop }}(P)$. By Theorem 4.30 the morphism $\mathscr{Y} \rightarrow \mathscr{U}^{P^{\prime \prime}}$ is finite and surjective, and $\left[\mathscr{Y}: \mathscr{U}^{P^{\prime \prime}}\right]=\left[\mathscr{Y}_{x}: \mathscr{U}^{w^{\prime \prime}}\right]$ by Proposition 3.23(i). The torus $\mathbf{T}^{\prime \prime}$ is 1-dimensional, so $\mathscr{U}^{P^{\prime \prime}}=\operatorname{trop}^{-1}\left(P^{\prime \prime}\right)$ is an annulus and $P^{\prime \prime}$ is by definition the skeleton of $\mathscr{U}^{P^{\prime \prime}}$. We have

$$
m_{\text {rel }}(e) \cdot \ell_{\text {an }}\left(\operatorname{trop}^{-1}(P) \cap e\right)=\ell_{\text {Trop }}(P)=\ell_{\text {Trop }}\left(P^{\prime \prime}\right)=\left[\mathscr{Y}: \mathscr{U}^{P^{\prime \prime}}\right] \cdot \ell_{\text {an }}\left(\operatorname{trop}^{-1}(P) \cap e\right),
$$

where the final equality is by [BPR13, Corollary 2.6]. Since $x$ is the unique Shilov boundary point of $\mathscr{Y}_{x} \cong \mathbf{S}(1)$, the equality $\left[\mathscr{Y}_{x}: \mathscr{U}^{w^{\prime \prime}}\right]=m_{\text {rel }}(x)$ is a consequence of Proposition 4.31 .

By a standard argument involving extension of the ground field (cf. Lemma 4.22 and Section 4.18), the second statement follows from the first.

\footnotetext{
${ }^{5}$ If $e$ is an infinite ray, we can compute $m_{\text {rel }}(e)$ by refining the polyhedral structure on $\Sigma$ so that $m_{\text {rel }}(e)$ coincides with $m_{\text {rel }}(\tilde{e})$ for some bounded edge $\tilde{e}$ in the refinement. Thus we can assume without loss of generality in Theorem 5.8 that $e$ is bounded.
} 


\section{NONARCHIMEDEAN GEOMETRY AND TROPICALIZATION}

Corollary 5.9. Fix an edge $e^{\prime}$ of $\operatorname{Trop}(X)$, and let $e_{1}, \ldots, e_{r}$ be the edges of $\Sigma$ mapping homeomorphically onto $e^{\prime}$. Then

$$
m_{\text {Trop }}\left(e^{\prime}\right)=\sum_{i=1}^{r} m_{\text {rel }}\left(e_{i}\right) .
$$

Proof. This follows immediately from Theorem 5.8 and Proposition 4.24.

Remark 5.10. With the notation in Corollary 5.9, let $w$ be a $G$-rational point contained in the relative interior of $e^{\prime}$. The affinoid space $\operatorname{trop}^{-1}(w)$ is isomorphic to $\coprod_{i=1}^{r} \mathbf{S}(1)$ as in Section 5.7. Since the canonical reduction of $\mathbf{S}(1)$ is isomorphic to $\mathbf{G}_{m}$, the integer $r$ is equal to the number of irreducible components in the canonical reduction of $\mathscr{X}^{w}$.

Corollary 5.11. If $e^{\prime}$ is an edge of $\operatorname{Trop}(X)$ and $m_{\text {Trop }}\left(e^{\prime}\right)=1$, then there is a unique edge $e$ in $\Sigma$ such that trop maps $e$ homeomorphically and isometrically onto $e^{\prime}$. The edge $e$ is in fact the unique geodesic segment (or ray) in $X^{\text {an }}$ which is mapped homeomorphically by trop onto $e^{\prime}$.

Corollary 5.12. Let $x \in \mathbf{H}\left(X^{\text {an }}\right)$. Then $m_{\text {rel }}(x)>0$ if and only if $x$ belongs to an edge of $\Sigma$ mapping homeomorphically onto its image via trop.

Proof. Suppose that $x$ is contained in an edge $e \subset \Sigma$ mapping homeomorphically onto its image $e^{\prime}=\operatorname{trop}(e)$. If $x$ is in the interior of $e$, then $m_{\text {rel }}(x)=m_{\text {rel }}(e)>0$ by Theorem 5.8. Otherwise $w=\operatorname{trop}(x) \in N_{G}$, and $x$ is contained in the limit boundary $\partial_{\lim } \mathscr{X}^{w}$ of $\mathscr{X}^{w}=\operatorname{trop}^{-1}(w)$ because it is a limit of points of $e$ which are not contained in $\mathscr{X}^{w}$. Since $\partial_{\text {lim }} \mathscr{X}^{w}$ is the Shilov boundary of $\mathscr{X}^{w}$, by definition we have $m_{\text {rel }}(x)>0$.

Now suppose that $x$ is not contained in an edge of $\Sigma$ mapping homeomorphically onto its image. If $x$ has type 4 , then $m_{\text {rel }}(x)=0$ by definition. Otherwise by [BPR13, Corollary 5.1] we can enlarge $\Sigma$ if necessary to assume $x \in \Sigma$. Recall that every edge of $\Sigma$ maps homeomorphically onto its image or is crushed to a vertex of $\operatorname{Trop}(X)$. By hypothesis all edges containing $x$ are crushed to a vertex of $\operatorname{Trop}(X)$, so $w=\operatorname{trop}(x) \in N_{G}$. Hence there is an open neighborhood $U$ of $x$ in $\Sigma$ contained in $\mathscr{X}^{w}$. Then $\tau_{\Sigma}^{-1}(U)$ is a neighborhood of $x$ in $X^{\text {an }}$ contained in $\mathscr{X}^{w}$, so $x \notin \partial \mathscr{X}^{w}$, and hence $m_{\text {rel }}(x)=0$.

\subsection{Slopes as orders of vanishing}

The slope formula [BPR13, Theorem 5.15] provides a useful interpretation of the quantities $s_{i}$ appearing in Remark 5.6 in terms of orders of vanishing. Assume that $V$ is a strongly semistable vertex set $[\mathrm{BPR} 13, \S 3]$ of $\widehat{X}$ (in addition to being a semistable vertex set of $X$ ). Let $\mathfrak{X}$ be the strongly semistable formal model of $\widehat{X}$ associated with $V$, let $x \in V$, and let $\overline{\mathfrak{C}}$ be the irreducible component of $\overline{\mathfrak{X}}$ whose generic point is $\operatorname{red}(x)$. Let $e$ be an edge of $\Sigma$ adjacent to $x$, and let $\xi \in \overline{\mathfrak{C}}(k)$ be the reduction of the interior of $e$. The slope formula [BPR13, Theorem 5.15] says that if $f$ is a nonzero rational function on $\widehat{X}$, then the slope $s$ of $-\log |f|$ along $e$ (in the direction away from $x)$ is equal to $\operatorname{ord}_{\xi}\left(\widetilde{f}_{x}\right)$. One can use this fact to give a simple proof of the well-known balancing formula for tropical curves.

Theorem 5.14 (Balancing formula for tropical curves). Let $w$ be a vertex of $\operatorname{Trop}(X)$, and let $\vec{v}_{1}, \ldots, \vec{v}_{t}$ be the primitive integer tangent directions at $w$ corresponding to the various edges $e_{1}^{\prime}, \ldots, e_{t}^{\prime}$ incident to $w$. Then $\sum_{j=1}^{t} m_{\text {Trop }}\left(e_{j}^{\prime}\right) \vec{v}_{j}=0$.

Proof. We use the setup of Section 5.13. Let $f_{1}, \ldots, f_{n} \in K[X]^{\times}$be the coordinate functions as in Remark 5.6, and let $F_{i}=-\log \left|f_{i}\right|$. Let $x \in \Sigma \cap \operatorname{trop}^{-1}(w)$ be a vertex. By the slope 


\section{BAKer, S. PAyne And J. RabinofF}

formula [BPR13, Theorem 5.15] we have $0=\sum_{v \in T_{x}} d_{v} F_{i}(x)$ for each $i=1, \ldots, n$. Since we are assuming that $V$ is a strongly semistable vertex set, each tangent direction $v \in T_{x}$ along which some $F_{i}$ has nonzero slope is represented by a unique edge $e_{v}=\left[x, y_{v}\right]$ of $\Sigma$ adjoining $x$, and $d_{v} F_{i}(x)$ is just the slope of $-\log \left|f_{i}\right|$ along $e_{v}$. If $\operatorname{trop}\left(e_{v}\right)=\{w\}$, then $d_{v} F_{i}(x)=0$ for all $i$, and otherwise

$$
d_{v} F_{i}(x)=\frac{\log \left|f_{i}(x)\right|-\log \left|f_{i}\left(y_{v}\right)\right|}{\ell_{\mathrm{an}}\left(e_{v}\right)}=m_{\mathrm{rel}}\left(e_{v}\right) \frac{\log \left|f_{i}(x)\right|-\log \left|f_{i}\left(y_{v}\right)\right|}{\ell_{\operatorname{Trop}}\left(\operatorname{trop}\left(e_{v}\right)\right)} .
$$

By Corollary 5.9, for each $i$ we have

$$
\sum_{j=1}^{t} m_{\operatorname{Trop}}\left(e_{j}^{\prime}\right)\left(\vec{v}_{j}\right)_{i}=\sum_{j=1}^{t}\left(\sum_{e=[x, y] \stackrel{\sim}{\longrightarrow} e_{j}^{\prime}} m_{\text {rel }}(e)\right) \frac{\log \left|f_{i}(x)\right|-\log \left|f_{i}(y)\right|}{\ell_{\operatorname{Trop}}\left(e_{j}^{\prime}\right)}=\sum_{x \mapsto w} \sum_{v \in T_{x}} d_{v} F_{i}(x)=0,
$$

which implies the result.

\subsection{Faithful representations}

If $Y_{\Delta}$ is a proper toric variety with dense torus $\mathbf{T}$, then $X \hookrightarrow \mathbf{T}$ extends in a unique way to a morphism $\iota: \widehat{X} \rightarrow Y_{\Delta}$, which is a closed immersion for suitable $Y_{\Delta}$. The intersection $\widehat{X}^{\text {an }} \cap$ $\left(Y_{\Delta}^{\mathrm{an}} \backslash \mathbf{T}^{\mathrm{an}}\right)$ is the finite set of type 1 points $D=\widehat{X}^{\text {an }} \backslash X^{\mathrm{an}}$. We write $\operatorname{trop}(\iota): \widehat{X}^{\text {an }} \rightarrow N_{\mathbb{R}}(\Delta)$ for the induced tropicalization map, and we set $\operatorname{Trop}(\widehat{X}, \iota)=\operatorname{trop}(\iota)\left(\widehat{X}^{\text {an }}\right) \subset N_{\mathbb{R}}(\Delta)$.

5.15.1 Let $Y_{\Delta}$ and $Y_{\Delta^{\prime}}$ be toric varieties with dense tori $\mathbf{T}$ and $\mathbf{T}^{\prime}$, respectively, and let $\iota: \widehat{X} \hookrightarrow$ $Y_{\Delta}$ and $\iota^{\prime}: \widehat{X} \hookrightarrow Y_{\Delta^{\prime}}$ be closed immersions whose images meet the dense torus. We say that $\iota^{\prime}$ dominates $\iota$ and we write $\iota^{\prime} \geqslant \iota$ provided that there exists a morphism $\psi: Y_{\Delta^{\prime}} \rightarrow Y_{\Delta}$ of toric varieties such that $\psi \circ \iota^{\prime}=\iota$. In this case we have an induced morphism $\operatorname{Trop}(\psi): \operatorname{Trop}\left(\widehat{X}, \iota^{\prime}\right) \rightarrow$ $\operatorname{Trop}(\widehat{X}, \iota)$ making the triangle

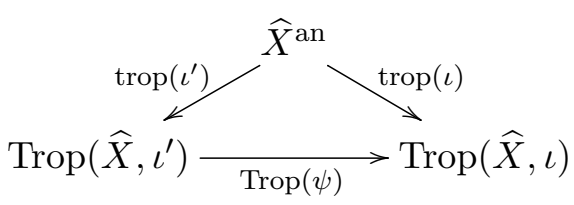

commute. Since $\operatorname{trop}(\iota)$ and $\operatorname{trop}\left(\iota^{\prime}\right)$ are surjective, the map $\operatorname{Trop}(\psi)$ is independent of the choice of $\psi$, so the set of 'tropicalizations of toric embeddings' is a filtered inverse system.

5.15.2 By a finite subgraph of $\widehat{X}^{\text {an }}$ we mean a connected compact subgraph of a skeleton of $\widehat{X}$. Any finite union of geodesic segments in $\mathbf{H}_{\circ}\left(\widehat{X}^{\text {an }}\right)$ is contained in a skeleton by [BPR13, Corollary 5.10], so we can equivalently define a finite subgraph of $\widehat{X}^{\text {an }}$ to be an isometric embedding of a finite connected metric graph $\Gamma$ into $\mathbf{H}_{\circ}\left(\widehat{X}^{\text {an }}\right)$. Let $\widehat{X} \hookrightarrow Y_{\Delta}$ be a closed immersion into a toric variety with dense torus $\mathbf{T}$ such that $\widehat{X} \cap \mathbf{T} \neq \emptyset$. We say that a finite subgraph $\Gamma$ of $\widehat{X}^{\text {an }}$ is faithfully represented by trop: $\widehat{X}^{\text {an }} \rightarrow N_{\mathbb{R}}(\Delta)$ if trop maps $\Gamma$ homeomorphically and isometrically onto its image $\Gamma^{\prime}$ (which is contained in $N_{\mathbb{R}}$ ). We say that trop is faithful if it faithfully represents a skeleton $\Sigma$ of $\widehat{X}$.

Remark 5.16. When considering a closed connected subset $\Gamma$ of $\mathbf{H}\left(X^{\text {an }}\right)$ or $\Gamma^{\prime}$ of $\operatorname{Trop}(X)$, we will always implicitly endow it with the shortest-path metric. In general this is not the same as the metric on $\Gamma$ (respectively, $\left.\Gamma^{\prime}\right)$ induced by the (shortest-path) metric on $\mathbf{H}\left(X^{\text {an }}\right)$ (respectively, $\operatorname{Trop}(X))$. With this convention, $\Gamma$ (respectively, $\Gamma^{\prime}$ ) is a length space in the sense of [Pap05, 


\section{NONARCHIMEDEAN GEOMETRY AND TROPICALIZATION}

Definition 2.1.2], so any homeomorphism $\Gamma \rightarrow \Gamma^{\prime}$ which is a local isometry is automatically an isometry by Corollary 3.4.6 of [Pap05]. This will be used several times in what follows.

The following result shows that if $\Gamma$ is faithfully represented by a given tropicalization, then it is also faithfully represented by all 'larger' tropicalizations.

Lemma 5.17. Let $\iota: \widehat{X} \hookrightarrow Y_{\Delta}$ and $\iota^{\prime}: \widehat{X} \hookrightarrow Y_{\Delta^{\prime}}$ be closed immersions of $\widehat{X}$ into toric varieties whose images meet the dense torus and which satisfy $\iota^{\prime} \geqslant \iota$. If a finite subgraph $\Gamma$ of $\widehat{X}^{\text {an }}$ is faithfully represented by $\operatorname{trop}(\iota): \widehat{X}^{\text {an }} \rightarrow N_{\mathbb{R}}(\Delta)$, then $\Gamma$ is faithfully represented by $\operatorname{trop}\left(\iota^{\prime}\right): \widehat{X}^{\text {an }} \rightarrow N_{\mathbb{R}}\left(\Delta^{\prime}\right)$.

Proof. Without loss of generality, we may replace $\widehat{X}$ by $X=\widehat{X} \backslash D$ and assume that $Y_{\Delta^{\prime}}$ and $Y_{\Delta}$ are tori with $\iota=\left(f_{1}, \ldots, f_{n}\right)$ and $\iota^{\prime}=\left(f_{1}, \ldots, f_{m}\right)$ for some $m \geqslant n$. The result is now clear from (5.6.1).

We will show in Theorem 5.20 that any finite subgraph of $\widehat{X}^{\text {an }}$ is faithfully represented by some tropicalization. First we need two lemmas.

Lemma 5.18. Let $e$ be an edge of a skeleton $\Sigma$ of $\widehat{X}$ with distinct endpoints $x$ and $y$. There exists a nonzero meromorphic function $f$ on $\widehat{X}$ such that $F=-\log |f|$ has the following properties:

(i) We have $F \geqslant 0$ on $e$, and $F(x)=F(y)=0$.

(ii) There exist (not necessarily distinct) type 2 points $x^{\prime}$ and $y^{\prime}$ in the interior $e^{\circ}$ of e such that $\rho\left(x, x^{\prime}\right)=\rho\left(y, y^{\prime}\right)$, such that $F$ has slope \pm 1 on $\left[x, x^{\prime}\right]$ and $\left[y, y^{\prime}\right]$, and such that $F$ is constant on $\left[x^{\prime}, y^{\prime}\right]$, as shown in Figure 4.

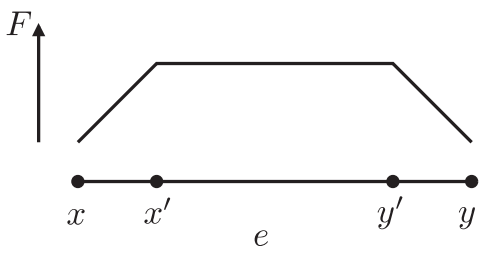

FiguRE 4 . The graph of the function $F=-\log |f|: e \rightarrow \mathbb{R}_{\geqslant 0}$ constructed in Lemma 5.18.

Proof. Without loss of generality, we may assume $\Sigma=\Sigma(\widehat{X}, V(\mathfrak{X}))$ for a strongly semistable formal model $\mathfrak{X}$ of $\widehat{X}$ [BPR13, § 4]. For each irreducible component $\overline{\mathfrak{C}}_{\nu}$ of $\overline{\mathfrak{X}}$, a simple argument using the Riemann-Roch theorem allows us to choose a rational function $\widetilde{f}_{\nu}$ on $\overline{\mathfrak{C}}_{\nu}$ which vanishes to order 1 at every singular point of $\overline{\mathfrak{X}}$ lying on $\overline{\mathfrak{C}}_{\nu}$. By [BL85, Corollary 3.8] there exists a nonzero rational function $f$ on $\widehat{X}$ whose poles all reduce to smooth points of $\overline{\mathfrak{X}}$ and which induces the rational function $\widetilde{f}_{\nu}$ on each irreducible component $\overline{\mathfrak{C}}_{\nu}$ of $\overline{\mathfrak{X}}$ (the gluing condition from loc. cit. is trivial in this situation). The function $f$ constructed in the proof of loc. cit. is defined on an affinoid domain $U$ of $\widehat{X}^{\text {an }}$ containing $x$ and $y$, and $\widetilde{f}_{x}$ and $\widetilde{f}_{y}$ are the restrictions of the residue of $f$ in the canonical reduction of $U$. Therefore we have $|f(x)|=|f(y)|=1$. Since $\{x, y\}$ is the Shilov boundary of $\tau_{\Sigma}^{-1}(e)$, this proves part (i).

By [BPR13, Theorem 5.15(3)] the outgoing slope of $F$ at $x$ or $y$ in the direction of $e$ is 1 . Let $\xi$ be the singular point of $\overline{\mathfrak{X}}$ whose formal fiber is $\tau_{\Sigma}^{-1}\left(e^{\circ}\right)$. Since $f$ has no poles on the formal fibers above singular points, $f$ restricts to an analytic function on the open annulus $\tau_{\Sigma}^{-1}\left(e^{\circ}\right)$. Part (ii) now follows from part (i) and [BPR13, Proposition 2.10(1)]. 


\section{BAKer, S. PAYne And J. RABinoff}

Lemma 5.19. Let $A \subset \widehat{X}^{\text {an }}$ be an affinoid domain isomorphic to a closed annulus $\mathbf{S}(a)$ with nonzero modulus. There exists a nonzero meromorphic function $f$ on $\widehat{X}$ such that $F=-\log |f|$ is linear with slope \pm 1 on $\Sigma(A)$.

Proof. Choose an identification of $A$ with $\mathbf{S}(a)=\mathscr{M}\left(K\left\langle a t^{-1}, t\right\rangle\right)$. By [FM86, Théorème 4, $\S 2.4$ ] the ring of meromorphic functions on $\widehat{X}$ which are regular on $A$ is dense in $K\left\langle a t^{-1}, t\right\rangle$. Hence there exists a meromorphic function $f$ on $\widehat{X}$ such that $f \in K\left\langle a t^{-1}, t\right\rangle$ and $|f-t|_{\text {sup }}<1$. It follows from [BPR13, Proposition 2.2] that $f$ is also a parameter for the annulus $A$, so $-\log |f|$ is linear with slope \pm 1 on $\Sigma(A)$.

THEOREM 5.20. If $\Gamma$ is any finite subgraph of $\widehat{X}^{\text {an }}$, then there is a closed immersion $\widehat{X} \hookrightarrow Y_{\Delta}$ of $\widehat{X}$ into a quasiprojective toric variety $Y_{\Delta}$ such that trop: $\widehat{X}^{\text {an }} \rightarrow N_{\mathbb{R}}(\Delta)$ faithfully represents $\Gamma$. In particular, there exists a faithful tropicalization.

Proof. Since $\Gamma$ is by definition contained in a skeleton $\Sigma$, we may assume $\Gamma=\Sigma$. Taking a refinement of $\Sigma$ if necessary, we assume without loss of generality that $\Sigma$ does not have any loop edges. We claim that after possibly refining $\Sigma$ further, for each edge $e \subset \Sigma$ there exists a nonzero meromorphic function $f$ on $\widehat{X}$ such that $\log |f|$ has slope \pm 1 on $e$.

Let $e=[x, y]$ be an edge of $\Sigma$, and let $f$ and $x^{\prime}, y^{\prime} \in e^{\circ}$ be as in Lemma 5.18. Then $\left[x, x^{\prime}\right]$ and $\left[y^{\prime}, y\right]$ are edges in a refinement of $\Sigma$, and $\log |f|$ has slope \pm 1 on $\left[x, x^{\prime}\right]$ and $\left[y^{\prime}, y\right]$. If $x^{\prime}=y^{\prime}$, then we are done with $e$; otherwise we let $e^{\prime}=\left[x^{\prime}, y^{\prime}\right]$. By construction $e^{\prime} \subset e^{\circ}$, so $\tau_{\Sigma}^{-1}\left(e^{\prime}\right)$ is a closed annulus of nonzero modulus, and we may apply Lemma 5.19 to find $f^{\prime}$ such that $\log \left|f^{\prime}\right|$ has slope \pm 1 on $e^{\prime}$. This proves the claim.

By (5.6.1), if $\Phi=\left\{f_{1}, \ldots, f_{r}\right\}$ is any collection of meromorphic functions on $X$ such that (i) for each edge $e$ of $\Sigma$ there is an $i$ such that $\log \left|f_{i}\right|$ has slope \pm 1 on $e$, and (ii) $\phi=\left(f_{1}, \ldots, f_{r}\right)$ induces a closed immersion of a dense open subscheme $X$ of $\widehat{X}$ into a torus $\mathbf{T} \cong \mathbf{G}_{m}^{r}$, then trop $\circ \phi$ maps each edge of $\Sigma$ isometrically onto its image. Since $\phi$ extends to a closed immersion $\widehat{X} \hookrightarrow Y_{\Delta}$ into a suitable compactification $Y_{\Delta}$ of $\mathbf{T}$, it only remains to show that we can enlarge $\Phi$ so that $\left.\phi\right|_{\Sigma}$ is injective; then trop $\circ \phi$ maps $\Sigma$ isometrically onto its image by Remark 5.16.

Let $e$ be an edge of $\Sigma$. Since $\Sigma$ has at least two edges, the proof of [BPR13, Theorem 4.11] (specifically case 1 in (4.15.1) of [BPR13]) shows that $\tau_{\Sigma}^{-1}(e)$ is an affinoid domain in $\widehat{X}^{\text {an }}$. By [FM86, Théorème $1, \S 1.4]$, there is a meromorphic function $f$ on $\widehat{X}$ such that $\tau_{\Sigma}^{-1}(e)=\{x \in$ $\left.\widehat{X}^{\text {an }}:|f(x)| \leqslant 1\right\}$. Adding such an $f$ to $\Phi$ for every edge $e$, we may assume that trop $\circ \phi$ is injective on $\Sigma \backslash V$, that is, that if $\operatorname{trop}(\phi(x))=\operatorname{trop}(\phi(y))$ for $x, y \in \Sigma$, then $x$ and $y$ are vertices. By the definition of $\widehat{X}^{\text {an }}$, if $x, y \in \widehat{X}^{\text {an }}$ are distinct points, then there exists a meromorphic function $f$ on $\widehat{X}^{\text {an }}$ such that $|f(x)| \neq|f(y)|$. Adding such $f$ to $\Phi$ for every pair of vertices yields a faithful tropicalization.

We obtain the following theorem as a consequence.

THEOREM 5.21. Let $\Gamma$ be a finite subgraph of $\widehat{X}$. Then there exists a quasiprojective toric embedding $\iota: \widehat{X} \hookrightarrow Y_{\Delta}$ such that for every quasiprojective toric embedding $\iota^{\prime}: \widehat{X} \hookrightarrow Y_{\Delta^{\prime}}$ with $\iota^{\prime} \geqslant \iota$, the tropicalization map $\operatorname{trop}\left(\iota^{\prime}\right): \widehat{X}^{\text {an }} \rightarrow N_{\mathbb{R}}\left(\Delta^{\prime}\right)$ maps $\Gamma$ homeomorphically and isometrically onto its image.

Proof. By Theorem 5.20, there exists a closed embedding $\iota$ such that trop $(\iota)$ maps $\Gamma$ homeomorphically and isometrically onto its image. By Lemma 5.17, the same property holds for any closed embedding $\iota^{\prime} \geqslant \iota$. 


\section{NONARCHIMEDEAN GEOMETRY AND TROPICALIZATION}

As mentioned in the introduction, Theorem 5.21 can be interpreted colloquially as saying that the homeomorphism in Theorem 1.2 is an isometry.

With a little more work, we obtain the following strengthening of Theorem 5.21 in which the finite metric graph $\Gamma$ is replaced by an arbitrary skeleton of $X$ (which is no longer required to be compact or of finite length).

Theorem 5.22. Let $\Sigma$ be any skeleton of $X$. Then there exists a quasiprojective toric embedding $\iota: X \hookrightarrow Y_{\Delta}$ such that for every quasiprojective toric embedding $\iota^{\prime}: X \hookrightarrow Y_{\Delta^{\prime}}$ with $\iota^{\prime} \geqslant \iota$, the tropicalization map trop $\left(\iota^{\prime}\right): \widehat{X}^{\text {an }} \rightarrow N_{\mathbb{R}}\left(\Delta^{\prime}\right)$ maps $\Sigma$ homeomorphically and isometrically onto its image.

Proof. Using Lemma 5.17, it suffices to prove that there exists a closed embedding $\iota$ such that $\operatorname{trop}(\iota)$ maps $\Sigma$ homeomorphically and isometrically onto its image.

For each point $p \in D=\widehat{X} \backslash X$, choose a pair of relatively prime integers $m_{1}(p), m_{2}(p)$ greater than $2 g$, where $g$ is the genus of $\widehat{X}$. By the Riemann-Roch theorem, there are rational functions $f_{1}^{(p)}$ and $f_{2}^{(p)}$ on $\widehat{X}$ such that $f_{i}^{(p)}$ has a pole of exact order $m_{i}(p)$ at $p$ and no other poles for $i=1,2$. Let $U_{p}$ be an (analytic) open neighborhood of $p$ on which $f_{1}^{(p)}$ and $f_{2}^{(p)}$ have no zeros, and let $U$ be the union of $U_{p}$ for all $p \in D$.

Let $\Gamma=\Sigma \backslash(\Sigma \cap U)$. Then $\Gamma$ is a finite subgraph of $X$, hence by Theorem 5.20 there exists a closed embedding $\iota_{0}$ such that $\operatorname{trop}\left(\iota_{0}\right)$ maps $\Gamma$ homeomorphically and isometrically onto its image. We can choose the $U_{p}$ such that the complement $\Sigma \backslash \Gamma$ consists of finitely many open infinite rays $r_{p}$, one for each point $p \in D$. By the slope formula, the absolute value of the slope of $\log \left|f_{i}^{(p)}\right|$ along $r_{p}$ is $m_{i}(p)$. Since $\operatorname{gcd}\left(m_{1}(p), m_{2}(p)\right)=1$ for all $p \in D$, if we enhance the embedding $\iota_{0}$ to a larger embedding $\iota$ by adding the coordinate functions $f_{1}^{(p)}$ and $f_{2}^{(p)}$ for all $p \in D, \operatorname{trop}(\iota)$ has multiplicity 1 along each ray $r_{p}$ by Remark 5.6. By Lemma 5.17, $\operatorname{trop}(\iota)$ also has multiplicity 1 at every edge of $\Gamma$. It follows easily (as in the proof of Theorem 5.20) that $\operatorname{trop}(\iota)$ maps $\Sigma$ homeomorphically and isometrically onto its image, as desired.

\subsection{Certifying faithfulness}

It is useful to be able to certify that a given tropicalization map is faithful using only 'tropical' computations.

THEOREM 5.24. Let $\Gamma^{\prime}$ be a compact connected subset of $\operatorname{Trop}(X)$, and suppose that $m_{\text {Trop }}(w)=1$ for all $w \in \Gamma^{\prime} \cap N_{G}$. Then there is a unique closed subset $\Gamma \subset \mathbf{H}_{\circ}\left(X^{\text {an }}\right)$ mapping homeomorphically onto $\Gamma^{\prime}$, and this homeomorphism is an isometry.

Proof. Since $m_{\text {Trop }}$ is constant along the interior of each edge of $\operatorname{Trop}(X)$ by Theorem 4.28(i) (for points not contained in $N_{G}$ this is proved by a standard ground-field extension argument) and $\Gamma^{\prime}$ is a finite union of closed intervals, we have $m_{\text {Trop }}(w)=1$ for all $w \in \Gamma^{\prime}$. By Proposition 4.24 , for each $w \in \Gamma^{\prime}$ there is a unique point $x=x_{w} \in \mathbf{H}_{\circ}\left(X^{\text {an }}\right)$ such that $\operatorname{trop}(x)=w$ and $m_{\text {rel }}(x)>0$. Let $\Gamma=\left\{x_{w}: w \in \Gamma^{\prime}\right\}$. The natural continuous map trop: $\Gamma \rightarrow \Gamma^{\prime}$ is bijective. It follows from Corollaries 5.11 and 5.12 that $\Gamma$ is also a finite union of closed intervals, hence compact. Thus trop: $\Gamma \rightarrow \Gamma^{\prime}$, being a continuous bijection between compact Hausdorff spaces, is a homeomorphism. By Corollary 5.11 this homeomorphism is an isometry.

As for the uniqueness of $\Gamma$, let $\widetilde{\Gamma}$ be any closed subset of $\mathbf{H}_{\circ}\left(X^{\text {an }}\right)$ mapping homeomorphically onto $\Gamma^{\prime}$. Fix $w \in \Gamma^{\prime}$, and let $x$ be the point in $\widetilde{\Gamma}$ with $\operatorname{trop}(x)=w$. Since $x$ belongs to a closed 


\section{BAKer, S. PAYne And J. RABinoff}

segment of $X^{\text {an }}$ mapping homeomorphically onto its image via trop (namely the inverse image in $\widetilde{\Gamma}$ of an edge in $\operatorname{Trop}(X)$ containing $w$ ), it follows from Corollary 5.12 that $m_{\text {rel }}(x)>0$. Hence $x=x_{w}$, so $\widetilde{\Gamma}=\Gamma$.

In order to apply Theorem 5.24, it is useful to know that one can sometimes determine the multiplicity at a point $w \in \operatorname{Trop}(X) \cap N_{G}$ just from the local structure of $\operatorname{Trop}(X)$ at $w$, that is, from the combinatorics of $\operatorname{Star}(w)$. Recall that if $\vec{v}_{1}, \ldots, \vec{v}_{r}$ are the primitive generators of the edge directions in $\operatorname{Trop}(X)$ at $w$, and $a_{i}$ is the tropical multiplicity of the edge corresponding to $\vec{v}_{i}$, then the balancing condition says that $a_{1} \vec{v}_{1}+\cdots+a_{r} \vec{v}_{r}=0$. Now, if $Z_{1}, \ldots, Z_{s}$ are the irreducible components of $\operatorname{in}_{w}(X)$, then the tropicalization of each $Z_{j}$ (as a subscheme of the torus torsor $\overline{\mathbf{T}}^{w}$ over the trivially valued field $k$ ) is a union of rays spanned by a subset of $\left\{\vec{v}_{1}, \ldots, \vec{v}_{r}\right\}$. If $b_{i j}$ is the multiplicity of the ray spanned by $\vec{v}_{i}$ in $\operatorname{Trop}\left(Z_{j}\right)$ and $m_{i}$ is the multiplicity of $Z_{i}$ in $\operatorname{in}_{w}(X)$, then the balancing condition implies $b_{1 j} \vec{v}_{1}+\cdots+b_{r j} \vec{v}_{r}=0$, and we also have $m_{1} b_{i 1}+\cdots+m_{s} b_{i s}=a_{i}$, since $\operatorname{Trop}\left(\operatorname{in}_{w}(X)\right)=\operatorname{Star}_{w}(\operatorname{Trop}(X))$ by [Spe14, Proposition 10.1].

Theorem 5.25. Let $w \in \operatorname{Trop}(X) \cap N_{G}$. If $\operatorname{Trop}(X)$ is trivalent at $w$ and one of the edges adjacent to $w$ has multiplicity 1 , then $w$ has multiplicity 1.

Proof. Let $\vec{v}_{1}, \vec{v}_{2}, \vec{v}_{3}$ be the primitive generators of the edge directions in $\operatorname{Trop}(X)$ at $w$, and let $a_{i}$ be the multiplicity of the edge in direction $\vec{v}_{i}$. The linear span $\left\langle\vec{v}_{1}, \vec{v}_{2}, \vec{v}_{3}\right\rangle$ is 2-dimensional, since the $\vec{v}_{i}$ are distinct and satisfy the balancing condition, so any relation among them is a scalar multiple of the relation $a_{1} \vec{v}_{1}+a_{2} \vec{v}_{2}+a_{3} \vec{v}_{3}=0$.

Let $Z$ be an irreducible component of $\operatorname{in}_{w}(X)$, and let $b_{i}$ be the multiplicity of the ray spanned by $\vec{v}_{i}$ in $\operatorname{Trop}(Z)$. Then $b_{i}$ is a nonnegative integer bounded above by $a_{i}$ and $b_{1} \vec{v}_{1}+b_{2} \vec{v}_{2}+b_{3} \vec{v}_{3}=0$. This relation must be a scalar multiple of the relations given by the $a_{i}$, so there is a positive rational number $\lambda \leqslant 1$ such that $b_{i}=\lambda a_{i}$ for all $i$. If some $a_{i}$ is 1 , then $\lambda$ must also be 1 and $b_{i}=a_{i}$ for all $i$. Since $a_{i}$ is the sum of the multiplicities of the ray spanned by $\vec{v}_{i}$ in the tropicalizations of the components of $\operatorname{in}_{w}(X)$, it follows that $\operatorname{in}_{w}(X)$ has no other components, and $w$ has multiplicity 1 .

Remark 5.26. Initial degenerations at interior points of an edge of multiplicity 1 are always smooth, since they are isomorphic to $\mathbf{G}_{m}$ by Theorem 4.28.

Remark 5.27. There are other natural combinatorial conditions which can guarantee multiplicity 1 at a point $w \in N_{G}$ of $\operatorname{Trop}(X)$ or smoothness of the corresponding initial degeneration. In the case of curves, for example, the argument above works more generally if $\operatorname{Trop}(X)$ is $r$-valent, the linear span of the edge directions at $w$ has dimension $r-1$, and the multiplicities of the edges at $w$ have no nontrivial common factor.

Combining the previous two results and the discussion of tropical hypersurfaces in Section 2.1, we obtain the following. Recall that a leaf in a graph is a vertex of valence 1 .

Corollary 5.28. Suppose $g(\widehat{X}) \geqslant 1$, and let $\Sigma$ be the minimal skeleton of $\widehat{X}^{\text {an }}$.

(i) If all vertices of $\operatorname{Trop}(X)$ are trivalent, all edges of $\operatorname{Trop}(X)$ have multiplicity 1 , the minimal skeleton $\Sigma$ has no leaves, ${ }^{6}$ and $\operatorname{dim} H_{1}(\Sigma, \mathbb{R})=\operatorname{dim} H_{1}(\operatorname{Trop}(X), \mathbb{R})$, then trop: $\Sigma \rightarrow$ $\operatorname{Trop}(X)$ is an isometry onto its image.

\footnotetext{
${ }^{6}$ In earlier versions of this paper, the skeleton $\Sigma$ was assumed to be bridgeless; however, the proof only used that $\Sigma$ has no leaves.
} 


\section{NONARCHIMEDEAN GEOMETRY AND TROPICALIZATION}

(ii) If $X \subset \mathbf{G}_{m}^{2}$ is defined by a polynomial $f \in K[x, y]$ whose Newton complex (see Remark 2.2) is a unimodular triangulation, then $\widehat{X}$ has totally degenerate reduction and trop: $\Sigma \rightarrow$ $\operatorname{Trop}(X)$ induces an isometry from $\Sigma$ onto its image.

Proof. Let $g^{\prime}=\operatorname{dim} H_{1}(\Sigma, \mathbb{R})=\operatorname{dim} H_{1}(\operatorname{Trop}(X), \mathbb{R})$. Choose a compact, connected subgraph $\Sigma^{\prime} \subset \operatorname{Trop}(X)$ with $g^{\prime}=\operatorname{dim} H_{1}\left(\Sigma^{\prime}, \mathbb{R}\right)$. By Theorem 5.25, all $w \in \operatorname{Trop}(X) \cap N_{G}$ have multiplicity 1 , so according to Theorem 5.24 , there is a (unique) finite subgraph $\widetilde{\Sigma}$ of $X^{\text {an }}$ mapping homeomorphically and isometrically onto $\Sigma^{\prime}$. Since $\Sigma$ is the minimal skeleton of $X^{\text {an }}$ and $\Sigma$ has no leaves, any subgraph of $X^{\text {an }}$ whose first homology has dimension at least $g^{\prime}$ must contain $\Sigma$. In particular, $\Sigma \subset \widetilde{\Sigma}$, so $\Sigma$ maps isometrically onto its image, which proves part (i).

We now prove part (ii). By Section 2.1, $\operatorname{Trop}(X)$ is trivalent with all edges of multiplicity 1. By Baker's theorem [Bak93] (see also [Bee09]), the genus $g(\widehat{X})$ is bounded by the number of internal vertices in the Newton polytope of $f$. Since the Newton complex is dual to the tropicalization, each internal vertex corresponds to a region in $\mathbb{R}^{2} \backslash \operatorname{Trop}(X)$; from this and the above, one sees that $g(\widehat{X}) \leqslant \operatorname{dim} H_{1}(\operatorname{Trop}(X), \mathbb{R})$. With $\Sigma^{\prime}$ as above, by Theorem 5.24 , there is a (unique) finite subgraph $\widetilde{\Sigma}$ of $X^{\text {an }}$ mapping homeomorphically onto $\Sigma^{\prime}$ via trop. Since $\operatorname{dim} H_{1}(\Sigma, \mathbb{R}) \geqslant$ $\operatorname{dim} H_{1}(\widetilde{\Sigma}, \mathbb{R}) \geqslant g(\widehat{X})$ in any case, it follows from the genus formula [BPR13, Remark 4.18] that $\operatorname{dim} H_{1}(\Sigma, \mathbb{R})=g(\widehat{X})$ and that $\widehat{X}$ has totally degenerate reduction. In particular, the minimal skeleton $\Sigma$ has no leaves. By part (i), trop: $\Sigma \rightarrow \operatorname{Trop}(X)$ is an isometry onto its image.

Corollary 5.28 will be important for applications to Tate curves in Section 6 .

EXAMPLE 5.29. As an example where the hypotheses of Corollary 5.28 are satisfied, consider the genus 3 curve $X=V(f)$ with

$$
\begin{aligned}
f= & t^{4}\left(x^{4}+y^{4}+z^{4}\right)+t^{2}\left(x^{3} y+x^{3} z+x y^{3}+x z^{3}+y^{3} z+y z^{3}\right) \\
& +t\left(x^{2} y^{2}+x^{2} z^{2}+y^{2} z^{2}\right)+x^{2} y z+x y^{2} z+x y z^{2} .
\end{aligned}
$$

See Figure 5.

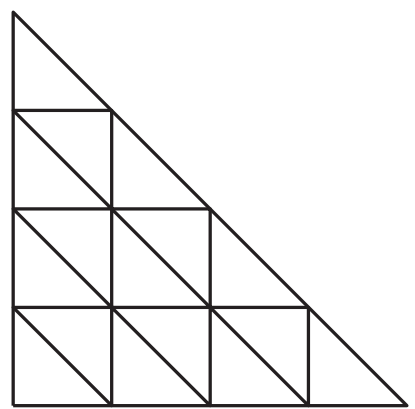

Newton complex

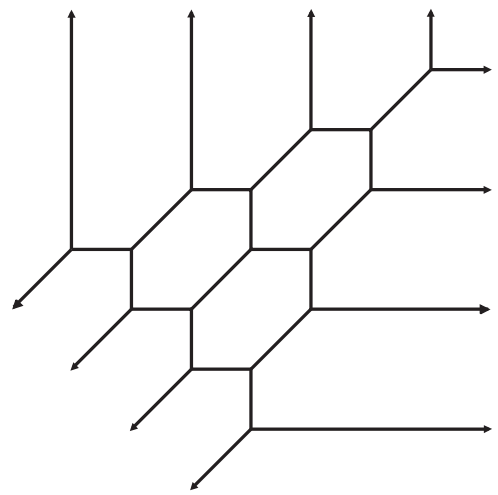

$\operatorname{Trop}(X)$

Figure 5. The Newton complex and tropicalization of the curve $X$ defined by the polynomial $f$ from Example 5.29. The tropicalization faithfully represents the minimal skeleton of $X^{\text {an }}$.

EXAMPLE 5.30. The following example shows that it is possible, even under the hypotheses of Theorem 5.24, for the tropicalization map to fail to be faithful. Let $K$ be the completion 


\section{BAKer, S. PAyne And J. RabinofF}

of $C\{\{t\}\}$, let $\widehat{X} \subset \mathbb{P}^{2}$ be defined by the equation

$$
(y-1)^{2}=(x-1)^{2}(y+1)+t \cdot x y
$$

over $\mathbb{C}\{\{t\}\}$, and let $X=\widehat{X} \cap \mathbf{G}_{m}^{2}$. The above equation degenerates to a nodal rational curve when $t=0$, the node being [1:1:0], so it defines a (not strongly) semistable algebraic integral model $\mathcal{X}$ of $\widehat{X}$-in fact, $\mathcal{X}$ is a minimal stable model for $\widehat{X}$, and the associated semistable vertex set only contains one point, so it is minimal as well (see [BPR13, Corollary 4.23]). Therefore $\widehat{X}$ is an elliptic curve with bad reduction and $\operatorname{in}_{0}(X) \cong \overline{\mathcal{X}} \cap \mathbf{G}_{m, k}^{2}$ is reduced and irreducible. The points in the set of punctures

$$
D:=\widehat{X}(K) \backslash X(K)=\{[0: 0: 1],[0: 1: 0],[1: 0: 0],[0: 3: 1],[2: 0: 1]\}
$$

reduce to distinct smooth points of $\overline{\mathcal{X}}$, so if $\Sigma$ is the minimal skeleton of $\widehat{X}^{\text {an }}$ and $\tau_{\Sigma}: \widehat{X}^{\text {an }} \rightarrow \Sigma$ is the retraction, then $\tau_{\Sigma}(x)$ reduces to the generic point of $\overline{\mathcal{X}}$ for all $x \in D$. Therefore the minimal skeleton $\Gamma$ of $X$ and the tropicalization of $X$ are as shown in Figure 6. We see that Trop $(X)$ is contractible and has everywhere multiplicity 1 but the image of the section does not contain the loop in $X^{\text {an }}$ (the loop is contracted to the origin). In particular, trop is not faithful despite the fact that all points in $\operatorname{Trop}(X)$ have multiplicity 1.

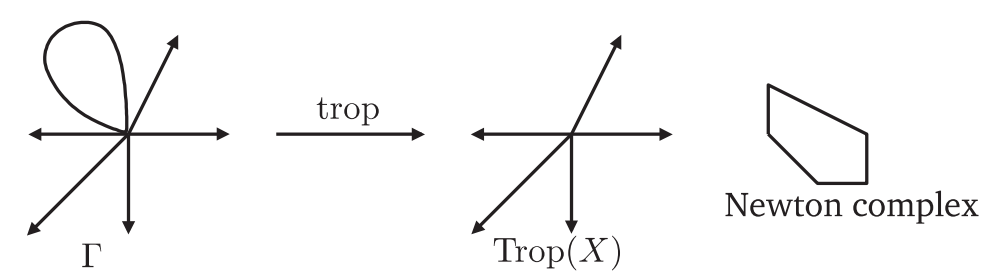

Figure 6. The skeleton, tropicalization, and Newton complex of the curve $X$ from Example 5.30. One sees from the Newton complex that the initial degenerations are all of multiplicity 1 away from 0 , and $\operatorname{in}_{0}(X)$ is a rational nodal curve. However, the tropicalization crushes the loop in $X^{\text {an }}$ to the origin.

\section{Elliptic curves}

Let $\widehat{E} / K$ be an elliptic curve. If $\widehat{E}$ has good reduction, then the minimal skeleton $\Sigma$ of $\widehat{E}^{\text {an }}$ is a point, while if $\widehat{E}$ has multiplicative reduction, then the minimal skeleton $\Sigma$ of $\widehat{E}^{\text {an }}$ is homeomorphic to a circle of length $-\operatorname{val}\left(j_{\widehat{E}}\right)=\operatorname{val}\left(q_{\widehat{E}}\right)$, where $\widehat{E}^{\text {an }} \cong \mathbf{G}_{m}^{\text {an }} / q_{\widehat{E}}^{\mathbb{Z}}$ is the Tate uniformization of $\widehat{E}$ (see [BPR13, Remark 4.24]). In this section, we use our results on nonarchimedean analytic curves and their tropicalizations to prove some new results (and reinterpret some old results) about tropicalizations of elliptic curves.

\subsection{Faithful tropicalization of elliptic curves}

As noted in [KMM08, KMM09], a curve in $\mathbf{G}_{m}^{2}$ given by a Weierstrass equation $y^{2}=x^{3}+a x^{2}+$ $b x+c$ cannot have a cycle in its tropicalization, because the Newton complex of a Weierstrass equation does not have an interior vertex. Thus Weierstrass equations are always 'bad' from the point of view of tropical geometry. On the other hand, the following result shows that there do always exist 'good' plane embeddings of elliptic curves with multiplicative reduction. 


\section{NONARCHIMEDEAN GEOMETRY AND TROPICALIZATION}

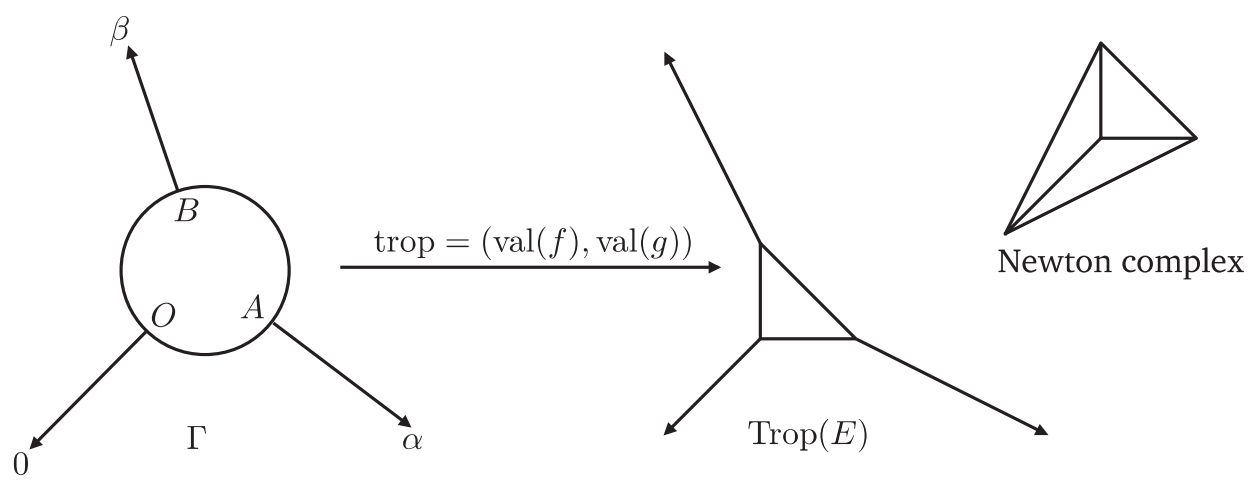

Figure 7. The skeleton $\Gamma$ of $E$, the tropicalization of $E$, and the Newton complex of the equation $a x^{2} y+b x y^{2}+c x y z=d z^{3}$ defining $E$, where $E$ is as in the proof of Theorem 6.2. The minimal skeleton $\Sigma$ of $\widehat{E}$ is the circle contained in $\Gamma$. The tropicalization faithfully represents $\Sigma$, so $\ell_{\text {Trop }}(\operatorname{Trop}(\Sigma))=\ell_{\text {an }}(\Sigma)$.

THEOREM 6.2. Let $\widehat{E} / K$ be an elliptic curve with multiplicative reduction. Then there is a closed embedding of $\widehat{E}$ in $\mathbb{P}^{2}$, given by a projective plane equation of the form $a x^{2} y+b x y^{2}+c x y z=d z^{3}$, such that (letting $E$ be the open affine subset of $\widehat{E}$ mapping into the torus $\mathbf{G}_{m}^{2}$ ) Trop $(E)$ is a trivalent graph, every point of $\operatorname{Trop}(E)$ has a smooth and irreducible initial degeneration (hence tropical multiplicity 1 ), and the minimal skeleton of $\widehat{E}$ is faithfully represented by the tropicalization map. In particular, $\operatorname{Trop}(E)$ contains a cycle of length $-\operatorname{val}\left(j_{\widehat{E}}\right)$.

Proof. Let $q=q_{\widehat{E}}$ be the Tate parameter, so that $\widehat{E}^{\text {an }} \cong \mathbf{G}_{m}^{\text {an }} / q^{\mathbb{Z}}$. Choose a cube root $q^{1 / 3} \in$ $K^{\times}$of $q$, and let $\alpha, \beta \in \widehat{E}(K)$ correspond under the Tate isomorphism to the classes of $q^{1 / 3}$ and $\left(q^{1 / 3}\right)^{2}$, respectively. Recall that a divisor $D=\sum a_{P}(P)$ on an elliptic curve is principal if and only if $\sum a_{P}=0$ and $\sum a_{P} P=0$ in the group law on the curve. In particular, there exist rational functions $f$ and $g$ on $\widehat{E}$ (unique up to multiplication by a nonzero constant) such that $\operatorname{div}(f)=2(\alpha)-(\beta)-(0)$ and $\operatorname{div}(g)=2(\beta)-(\alpha)-(0)$. Let $\psi: \widehat{E} \rightarrow \mathbb{P}^{2}$ be the morphism associated with the rational map $[f: g: 1]$. Since $1, f, g$ form a basis for $L(D)$ with $D=(\alpha)+(\beta)+(0)$ and $D$ is very ample [Har77, Corollary IV.3.2(b)], we see that $\psi$ is a closed immersion.

Let $\Gamma$ be the minimal skeleton of $E$, that is, the smallest closed connected subset of $\widehat{E}^{\text {an }}$ containing the skeleton $\Sigma$ of $\widehat{E}^{\text {an }}$ and the three points $\alpha, \beta, 0$, with those three points removed. Recall that $\Sigma$ is isometric to a circle of circumference $\ell_{\mathrm{an}}(\Sigma)=-\operatorname{val}\left(j_{\widehat{E}}\right)=\operatorname{val}(q)$. The natural map

$$
K^{\times} \rightarrow \widehat{E}(K) \hookrightarrow \widehat{E}^{\text {an }} \rightarrow \Sigma \stackrel{\sim}{\longrightarrow} \mathbb{R} / \ell \mathbb{Z}
$$

is given by $z \mapsto[\operatorname{val}(z)]$; in particular, if $\tau_{\Sigma}: \widehat{E}^{\text {an }} \rightarrow \Sigma$ denotes the canonical retraction, we have $\tau_{\Sigma}(0)=[0], \tau_{\Sigma}(\alpha)=\left[\frac{1}{3} \operatorname{val}(q)\right]$, and $\tau_{\Sigma}(\beta)=\left[\frac{2}{3} \operatorname{val}(q)\right]$. Thus $\Gamma$ is a circle with an infinite ray emanating from each of three equally spaced points $O=\tau_{\Sigma}(0), A=\tau_{\Sigma}(\alpha), B=\tau_{\Sigma}(\beta)$ along the circle (see Figure 7). The tropicalization map trop: $E=\widehat{E}^{\text {an }} \backslash\{0, \alpha, \beta\} \rightarrow \mathbb{R}^{2}$ corresponding to the embedding $E \hookrightarrow \mathbf{G}_{m}^{2}$ given by $(f, g)$ factors through the retraction onto $\Gamma$.

The map trop: $\Gamma \rightarrow \mathbb{R}^{2}$ can be determined (up to an additive translation) using the slope formula [BPR13, Theorem 5.15] by solving an elementary graph potential problem. The result is as follows. The function $\operatorname{val}(f)=-\log |f|$ has slope -1 along the ray from $O$ to 0 , slope 2 along the ray from $A$ to $\alpha$, and slope -1 along the ray from $B$ to $\beta$. On $\Sigma$, it has slope 1 along the segment from $O$ to $A$, slope -1 along the segment from $A$ to $B$, and slope 0 along the segment 


\section{BAKer, S. PAYne And J. RABinoff}

from $B$ to $O$. Similarly, the function $\operatorname{val}(g)=-\log |g|$ has slope -1 along the ray from $O$ to 0 , slope -1 along the ray from $A$ to $\alpha$, and slope 2 along the ray from $B$ to $\beta$. On $\Sigma$, it has slope 0 along the segment from $O$ to $A$, slope 1 along the segment from $A$ to $B$, and slope -1 along the segment from $B$ to $O$. Thus (up to a translation on $\mathbb{R}^{2}$ ) Trop $(E)$ is a trivalent graph consisting of a triangle with an infinite ray emanating from each of the vertices, as in Figure 7.

Since the expansion factor along every edge of $\Gamma$ is equal to 1 by (5.6.1), it follows from Corollary 5.9 that the tropical multiplicity of every edge of $\operatorname{Trop}(E)$ is 1 . By Theorem 5.25, the multiplicity at every vertex of $\operatorname{Trop}(E)$ is 1 as well, and in fact the initial degenerations are smooth and irreducible since the Newton complex is unimodular (see Figure 7). Since the expansion factor is 1 along every edge of $\Sigma$ and trop $\left.\right|_{\Sigma}$ is a homeomorphism, it follows that $\Sigma$ is faithfully represented. The bounded edges of $\operatorname{Trop}(E)$ form a triangle each of whose sides has lattice length $\operatorname{val}(q) / 3$.

The only thing which remains to be proved is that $\psi(\widehat{E}) \subset \mathbb{P}^{2}$ is cut out by an equation of the form indicated in the statement of the theorem. This follows from the Riemann-Roch theorem: the functions $1, f g, f^{2} g, f g^{2}$ all belong to the 3 -dimensional vector space $L(3(0))$ and hence there is a nonzero linear relation between them. (This argument is similar to [Har77, Proposition IV.4.6].)

We can also use our theorems to give more conceptual proofs of many of the results from [KMM08, KMM09]. For example, we have the following theorem which was proved in [KMM09] by a brute-force computation.

TheOrem 6.3. Let $E \subset \mathbf{G}_{m}^{2}$ be the intersection of an elliptic curve $\widehat{E} \subset \mathbb{P}^{2}$ with $\mathbf{G}_{m}^{2}$. Assume that (i) $\operatorname{Trop}(E)$ contains a cycle $C$, (ii) all edges of $\operatorname{Trop}(E)$ have multiplicity 1, and (iii) $\operatorname{Trop}(E)$ is trivalent. Then $\ell_{\text {Trop }}(C)=-\operatorname{val}\left(j_{\widehat{E}}\right)$.

Proof. This follows immediately from Corollary 5.28(i).

Remark 6.4. Conditions (i)-(iii) from Theorem 6.3 are automatically satisfied if the Newton complex of the defining polynomial for $E$ is a unimodular triangulation with a vertex lying in the interior of the Newton polygon, as in Figure 7. Varying the valuation of the coefficient corresponding to the interior vertex while keeping all other coefficients fixed gives a natural map from an annulus in $\mathbf{G}_{m}$ to the $j$-line, which is finite and flat onto an annulus in the $j$-line, by [BPR13, Proposition 2.2(2)]. In particular, given a tropical plane curve dual to such a Newton complex and an elliptic curve $E$ with $j$-invariant equal to minus the length of the loop, there is an embedding of $E$ into a toric variety such that the tropicalization of the intersection with $\mathbf{G}_{m}^{2}$ is faithful and equal to the given tropical curve. See [CS13] for explicit constructions of such embeddings for tropical curves of 'honeycomb normal form', including an algorithm for finding the honeycomb form of an elliptic curve, paramaterization by theta functions, the tropicalization of the inflection points, and relations to the group law.

Remark 6.5. A different (but related) conceptual explanation for Theorem 6.3 is given in [HK12, Proposition 7.7].

Let us say that a closed embedding of an elliptic curve $\widehat{E} / K$ in some toric variety is certifiably of genus 1 if $\operatorname{Trop}(E)$ satisfies conditions (i)-(iii) from Theorem 6.3. Note that the cycle $C$ in any such embedding satisfies $\ell_{\text {Trop }}(C)=-\operatorname{val}\left(j_{\widehat{E}}\right)$, by Theorem 6.3. Combining Theorems 6.2 and 6.3 , we obtain the following result.

COROLlary 6.6. An elliptic curve $\widehat{E} / K$ has multiplicative reduction if and only if it has a closed embedding in $\mathbb{P}^{2}$ which is certifiably of genus 1 . 


\section{NONARCHIMEDEAN GEOMETRY AND TROPICALIZATION}

\subsection{Speyer's well-spacedness condition}

In this section we explain how Speyer's well-spacedness condition [Spe14] follows from a more general result (possibly of independent interest) about the analytification of an elliptic curve $\widehat{E} / K$.

Let $\Sigma$ be the minimal skeleton of $\widehat{E}$. For $P, Q \in \widehat{E}(K)$, define $i(P, Q) \in \mathbb{R}_{\geqslant 0} \cup\{\infty\}$ as follows:

$$
i(P, Q)= \begin{cases}0 & \text { if } \tau_{\Sigma}(P) \neq \tau_{\Sigma}(Q), \\ \operatorname{dist}(P \vee Q, \Sigma) & \text { if } \tau_{\Sigma}(P)=\tau_{\Sigma}(Q),\end{cases}
$$

where $\tau_{\Sigma}: \widehat{E}^{\mathrm{an}} \rightarrow \Sigma$ is the retraction map, $P \vee Q$ is the first point where the geodesic paths from $P$ to $\Sigma$ and $Q$ to $\Sigma$ meet, and $\operatorname{dist}(x, \Sigma)$ is the distance (in the natural metric on $\mathbf{H}\left(\widehat{E}^{\text {an }}\right)$ ) from $x \in \widehat{E}^{\text {an }}$ to its retraction $\tau_{\Sigma}(x) \in \Sigma$. By convention we set $i(P, P)=+\infty$. Since translation by a point $P \in \widehat{E}(K)$ is an automorphism of $\widehat{E}$, it induces an isometry on $\mathbf{H}\left(\widehat{E}^{\text {an }}\right)$; therefore $i(P, Q)$ only depends on the difference $P-Q$ in $\widehat{E}(K)$; that is, $i(P, Q)=\iota(P-Q)$ with $\iota(R)=i(R, 0)$.

The following lemma shows that $\|P, Q\|:=\exp (-i(P, Q))$ is an ultrametric on $\widehat{E}(K)$.

Lemma 6.8. (i) For any points $P, Q, R \in \widehat{E}(K)$ we have $i(P, Q) \geqslant \min \{i(P, R), i(Q, R)\}$, with equality if $i(P, R) \neq i(Q, R)$.

(ii) If $m \in \mathbb{Z}$ is an integer such that $|m|=1$ in $K$, then $i(m P, m Q)=i(P, Q)$ for any $P, Q \in \widehat{E}(K)$ such that $i(P, Q)>0$.

Proof. We begin by proving part (i). If either $i(P, R)=0$ or $i(Q, R)=0$, then the inequality is trivial. Moreover, by translation invariance of $i$ we may assume $R=0$. So we are reduced to showing that if $\iota(P)>0$ and $\iota(Q)>0$, then $\iota(P-Q) \geqslant \min \{\iota(P), \iota(Q)\}$.

Let $\mathfrak{E}$ be the semistable formal model of $\widehat{E}$ corresponding to the semistable vertex set $\left\{\tau_{\Sigma}(0)\right\}$ (see [BPR13, Remark 4.24]); note that $\left.\Sigma=\Sigma\left(\widehat{E},\left\{\tau_{\Sigma}(0)\right\}\right)\right)$. Then $\overline{\mathfrak{E}}$ is a nodal rational curve, and the smooth locus $\overline{\mathfrak{E}^{\mathrm{sm}}}$ is a group scheme isomorphic to $\mathbf{G}_{m, k}$. The subset $\widehat{E}^{1}(K):=\{P \in \widehat{E}(K): \iota(P)>0\}$ is the formal fiber over the identity element of $\overline{E^{s m}}$, hence is a subgroup. In fact, $\widehat{E}^{1}(K)$ is isomorphic to the group $\mathfrak{m}=\{z \in K:|z|<1\}$ with the law of composition given by a 1-parameter formal group law $F$ over $R$ [Sil09, Proposition VII.2.1], and the restriction of $\iota$ to $\widehat{E}^{1}(K)$ corresponds to the valuation on $\mathfrak{m}$ under this identification. The desired inequality follows since a group law on $\mathfrak{m}$ given by a power series with coefficients in $R$ is obviously ultrametric.

In the situation of part (ii), as above we are reduced to showing that $\iota(m P)=\iota(P)$ when $\iota(P)>0$. This is true because $m$ is the coefficient of the linear term of the power series for multiplication by $m$ under $F$ [Sil09, Proposition IV.2.3], and all other terms have greater valuation.

Since $\iota(P)=\iota(-P)$, an equivalent formulation of Lemma 6.8(i) is that for any $P, Q \in \widehat{E}(K)$ we have $\iota(P+Q) \geqslant \min \{\iota(P), \iota(Q)\}$, with strict inequality if $\iota(P) \neq \iota(Q)$.

If $f$ is a nonconstant rational function on $\widehat{E}$, define $N_{f}$ to be the set of all $x \in \mathbf{H}\left(\widehat{E}^{\mathrm{an}}\right)$ such that $\log |f|$ is nonconstant in every open neighborhood of $x$. Equivalently, for $x \in \mathbf{H}\left(\widehat{E}^{\text {an }}\right)$ let $T_{x}(f)$ be the (finite) set of tangent directions at $x$ along which the derivative of $\log |f|$ is nonzero. Then $N_{f}$ is the set of all $x \in \widehat{E}^{\text {an }}$ such that $T_{x}(f) \neq \emptyset$. By [BPR13, Theorem 5.15(1,2)], $N_{f}$ is a union of finitely many edges of the minimal skeleton $\Gamma_{f}$ of the curve obtained from $\widehat{E}^{\text {an }}$ by removing all zeros and poles of $f$.

TheOREM 6.9. Suppose that $K$ has residue characteristic 0 . Let $f$ be a nonconstant rational function on $\widehat{E}$, and assume that there exists an $x \in N_{f} \operatorname{such}$ that $\operatorname{dist}(x, \Sigma)<\operatorname{dist}(y, \Sigma)$ for all $y \in N_{f}$ with $y \neq x$. Assume also $\Sigma \cap N_{f}=\emptyset$. Then $\left|T_{x}(f)\right| \geqslant 3$. 


\section{BAKer, S. PAyne And J. RabinofF}

In other words, either the minimum distance from $N_{f}$ to the skeleton is achieved at two distinct points, or else the minimum is achieved at a unique point at which $\log |f|$ has nonzero slope in at least three different tangent directions.

Proof. By the slope formula [BPR13, Theorem 5.15], the sum of the outgoing slopes of $\log |f|$ at $x$ is 0 , so $\left|T_{x}(f)\right| \geqslant 2$. For the sake of contradiction, assume $\left|T_{x}(f)\right|=2$ and write $T_{x}(f)=\left\{v, v^{\prime}\right\}$. Our hypotheses imply $x \notin \Sigma$ and that $\Sigma$ lies in a single connected component of $\widehat{E}^{\text {an }} \backslash\{x\}$.

Let $B(x, v)$ (respectively, $B\left(x, v^{\prime}\right)$ ) be the open set consisting of all $z \in \widehat{E}^{\text {an }}$ lying in the tangent direction $v$ (respectively, $v^{\prime}$ ), so that $B(x, v)$ and $B\left(x, v^{\prime}\right)$ are connected components of $\widehat{E}^{\text {an }} \backslash\{x\}$ which are disjoint from $\Sigma$. Let $D_{v}$ be the restriction of $\operatorname{div}(f)$ to $B(x, v)$, and let $D_{v^{\prime}}$ be the restriction of $\operatorname{div}(f)$ to $B\left(x, v^{\prime}\right)$. By the slope formula, we have $m=\operatorname{deg}\left(D_{v}\right)=-\operatorname{deg}\left(D_{v^{\prime}}\right)$ for some nonzero integer $m$. Without loss of generality, we may assume $m>0$. Let $\delta=\operatorname{dist}(x, \Sigma)>0$.

We claim that $\operatorname{div}(f)$ can be written as

$$
\operatorname{div}(f)=m((P)-(Q))+\sum_{j}\left(\left(A_{j}\right)-\left(B_{j}\right)\right)
$$

with $i(P, Q)=\delta$ and $i\left(A_{j}, B_{j}\right)>\delta$ for all $j$. Because we have assumed that $K$ has residue characteristic 0 , we have $|m|=1$, and therefore $i(m(P), m(Q))=i(P, Q)=\delta$ by Lemma 6.8(ii). Since $m P-m Q=\sum\left(B_{j}-A_{j}\right)$ in the group law on $\widehat{E}(K)$, we obtain a contradiction to Lemma 6.8(i).

To prove the claim, we use a trick due to Speyer. Suppose $D_{v}=\left(P_{1}\right)+\cdots+\left(P_{r}\right)-\left(Q_{1}\right)-$ $\cdots-\left(Q_{s}\right)$ and $D_{v^{\prime}}=\left(P_{1}^{\prime}\right)+\cdots+\left(P_{r^{\prime}}^{\prime}\right)-\left(Q_{1}^{\prime}\right)-\cdots-\left(Q_{s^{\prime}}^{\prime}\right)$ with $r-s=m$ and $s^{\prime}-r^{\prime}=m$. Then

$$
\begin{aligned}
D_{v}+D_{v^{\prime}}= & m\left(\left(P_{1}\right)-\left(Q_{1}^{\prime}\right)\right)+\sum_{j=1}^{s}\left(\left(P_{j}\right)-\left(Q_{j}\right)\right)+\sum_{j=s+1}^{r}\left(\left(P_{j}\right)-\left(P_{1}\right)\right) \\
& +\sum_{j=1}^{r^{\prime}}\left(\left(P_{j}^{\prime}\right)-\left(Q_{j}^{\prime}\right)\right)+\sum_{j=r^{\prime}+1}^{s^{\prime}}\left(\left(Q_{1}^{\prime}\right)-\left(Q_{j}^{\prime}\right)\right) .
\end{aligned}
$$

Note that $i\left(P_{1}, Q_{1}^{\prime}\right)=\delta$ but that $i\left(P_{j}, Q_{j}\right)>\delta$ for all $j=1, \ldots, s ; i\left(P_{j}, P_{1}\right)>\delta$ for all $j=$ $s+1, \ldots, r ; i\left(P_{j}^{\prime}, Q_{j}^{\prime}\right)>\delta$ for all $j=1, \ldots, r^{\prime}$; and $i\left(Q_{1}^{\prime}, Q_{j}^{\prime}\right)>\delta$ for all $j=r^{\prime}+1, \ldots, s^{\prime}$.

Let $C_{1}, \ldots, C_{t}$ be the connected components of $N_{f}$, labeled so that $C_{1}$ is the component containing $x$. By the slope formula [BPR13, Theorem 5.15], for each $j$ the restriction $D_{j}$ of $\operatorname{div}(f)$ to $C_{j}$ is a nonzero divisor of degree 0 and $\operatorname{div}(f)=\sum_{j} D_{j}$. Moreover, if $A, B \in \widehat{E}(K) \cap C_{j}$, then the unique geodesic paths from $A$ to $\Sigma$ and $B$ to $\Sigma$ must pass through the unique point $x_{j}$ of $C_{j}$ closest to $\Sigma$, so for $j \geqslant 2$ we have $i(A, B)>\delta$. The claim now follows since $D_{1}=D_{v}+D_{v^{\prime}}$ can be written as above, and by what we have just said we can write each $D_{j}$ for $j \geqslant 2$ as a sum of divisors of the form $(A)-(B)$ with $i(A, B)>\delta$.

In particular, we obtain the necessity of Speyer's well-spacedness condition for a genus 1 tropical curve to lift.

Corollary 6.10 (Speyer). Suppose that $K$ has residue characteristic 0. Let $E$ be a dense open subset of an elliptic curve $\widehat{E}$ over $K$ with multiplicative reduction and let $\psi: E \hookrightarrow \mathbf{T}$ be a closed embedding of $E$ in a torus T. Assume that (i) every vertex of $\operatorname{Trop}(E)$ is trivalent, (ii) every edge of $\operatorname{Trop}(E)$ has multiplicity 1, and (iii) $\operatorname{Trop}(E)$ contains a cycle $\Sigma^{\prime}$ which is contained in a hyperplane $H$. If $W_{H}$ denotes the closure in $N_{\mathbb{R}}$ of the set of points of $\operatorname{Trop}(E)$ not lying in $H$, then there is no single point of $W_{H}$ which is closest to $\Sigma^{\prime}$. 


\section{NONARCHIMEDEAN GEOMETRY AND TROPICALIZATION}

In other words, 'the minimum distance from points of $\operatorname{Trop}(E)$ not lying in $H$ to the cycle must be achieved twice'.

Proof. We may assume that $\psi: E \rightarrow \mathbf{T} \cong \mathbf{G}_{m}^{n}$ is given by $\left(f_{1}, \ldots, f_{n}\right)$ with $\log \left|f_{n}\right|$ equal to a constant $c$ on $\Sigma$ and that $H$ is the hyperplane $x_{n}=c$. Let $\Gamma$ be the minimal skeleton of $E$. By Corollary 5.28(i), we see that trop: $\Gamma \rightarrow \operatorname{Trop}(E)$ is an isometry. Since $N_{f_{n}} \subset \Gamma$, the result now follows from Theorem 6.9.

Remark 6.11. Katz [Kat12] and Nishinou [Nis09] have recently obtained other kinds of generalizations of Speyer's well-spacedness condition. Their generalized conditions apply to curves of higher genus.

\section{A generalization of the Sturmfels-Tevelev multiplicity formula}

As an illustration of the tools developed in this paper, we conclude with a generalization of the Sturmfels-Tevelev multiplicity formula [ST08, Theorem 1.1] to the nonconstant coefficient case (and also to nonsmooth points). A different proof is given in an appendix to [OP13].

Let $X \subset \mathbf{T}$ be a closed subvariety, that is, a reduced and irreducible closed subscheme. Let $\alpha: \mathbf{T} \rightarrow \mathbf{T}^{\prime}$ be a homomorphism of tori that induces a generically finite map of degree $\delta$ from $X$ to $X^{\prime}$, where $X^{\prime}$ is the closure of $\alpha(X)$. Then, set theoretically, $\operatorname{Trop}\left(X^{\prime}\right)$ is the image of $\operatorname{Trop}(X)$ under the induced linear map $A: N_{\mathbb{R}} \rightarrow N_{\mathbb{R}}^{\prime}$ [Tev07, Proposition 3]. The fundamental problem of tropical elimination theory is to determine the multiplicities on the maximal faces of $\operatorname{Trop}\left(X^{\prime}\right)$ from those on the maximal faces of $\operatorname{Trop}(X)$.

Theorem 7.1. Let $\alpha: \mathbf{T} \rightarrow \mathbf{T}^{\prime}$ be a homomorphism of algebraic tori over $K$, and let $X$ be a closed subvariety of $\mathbf{T}$. Let $X^{\prime}$ be the schematic image of $X$ in $\mathbf{T}^{\prime}$, let $f: X \rightarrow X^{\prime}$ be the restriction of $\alpha$ to $X$, and let $F=\operatorname{trop}(f): \operatorname{Trop}(X) \rightarrow \operatorname{Trop}\left(X^{\prime}\right)$ be the restriction of the linear map $A: N_{\mathbb{R}} \rightarrow N_{\mathbb{R}}^{\prime}$ induced by $\alpha$. Suppose that $f$ is generically finite of degree $\delta$. Then for any point $w^{\prime} \in \operatorname{Trop}\left(X^{\prime}\right) \cap N_{G}^{\prime}$ such that $\left|F^{-1}\left(w^{\prime}\right)\right|<\infty$, we have

$$
m_{\text {Trop }}\left(w^{\prime}\right)=\frac{1}{\delta} \sum_{w \in F^{-1}\left(w^{\prime}\right)} \sum_{\overline{\mathfrak{C}}^{\prime} \subset \overline{\mathfrak{X}}^{w}} \operatorname{mult}_{\overline{\mathfrak{X}}^{w}}(\overline{\mathfrak{C}})[\overline{\mathfrak{C}}: \operatorname{im}(\overline{\mathfrak{C}})],
$$

where the second sum runs over all irreducible components $\overline{\mathfrak{C}}$ of $\overline{\mathfrak{X}}^{w}$ and where im $(\overline{\mathfrak{C}})$ is the image of $\overline{\mathfrak{C}}$ in $\left(\overline{\mathfrak{X}^{\prime}}\right)^{w^{\prime}}$.

In order to use the projection formula (Proposition 3.25), we will need the following lemma.

Lemma 7.2. Let $X$ and $X^{\prime}$ be integral finite-type $K$-schemes, and let $f: X \rightarrow X^{\prime}$ be a generically finite dominant morphism of degree $\delta$. Let $\mathscr{U}^{\prime} \subset\left(X^{\prime}\right)^{\text {an }}$ be an analytic domain, and let $\mathscr{U}=$ $\left(f^{\text {an }}\right)^{-1}\left(\mathscr{U}^{\prime}\right)$. If $\left.f^{\text {an }}\right|_{\mathscr{U}}: \mathscr{U} \rightarrow \mathscr{U}^{\prime}$ is finite, then it has pure degree $\delta$ (Section 3.15).

Proof. By [Har77, Exercise II.3.7], there is a dense open subscheme $U^{\prime} \subset X^{\prime}$ such that $U:=$ $f^{-1}\left(U^{\prime}\right) \rightarrow U^{\prime}$ is finite. Shrinking $U^{\prime}$ if necessary, we assume that $U^{\prime}$ is smooth. By Proposition 3.24 the morphism $U^{\text {an }} \rightarrow\left(U^{\prime}\right)^{\text {an }}$ is pure of degree $\delta$. Let $\mathscr{U}$ and $\mathscr{U}^{\prime}$ be as in the statement of the lemma. By Proposition 3.23(ii), we may assume that $\mathscr{U}=\mathscr{M}(\mathcal{A})$ and $\mathscr{U}^{\prime}=\mathscr{M}\left(\mathcal{A}^{\prime}\right)$ are affinoid. By [Con99, Lemma A.1.2(2)], $\mathscr{U}$ and $\mathscr{U}^{\prime}$ are equidimensional of the same dimension as $X$ and $X^{\prime}$, respectively. Therefore $\mathscr{U}^{\prime} \cap\left(X^{\prime} \backslash U^{\prime}\right)$ an is nowhere dense in $\mathscr{U}^{\prime}$. If $\mathscr{V}^{\prime}=\mathscr{M}\left(\mathcal{B}^{\prime}\right)$ is any connected affinoid subdomain of $\mathscr{U}^{\prime} \cap\left(U^{\prime}\right)^{\text {an }}$, then $\mathcal{B}^{\prime}$ is a domain because $\mathscr{V}^{\prime}$ is smooth, and $\mathscr{V}:=\left(f^{\text {an }}\right)^{-1}\left(\mathscr{V}^{\prime}\right) \rightarrow \mathscr{V}^{\prime}$ has (pure) degree $\delta$ because $\mathscr{V}^{\prime} \subset\left(U^{\prime}\right)^{\text {an }}$. Since $\mathscr{U}^{\prime} \cap\left(X^{\prime} \backslash U^{\prime}\right)^{\text {an }}$ is 


\section{BAKer, S. PAyne And J. RabinofF}

nowhere dense in $\mathscr{U}^{\prime}$, we can choose $\mathscr{V}^{\prime} \operatorname{such}$ that $\operatorname{Spec}\left(\mathcal{B}^{\prime}\right) \rightarrow \operatorname{Spec}\left(\mathcal{A}^{\prime}\right)$ takes the generic point of $\operatorname{Spec}\left(\mathcal{B}^{\prime}\right)$ to any given generic point of $\operatorname{Spec}\left(\mathcal{A}^{\prime}\right)$. Hence $\mathscr{U} \rightarrow \mathscr{U}^{\prime}$ has pure degree $\delta$.

Proof of Theorem 7.1. Let $w^{\prime} \in \operatorname{Trop}\left(X^{\prime}\right) \cap N_{G}^{\prime}$ be a point with finite preimage under $F$. Let

$$
\mathscr{X}^{w^{\prime}}=\operatorname{trop}^{-1}\left(F^{-1}\left(w^{\prime}\right)\right) \cap X^{\text {an }}=\coprod_{w \in F^{-1}\left(w^{\prime}\right)} \mathscr{X}^{w} .
$$

This is an affinoid domain in $X^{\text {an }}$ because it is a closed subspace of the affinoid trop ${ }^{-1}\left(F^{-1}\left(w^{\prime}\right)\right)=$ $\coprod_{w \in F^{-1}\left(w^{\prime}\right)} \mathscr{U}^{w}$. We claim that $\mathscr{X}^{w^{\prime}} \rightarrow\left(\mathscr{X}^{\prime}\right)^{w^{\prime}}$ is a finite morphism. It suffices to show that the composite $\mathscr{X}^{w^{\prime}} \rightarrow \mathscr{U}^{w^{\prime}}$ is a finite morphism, where $\mathscr{U}^{w^{\prime}}=\operatorname{trop}^{-1}\left(w^{\prime}\right) \subset\left(\mathbf{T}^{\prime}\right)^{\text {an }}$. This follows exactly as in the proof of Theorem 4.30: Since $F^{-1}\left(w^{\prime}\right)$ is bounded, there is an affinoid domain of $\mathbf{T}^{\text {an }}$ contained in $\left(\alpha^{\text {an }}\right)^{-1}\left(\mathscr{U}^{w^{\prime}}\right)$ and containing $\mathscr{X}^{w^{\prime}}$ in its relative interior. This means that the morphism $\mathscr{X}^{w^{\prime}} \rightarrow\left(\mathscr{X}^{\prime}\right)^{w^{\prime}}$ is proper, thus finite because both spaces are affinoid. Hence by Lemma 7.2 the morphism $\mathscr{X}^{w^{\prime}} \rightarrow\left(\mathscr{X}^{\prime}\right)^{w^{\prime}}$ has pure degree $\delta$. Let $\mathfrak{X}^{w^{\prime}}:=\coprod_{w \in F^{-1}\left(w^{\prime}\right)} \mathfrak{X}^{w}$. The generic fiber of $\mathfrak{X}^{w^{\prime}}$ is $\mathscr{X}^{w^{\prime}}$, and the natural morphism $\mathfrak{X}^{w^{\prime}} \rightarrow\left(\mathfrak{X}^{\prime}\right)^{w^{\prime}}$ is finite by Proposition 3.8(ii) and takes generic points to generic points by Proposition 3.10. By Proposition 3.25 the induced morphism $\overline{\mathfrak{X}}^{w^{\prime}} \rightarrow\left(\overline{\mathfrak{X}}^{\prime}\right)^{w^{\prime}}$ has pure degree $\delta$, so summing (3.18.1) over all irreducible components $\overline{\mathfrak{C}^{\prime}}$ of $\left(\overline{\mathfrak{X}^{\prime}}\right)^{w^{\prime}}$ yields

$$
\delta \cdot m_{\operatorname{Trop}}\left(w^{\prime}\right)=\delta \sum_{\overline{\mathfrak{C}^{\prime}} \subset\left(\overline{\overline{\mathfrak{X}}^{\prime}}\right) w^{\prime}} \operatorname{mult}_{\left(\overline{\mathfrak{X}^{\prime}}\right) w^{\prime}}\left(\overline{\mathfrak{C}^{\prime}}\right)=\sum_{\overline{\mathfrak{C}} \subset \overline{\overline{\mathfrak{X}}^{\prime}}} \operatorname{mult}_{\overline{\mathfrak{X}^{\prime}}}\left(\overline{\mathfrak{C}^{\prime}}\right)[\overline{\mathfrak{C}}: \operatorname{im}(\overline{\mathfrak{C}})] .
$$

Since $\mathfrak{X}^{w^{\prime}}=\coprod_{w \in F^{-1}\left(w^{\prime}\right)} \mathfrak{X}^{w}$, this is the desired multiplicity formula.

As a consequence of Theorem 7.1, we obtain the following result.

Corollary 7.3. Let $\alpha: \mathbf{T} \rightarrow \mathbf{T}^{\prime}$ be a homomorphism of algebraic tori over $K$, and let $A=$ $\operatorname{trop}(\alpha): N_{\mathbb{R}} \rightarrow N_{\mathbb{R}}^{\prime}$ be the natural linear map. Let $X$ be a closed subvariety of $\mathbf{T}$, and suppose that $\alpha$ induces a generically finite morphism of degree $\delta$ from $X$ onto its schematic image $X^{\prime}$ in $\mathbf{T}^{\prime}$. After subdividing, we may assume that $A$ maps each face of $\operatorname{Trop}(X)$ onto a face of $\operatorname{Trop}\left(X^{\prime}\right)$. Let $\sigma^{\prime}$ be a maximal face of $\operatorname{Trop}\left(X^{\prime}\right)$. Then

$$
m\left(\sigma^{\prime}\right)=\frac{1}{\delta} \sum_{A(\sigma)=\sigma^{\prime}} m(\sigma) \cdot\left[N_{\sigma^{\prime}}^{\prime}: A\left(N_{\sigma}\right)\right] .
$$

(Here $N_{\sigma}$ and $N_{\sigma^{\prime}}^{\prime}$ are the sublattices of $N$ and $N^{\prime}$ parallel to $\sigma$ and $\sigma^{\prime}$, respectively.)

Proof. If $w^{\prime}$ is a smooth point of $\operatorname{Trop}\left(X^{\prime}\right)$ and $w$ is a smooth point of $\operatorname{Trop}(X)$ with $A(w)=w^{\prime}$, then $\left(\overline{\mathfrak{X}}^{\prime}\right)^{w^{\prime}} \cong Y^{\prime} \times T^{\prime}\left(w^{\prime}\right)$ and $\overline{\mathfrak{X}}^{w} \cong Y \times T(w)$, where $Y$ and $Y^{\prime}$ are 0 -dimensional schemes of length $m_{\text {Trop }}(w)$ and $m_{\text {Trop }}\left(w^{\prime}\right)$, respectively, and $T(w)$ and $T^{\prime}\left(w^{\prime}\right)$ are algebraic tori of dimension $\operatorname{dim}(X)=\operatorname{dim}\left(X^{\prime}\right)$ (cf. Remark 4.27). Moreover, $\alpha$ induces a finite homomorphism $T(w) \rightarrow$ $T^{\prime}\left(w^{\prime}\right)$ of degree $\left[N_{\sigma^{\prime}}^{\prime}: A\left(N_{\sigma}\right)\right]$. In this situation, the quantity $[\overline{\mathfrak{C}}: \operatorname{im}(\overline{\mathfrak{C}})]$ appearing in $(7.1 .1)$ is equal to $\left[T(w): T^{\prime}\left(w^{\prime}\right)\right]=\left[N_{\sigma^{\prime}}^{\prime}: A\left(N_{\sigma}\right)\right]$, and $m_{\text {Trop }}(w)=\sum_{\overline{\mathfrak{C}} \subset \overline{\mathfrak{X}} w}$ mult $\overline{\mathfrak{X}} w(\overline{\mathfrak{C}})$, so we are reduced to Theorem 7.1 .

Remark 7.4. The original Sturmfels-Tevelev multiplicity formula is the special case of Corollary 7.3 in which $K=k\{\{T\}\}$ and $X$ is defined over $k$. 


\section{NONARCHIMEDEAN GEOMETRY AND TROPICALIZATION}

\section{ACKNOWLEDGEMEnTS}

The authors would like to express their thanks to María Angélica Cueto, Eric Katz, Brian Osserman, David Speyer, and Josephine Yu for helpful discussions, and to Bernd Sturmfels for his interest and encouragement. Special thanks are due to Walter Gubler for some illuminating conversations which helped shape the direction of this work. The authors also thank Melanie Dunn for computing several nice examples of tropicalizations, and the anonymous referees for many helpful comments.

\section{REFERENCES}

ABBR15a O. Amini, M. Baker, E. Brugallé, and J. Rabinoff, Lifting harmonic morphisms I: Metrized complexes and Berkovich skeleta, Res. Math. Sci. 2 (2015), no. 7; http://dx.doi.org/10. 1186/s40687-014-0019-0.

ABBR15b , Lifting harmonic morphisms II: Tropical curves and metrized complexes, Algebra Number Theory 9 (2015), no. 2, 267-315; http://dx.doi.org/10.2140/ant.2015.9.267.

Bak93 H.F. Baker, Examples of applications of Newton's polygon to the theory of singular points of algebraic functions, Trans. Cambridge Philos. Soc. 15 (1893), 403-450.

Bak08 M. Baker, An introduction to Berkovich analytic spaces and non-Archimedean potential theory on curves, p-Adic Geometry, Univ. Lecture Ser., vol. 45 (Amer. Math. Soc., Providence, RI, 2008), 123-174; http://dx.doi.org/10.1090/ulect/045/04.

Bee09 P. Beelen, A generalization of Baker's theorem, Finite Fields Appl. 15 (2009), no. 5, 558-568; http://dx.doi.org/10.1016/j.ffa.2009.04.003.

Ber90 V. G. Berkovich, Spectral theory and analytic geometry over non-Archimedean fields, Math. Surveys and Monogr., vol. 33 (Amer. Math. Soc., Providence, RI, 1990).

Ber93 _ Étale cohomology for non-Archimedean analytic spaces, Publ. Math. Inst. Hautes Études Sci. (1993), no. 78, 5-161; http://dx.doi.org/10.1007/BF02712916.

Ber99 Smooth p-adic analytic spaces are locally contractible, Invent. Math. 137 (1999), no. 1, 1-84; http://dx.doi.org/10.1007/s002220050323.

Ber04_ Smooth p-adic analytic spaces are locally contractible II, in Geometric Aspects of Dwork Theory (Walter de Gruyter GmbH \& Co. KG, Berlin, 2004), 293-370.

BG84 R. Bieri and J.R. J. Groves, The geometry of the set of characters induced by valuations, J. reine angew. Math. 347 (1984), 168-195; http://dx.doi.org/10.1515/crll.1984.347. 168.

BGR84 S. Bosch, U. Güntzer, and R. Remmert, Non-Archimedean analysis. A systematic approach to rigid analytic geometry, Grundlehren Math. Wiss. vol. 261 (Springer-Verlag, Berlin, 1984); http://dx.doi.org/10.1007/978-3-642-52229-1.

BL85 S. Bosch and W. Lütkebohmert, Stable reduction and uniformization of abelian varieties I, Math. Ann. 270 (1985), no. 3, 349-379; http://dx.doi.org/10.1007/BF01473432.

BL93 _ Formal and rigid geometry I: Rigid spaces, Math. Ann. 295 (1993), no. 2, 291-317; http://dx.doi.org/10.1007/BF01444889.

Bos14 S. Bosch, Lectures on formal and rigid geometry, Lecture Notes in Math., vol. 2105 (Springer, Cham, 2014); http://dx.doi.org/10.1007/978-3-319-04417-0.

BPR13 M. Baker, S. Payne, and J. Rabinoff, On the structure of non-Archimedean analytic curves, in Tropical and non-Archimedean geometry, Contemp. Math. vol. 605, Amer. Math. Soc. (Providence, RI, 2013), 93-121; http://dx.doi.org/10.1090/conm/605/12113.

BR10 M. Baker and R. Rumely, Potential theory and dynamics on the Berkovich projective line, Math. Surveys and Monogr. vol. 159 (Amer. Math. Soc., Providence, RI, 2010); http://dx. doi.org/10.1090/surv/159. 


\section{BAKer, S. PAyne And J. RABinofF}

CHW14 M.A. Cueto, M. Häbich, and A. Werner, Faithful tropicalization of the Grassmannian of planes, Math. Ann. 360 (2014), no. 1-2, 391-437; http://dx.doi.org/10.1007/ s00208-014-1037-3.

Con99 B. Conrad, Irreducible components of rigid spaces, Ann. Inst. Fourier (Grenoble) 49 (1999), no. 2, 473-541; http://dx.doi.org/10.5802/aif.1681.

Con08_ Several approaches to non-Archimedean geometry, in p-Adic Geometry, Univ. Lecture Ser., vol. 45, Amer. Math. Soc. (Providence, RI, 2008), 9-63; http://dx.doi.org/10.1090/ ulect/045/02.

CS13 M. Chan and B. Sturmfels, Elliptic curves in honeycomb form, in Algebraic and Combinatorial Aspects of Tropical Geometry, Contemp. Math., vol. 589 (Amer. Math. Soc., Providence, RI, 2013), 87-107; http://dx.doi.org/10.1090/conm/589/11743.

DP14 J. Draisma and E. Postinghel, Faithful tropicalisation and torus actions, arXiv:1404.4715.

Duc09 A. Ducros, Les espaces de Berkovich sont excellents, Ann. Inst. Fourier (Grenoble) 59 (2009), no. 4, 1443-1552; http://dx.doi.org/10.5802/aif.2470.

EKL06 M. Einsiedler, M. Kapranov, and D. Lind, Non-Archimedean amoebas and tropical varieties, J. Reine Angew. Math. 601 (2006), 139-157; http://dx.doi.org/10.1515/CRELLE. 2006. 097.

FM86 J. Fresnel and M. Matignon, Sur les espaces analytiques quasi-compacts de dimension 1 sur un corps valué complet ultramétrique, Ann. Mat. Pura Appl. (4) 145 (1986), 159-210; http: //dx.doi.org/10.1007/BF01790541.

Ful93 W. Fulton, Introduction to toric varieties, Ann. of Math. Stud., vol. 131 (Princeton University Press, Princeton, NJ, 1993).

GD66 A. Grothendieck and J. Dieudonné, Éléments de géométrie algébrique. IV. Étude locale des schémas et des morphismes de schémas. III, Publ. Math. Inst. Hautes Études Sci. (1966), no. 28, 5-248; http://dx.doi.org/10.1007/BF02684343, http://www.numdam.org/item? id=PMIHES_1966__28__5_0.

GRW14 W. Gubler, J. Rabinoff, and A. Werner, Skeletons and tropicalizations, arXiv:1404.7044.

Gub98 W. Gubler, Local heights of subvarieties over non-Archimedean fields, J. reine angew. Math. 498 (1998), 61-113; http://dx.doi.org/10.1515/crll.1998.054.

Gub07a_ The Bogomolov conjecture for totally degenerate abelian varieties, Invent. Math. 169 (2007), no. 2, 377-400; http://dx.doi.org/10.1007/s00222-007-0049-y.

Gub07b_L Tropical varieties for non-Archimedean analytic spaces, Invent. Math. 169 (2007), no. 2, 321-376; http://dx.doi.org/10.1007/s00222-007-0048-z.

Gub13 - A guide to tropicalizations, in Algebraic and Combinatorial Aspects of Tropical Geometry, Contemp. Math., vol. 589 (Amer. Math. Soc., Providence, RI, 2013), 125-189; http: //dx.doi.org/10.1090/conm/589/11745.

Har77 R. Hartshorne, Algebraic geometry, Grad. Texts in Math., vol. 52 (Springer-Verlag, New York Heidelberg, 1977); http://dx.doi.org/10.1007/978-1-4757-3849-0.

HK12 D. Helm and E. Katz, Monodromy filtrations and the topology of tropical varieties, Canad. J. Math. 64 (2012), no. 4, 845-868; http://dx.doi.org/10.4153/CJM-2011-067-9.

Kat12 E. Katz, Lifting tropical curves in space and linear systems on graphs, Adv. Math. 230 (2012), no. 3, 853-875; http://dx.doi.org/10.1016/j.aim.2012.03.017.

KMM08 E. Katz, H. Markwig, and T. Markwig, The $j$-invariant of a plane tropical cubic, J. Algebra 320 (2008), no. 10, 3832-3848; http://dx.doi.org/10.1016/j.jalgebra.2008.08.018.

KMM09 , The tropical j-invariant, LMS J. Comput. Math. 12 (2009), 275-294; http://dx. doi.org/10.1112/S1461157000001522.

MFK94 D. Mumford, J. Fogarty, and F. Kirwan, Geometric invariant theory, third ed., Ergeb. Math. Grenzgeb. (2), vol. 34 (Springer-Verlag, Berlin, 1994); http://dx.doi.org/10.1007/ 978-3-642-57916-5. 


\section{NONARCHIMEDEAN GEOMETRY AND TROPICALIZATION}

MS15 D. Maclagan and B. Sturmfels, Introduction to tropical geometry, Grad. Stud. Math., vol. 161 (Amer. Math. Soc., Providence, RI, 2015).

Nis09 T. Nishinou, Correspondence theorems for tropical curves, arXiv:0912.5090.

OP13 B. Osserman and S. Payne, Lifting tropical intersections, Doc. Math. 18 (2013), 121-175.

Pap05 A. Papadopoulos, Metric spaces, convexity and nonpositive curvature, IRMA Lect. Math. Theor. Phys., vol. 6, (European Mathematical Society, Zürich, 2005); http://dx.doi.org/ 10.4171/132.

Pay09a S. Payne, Analytification is the limit of all tropicalizations, Math. Res. Lett. 16 (2009), no. 3, 543-556; http://dx.doi.org/10.4310/MRL.2009.v16.n3.a13.

Pay09b_, Fibers of tropicalization, Math. Z. 262 (2009), no. 2, 301-311; http://dx.doi.org/ 10.1007/s00209-008-0374-x.

Pay12 , Erratum to "Fibers of tropicalization", Math. Z. 272 (2012), no. 3-4, 1403-1406; http://dx.doi.org/10.1007/s00209-012-1080-2.

Rab12 J. Rabinoff, Tropical analytic geometry, Newton polygons, and tropical intersections, Adv. Math. 229 (2012), no. 6, 3192-3255; http://dx.doi.org/10.1016/j.aim.2012.02.003.

RG71 M. Raynaud and L. Gruson, Critères de platitude et de projectivité. Techniques de "platification" d'un module, Invent. Math. 13 (1971), 1-89; http://dx. doi .org/10.1007/BF01390094.

Rob00 A. M. Robert, A course in p-adic analysis, Grad. Texts in Math., vol. 198 (Springer-Verlag, New York, 2000); http://dx.doi.org/10.1007/978-1-4757-3254-2.

Sil09 J.H. Silverman, The arithmetic of elliptic curves, second ed., Grad. Texts in Math., vol. 106 (Springer, Dordrecht, 2009), http://dx.doi.org/10.1007/978-0-387-09494-6.

Spe05 D. E. Speyer, Tropical geometry, Ph.D. thesis, University of California, Berkeley, 2005, available at http://www-personal.umich.edu/ speyer/thesis.pdf.

Spe14 , Parameterizing tropical curves I: Curves of genus zero and one, Algebra Number Theory 8 (2014), no. 4, 963-998; http://dx.doi.org/10.2140/ant.2014.8.963.

ST08 B. Sturmfels and J. Tevelev, Elimination theory for tropical varieties, Math. Res. Lett. 15 (2008), no. 3, 543-562; http://dx.doi.org/10.4310/MRL.2008.v15.n3.a14.

Tev07 J. Tevelev, Compactifications of subvarieties of tori, Amer. J. Math. 129 (2007), no. 4, 10871104; http://dx.doi.org/10.1353/ajm.2007.0029.

Tho90 A. Thorup, Rational equivalence theory on arbitrary Noetherian schemes, Enumerative Geometry (Sitges, 1987), Lecture Notes in Math., vol. 1436 (Springer, Berlin, 1990), 256-297; http://dx.doi.org/10.1007/BFb0084049.

Thu05 A. Thuillier, Théorie du potentiel sur les courbes en géométrie analytique non archimédienne. Applications à la théorie d'Arakelov, Ph.D. thesis, University of Rennes, 2005, available at http://tel.ccsd.cnrs.fr/documents/archives0/00/01/09/90/index.html.

Vaq00 M. Vaquié, Valuations, Resolution of Singularities (Obergurgl, 1997), Progr. Math., vol. 181 (Birkhäuser, Basel, 2000), 539-590; http://dx.doi.org/10.1007/978-3-0348-8399-3_21.

Matthew Baker mbaker@math.gatech.edu

School of Mathematics, Georgia Institute of Technology, Atlanta GA 30332-0160, USA

Sam Payne sam.payne@yale.edu

Mathematics Department, Yale University, New Haven, CT 06511, USA

Joseph Rabinoff rabinoff@math.gatech.edu

Department of Mathematics, Harvard University, Cambridge, MA 02138, USA

Current address: School of Mathematics, Georgia Institute of Technology, Atlanta GA 303320160, USA 